\title{
Neutrino Factories: Realization and Physics Potential
}

\author{
S. Geer* and M. S. Zisman ${ }^{\dagger}$
}

December 5, 2006

\begin{abstract}
Neutrino Factories offer an exciting option for the long-term neutrino physics program. This new type of neutrino facility will provide beams with unique properties. Low systematic uncertainties at a Neutrino Factory, together with a unique and precisely known neutrino flavor content, will enable neutrino oscillation measurements to be made with unprecedented sensitivity and precision. Over recent years, the resulting neutrino factory physics potential has been discussed extensively in the literature. In addition, over the last six years the R\&D necessary to realize a Neutrino Factory has been progressing, and has developed into a significant international activity. It is expected that, within about five more years, the initial phase of this R\&D program will be complete and, if the community chooses to build this new type of neutrino source within the following decade, neutrino factory technology will be ready for the final $\mathrm{R} \& \mathrm{D}$ phase prior to construction. In this paper $(i)$ an overview is given of the technical ingredients needed for a Neutrino Factory, $(i i)$ beam properties are described, (iii) the resulting neutrino oscillation physics potential is summarized, $(i v)$ a more detailed description is given for one representative Neutrino Factory design, and $(v)$ the ongoing $R \& D$ program is summarized, and future plans briefly described.
\end{abstract}

\section{Introduction}

New accelerator technologies offer the possibility of building, in the not-too-distant future, an accelerator complex to produce and capture more than $10^{20}$ muons per year [1]. It has been proposed to build a Neutrino Factory (NF) [2; 3] by accelerating the muons from this intense source to energies of several tens of $\mathrm{GeV}$, injecting them into a storage ring having long straight sections, and exploiting the intense neutrino beams that are produced by muons decaying in the straight sections. The decays

$$
\mu^{-} \rightarrow e^{-} \nu_{\mu} \bar{\nu}_{e}, \quad \mu^{+} \rightarrow e^{+} \bar{\nu}_{\mu} \nu_{e}
$$

offer the exciting possibility of studying neutrino oscillations and neutrino interactions with exquisite precision. If pointing in a suitable direction, a NF beam would be sufficiently intense to produce a detectable event rate on the other side of the Earth. With shorter baselines of a few thousand kilometers or less, very large data samples could be recorded, enabling neutrino oscillations to be studied with high statistical precision and, since the neutrino beam properties would be very well known, low systematic uncertainties. In addition, the presence of electron-type neutrinos (anti-neutrinos) together with muontype anti-neutrinos (neutrinos) in the beam makes NF beams qualitatively different from conventional

${ }^{*}$ Fermi National Accelerator Laboratory, P.O. Box 500, Batavia, Illinois, U.S.A.

${ }^{\dagger}$ Lawrence Berkeley National Laboratory, One Cyclotron Road, Berkeley, CA 94720, U.S.A. 
muon-type neutrino and anti-neutrino beams produced from charged pion decays. The electron-type neutrinos and anti-neutrinos would enable extremely sensitive measurements of $\nu_{e} \rightarrow \nu_{\mu}, \bar{\nu}_{e} \rightarrow \bar{\nu}_{\mu}$, $\nu_{e} \rightarrow \nu_{\tau}$, and $\bar{\nu}_{e} \rightarrow \bar{\nu}_{\tau}$ transitions with very low background rates.

Neutrino Factories require very intense beams of muons with energies of a few tens of GeV. The design ideas for producing a very intense high energy muon beam have been developing over the last few decades. Muons are created in the decays of charged pions. A high intensity muon source requires a high intensity charged-pion source, which in turn requires a high intensity primary beam, a pion production target, and an efficient pion collection scheme. An efficient collection scheme based on a target within a high-field solenoid was proposed by Dijikibaev and Lobashev in 1989 [4], and subsequently developed by Palmer et al. [5]. If the solenoidal field is sufficiently high, essentially all of the charged pions produced in multi-GeV proton interactions can be captured radially, maximizing the number captured within a lower-field larger-aperture solenoid decay channel. The majority of the pions have momenta of a few hundred $\mathrm{MeV} / \mathrm{c}$ with a large momentum spread, and transverse momentum components that are comparable to their longitudinal momenta. Hence, when they decay, the pions produce daughter muons that occupy a very large longitudinal and transverse phase space. The transverse phase space and the muon momentum spread are much larger than the phase space that can be accepted by a normal type of accelerator. Hence, before acceleration, it is necessary to manipulate the phase space occupied by the muons. The momentum spread can be reduced by allowing an energy-time correlation to develop in a long drift space, and then using a time-dependent acceleration system to accelerate the late (slower) particles and decelerate the early (fast) particles ("phase rotation"). The muons can then be captured within bunches using a string of rf cavities. At this point the muons are packaged and captured in bunches and confined radially within a magnetic channel. However, they still occupy a very large transverse phase space. Two different concepts have been proposed to accelerate the muon bunches. The first concept is to cool the muon bunches in transverse phase space using "ionization cooling." This new cooling technique was proposed by Kolomensky in 1965 [6], and the concept was subsequently developed by Skrinsky and Parkhomchuk [7], Neuffer [8; 9], and others. After ionization cooling, the transverse phase space has been reduced such that the resulting beam fits within the acceptance of a normal type of accelerator. The alternative concept, proposed in 1999 by Mori et al., is to use a system of Fixed Field Alternating Gradient (FFAG) accelerators, which have an exceptionally large acceptance.

By the end of the 1980s, all of the basic concepts needed to design a multi-GeV very intense muon source were in place. However, a very significant effort was required to go beyond the initial concepts and develop the required technology together with a practical scheme. The inital motivation for this effort emerged in the Sausalito Workshop [10] in 1994, where it was realized that, using the solenoid pion collection and muon ionization cooling ideas, a high energy Muon Collider (MC) might be feasible. To explore the feasibility of designing a practical MC, an informal collaboration of about 100 scientists and engineers was formed in the U.S., and produced an initial "Muon Collider Feasibility Study" report in 1996 [1]. The initial results from this work were encouraging. The U.S. Muon Collider Collaboration became a formal entity in 1997, and received its first significant funding in Spring 1998. Meanwhile, in November 1997, the NF concept was proposed [2] in a "Workshop on the Physics at the First Muon Collider and at the Front-End of a Muon Collider" [12. It was demonstrated that a MC-class muon source plus a suitable acceleration scheme would, if used to fill a storage ring with long straight sections, produce a high-intensity neutrino beam well suited for future precision neutrino oscillation studies. In 1998, the physics case was taken a step further when De Rujula, Gavela, and Hernandez [13] showed that a NF experiment might measure leptonic CP-Violation, and Barger, Geer, and Whisnant [14] showed that, with a NF, matter effects [15; 16] could be exploited to determine the neutrino mass hierarchy.

Motivated by these developments, together with the exciting atmospheric neutrino oscillation discovery by the Super-Kamiokande Experiment [17] in Japan, in 1999 the Muon Collider Collaboration became the Neutrino Factory and Muon Collider Collaboration (NFMCC [18]). The NFMCC embarked on a series of NF design studies, each building on the past and working towards a practical, 
high-performance, cost-effective design. Study I [19] was sponsored and organized by Fermilab, and fully involved the NFMCC together with additional expertise from the U.S., Europe, and Japan. Study I, which was completed in 2001, established the feasibility of building a NF and defined the hardware R\&D required before this new type of accelerator facility could be built. However, the initial design did not emphasize cost-effectiveness, and simulations of its performance failed, by a factor of a few, to meet the muon intensity goal set by the physics study [20] conducted in parallel with Study I. The lessons learned during Study I motivated a second NF study to produce a design with improved performance. Study II [21] was sponsored by BNL and organized by BNL together with the NFMCC. In 2002, this second study resulted in an improved design that maintained feasibility while achieving a simulated performance that met the design goal. Once again, no emphasis was placed on cost effectiveness. Over the next few years, further work within the NFMCC resulted in more cost-effective ideas for the design of the key NF subsystems. In 2004, the baseline NFMCC design was updated (Study IIa [22; 23]) to incorporate these ideas. This resulted in a simpler design with an improved performance and a cost estimate that was $2 / 3$ of the corresponding estimate for Study II. Note that Studies I and II included sufficient engineering support to produce defensible cost estimates for the respective NF designs. Study IIa had no engineering support, but benefited from the Study II cost estimate, which enabled costs for the Study IIa design to be scaled from the corresponding estimates for Study II.

During the period in which Studies I and II were being conducted in the U.S., NF concepts were also being developed in Europe [24; 25] and Japan [26]. The "Prospective Study of Muon Storage Rings at CERN" was completed in 1999 [27], and led to the development at CERN of a NF design similar to the U.S. concept, but with different technical solutions for the various subsystems. The CERN design also differed by using a bow-tie shaped storage ring instead of a racetrack geometry. This enabled the two long straight sections to each produce a useful neutrino beam pointing at a different distant detector. In 2001, a "Feasibility Study of a Neutrino Factory in Japan" produced a report [26] that proposed a third, and very different, NF concept in which there is no ionization cooling. Instead, a series of FFAG accelerators is used to accept muon bunches occupying a very large longitudinal and transverse phase space, and accelerate them to high energy [28].

All of the NF studies have concluded that a Neutrino Factory based on a muon storage ring is feasible, although a vigorous R\&D activity is needed to develop the required technology. The 2001 HEPAP subpanel 29 recommended a level of support sufficient to perform the critical accelerator R\&D within 10-15 years. This R\&D effort is ongoing and, with encouragment from the neutrino community, has grown into a significant international enterprise that includes hardware component development, NF design studies, and two international subsystem demonstration experiments. This R\&D activity has spawned, and is continuing to spawn, new ideas, new inventions, and new understanding.

In this paper $(i)$ an overview is given of the technical ingredients needed for a NF, $(i i)$ beam properties are described, $(i i i)$ the resulting neutrino oscillation physics potential is summarized, $(i v)$ a more detailed description is given for one representativeNF design, and $(v)$ the ongoing $\mathrm{R} \& \mathrm{D}$ program is summarized, and future plans briefly described.

\section{Machine Overview}

In this Section, we briefly describe the basic concepts that are used to design a NF. The primary beam is a high intensity proton beam of typical energy $2-50 \mathrm{GeV}$. The proton beam impinges on a target, typically a high- $Z$ material, and interacts to produce a secondary charged pion beam. Within a few tens of meters the pions decay to produce muons, creating a longer-lived tertiary muon beam. The remainder of the NF complex serves three functions:

- to condition the muon beam 
- to accelerate it rapidly to its desired final energy (generally a few tens of GeV)

- to store it in a decay ring having one or more long straight sections

Muons decaying in the straight sections create neutrino beams pointing in the direction of the straight section, which can be oriented towards a detector located thousands of kilometers from the source.

Two different approaches to the design of a NF have been studied over the past several years. The first type is based on a "linear" front-end configuration (Fig. 1) and the second type on a ringbased configuration (Fig. 2). The linear configuration has been studied mainly in the U.S. [19; 21] and Europe [30], while the ring design-based on so-called "scaling" Fixed-Field, Alternating Gradient (FFAG) rings - has been examined in Japan [31].

Throughout this paper, we will discuss two distinct types of FFAG rings. The first type is referred to as a scaling FFAG, where the betatron tune stays fixed as the beam is accelerated. This is the traditional type of FFAG implementation, which makes use of very large aperture magnets. The second type, with a betatron tune that varies with energy, is referred to as a non-scaling FFAG. The magnets tend to be smaller for the non-scaling FFAG, but with the consequence that the beam dynamics is more complicated.

The main functional ingredients of a NF include a Proton Driver, a Target and Capture section, a Bunching and Phase Rotation section, an Ionization Cooling section, an Acceleration section, and, finally, a Decay Ring. The Proton Driver provides 1-4 MW of protons on target; both synchrotrons and linacs have been considered, and the possibility of an FFAG ring has also been contemplated. The Target and Capture section comprises a high power target immersed in a high-field $(\approx 20 \mathrm{~T})$ solenoid. In this configuration [21], the field is smoothly tapered down to a more modest value, say a few Tesla, over a length of about $10 \mathrm{~m}$. An alternative approach calls for a "horn" to collect the pions and focus them into the decay channel. Because the downstream portions of recent NF designs permit the simultaneous transmission of both signs of muon, however, the horn approach, due to its charge selectivity, is now generally disfavored.

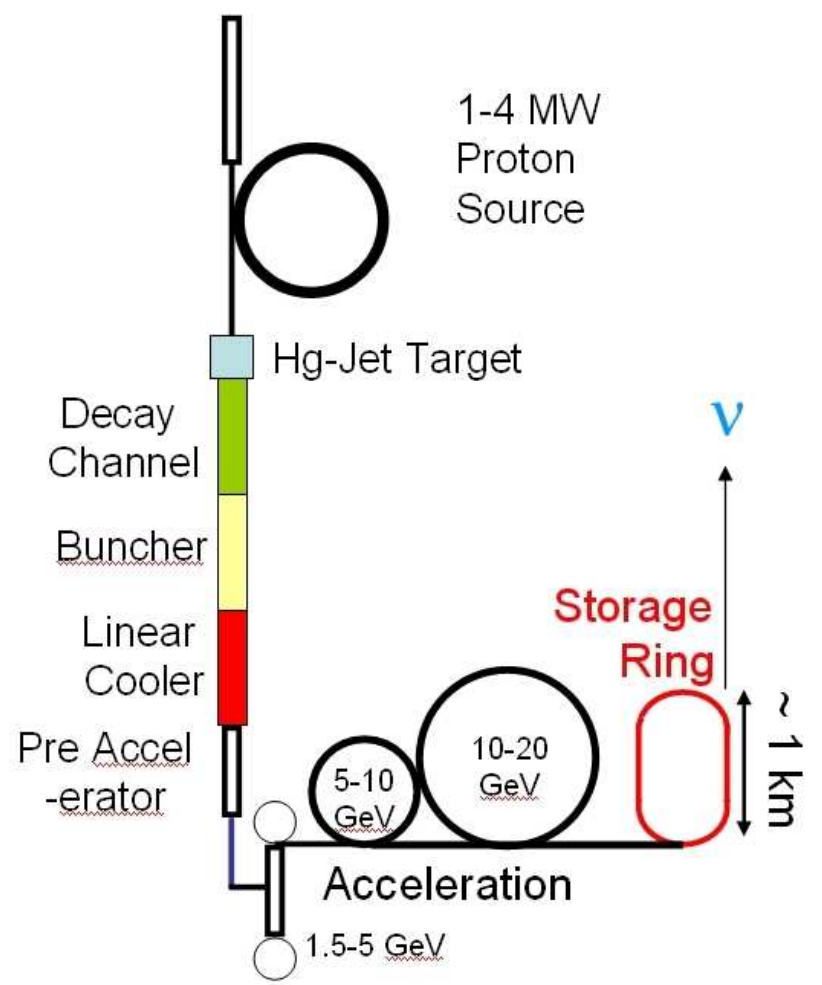

Figure 1: (Color) Neutrino Factory schematic (linear configuration). 


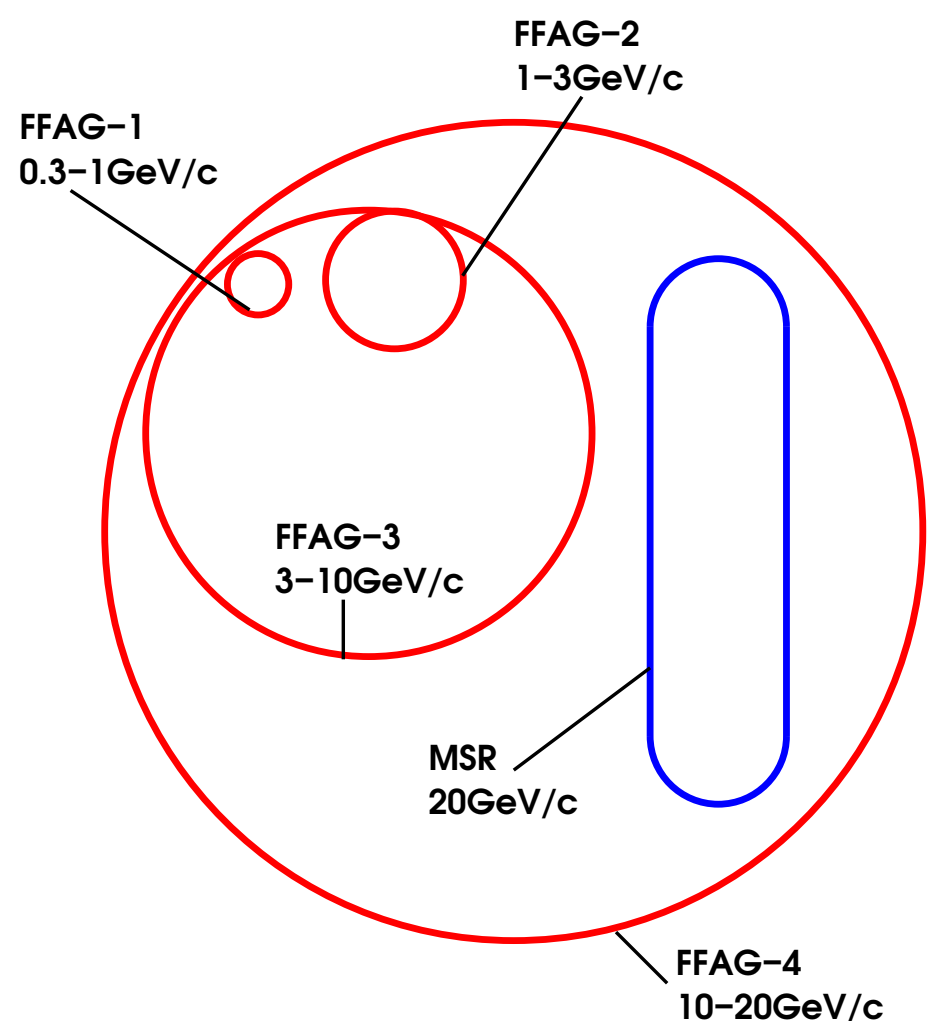

Figure 2: (Color) Neutrino Factory schematic (circular configuration).

For the linear front end, bunching is accomplished with rf cavities of modest gradient whose frequencies change along the beam line. After bunching the beam, another set of rf cavities is used to rotate the beam in longitudinal phase space to reduce its energy spread. The phase rotation cavities have higher gradients than those in the bunching region, and also having decreasing frequencies along the beam line. In the case of the circular front end, a very low frequency rf system (typically a few $\mathrm{MHz}$ ) is employed in an FFAG ring, permitting the capture of a bunch within a single rf bucket. This eliminates the need either to create a bunch train or to reduce the momentum spread of the bunches prior to rf capture.

In the linear front end, a solenoidal cooling channel, with high-gradient rf cavities (both 88- and 201-MHz systems have been considered) and energy absorbers, reduces the transverse normalized rms emittance of the muon beam by a factor of $2-10$. This "beam cooling" takes place at a central muon momentum of a few hundred $\mathrm{MeV} / \mathrm{c}$. To minimize the effects of multiple scattering, which "heats" the beam, a low- $Z$ absorber material is employed. Typical choices for absorber material have been liquid-hydrogen or LiH. For the circular front end, an uncooled beam is transported, which is possible because of the large transverse and longitudinal acceptance of an FFAG ring utilizing large aperture magnets and low-frequency rf.

Following the linear front end is a "pre-acceleration" section that makes use of a superconducting linac with solenoidal focusing, and is designed to raise the muon beam energy to $\approx 1 \mathrm{GeV}$. The preaccelerator is followed by either Recirculating Linear Accelerators (RLAs) or cascaded FFAG rings having quadrupole focusing, to reach, for example, $20 \mathrm{GeV}$. Additional RLA or FFAG stages would be added to reach a higher beam energy, if required. While, in practice, the details of the acceleration scheme tend to be project specific, some combination of these subsystems has been used in all cases presently studied. The circular front end already makes use of FFAG rings to capture the muon beam, and these are simply further cascaded to reach the required final energy. The typical energy gain with an FFAG is a factor of 2-3 so, in the circular scheme, four FFAG rings are needed to reach $20 \mathrm{GeV}$ and a fifth one would be needed to reach $50 \mathrm{GeV}$. 
A racetrack-, bow-tie-, or triangular-shaped superconducting storage ring is employed with either the linear or the circular front end. With a racetrack shape, $\approx 35 \%$ of the stored muons decay in the straight section pointed toward a detector located thousands of kilometers from the ring. With the triangular or bow-tie shaped ring, there are two straight sections within each of which $\approx 25 \%$ of the muons decay. The straight sections can be pointed in different directions towards detectors at different distances (baselines). In all these designs, muons survive for roughly 500 turns.

\subsection{Linear Configuration}

\subsubsection{Proton Driver}

The particular proton drivers that have been considered in detailed studies to date are: $(i)$ an upgraded synchrotron source at Fermilab (Study I); (ii) a $2 \mathrm{GeV}$ linac based proton source at CERN (CERN Study); (iii) an upgraded proton source at BNL (Study II and IIa); and (iv) the 50 GeV synchrotron under construction at J-PARC. Since Study IIa is the most recent of these studies, in the following we will take as representative an upgrade of the BNL Alternating Gradient Synchrotron (AGS). Parameters are listed in Table 1 .

To serve as the proton driver for a NF, the existing AGS booster would be replaced by a $1.2 \mathrm{GeV}$ superconducting proton linac. The modified layout is shown in Fig. 3. The AGS repetition rate would be increased from $0.5 \mathrm{~Hz}$ to $2.5 \mathrm{~Hz}$ by adding power supplies to permit ramping the ring more quickly. No new technology is required for this - the existing supplies would be replicated and the magnet strings would be split into six sectors rather than the two used presently. The total proton charge $\left(10^{14} \mathrm{ppp}\right.$ in six bunches) is only $40 \%$ higher than the current performance of the AGS. However, the bunches required for a NF are shorter than those presently used in the AGS, so there is a large increase in peak current and concomitant need for an improved vacuum chamber; this would be included in the upgrade. The six proton bunches are extracted separately, spaced by $20 \mathrm{~ms}$, so that the target and rf systems that follow need only deal with single pulses at an instantaneous repetition rate of $50 \mathrm{~Hz}$ (average rate of $15 \mathrm{~Hz}$ ). The average proton beam power is $1 \mathrm{MW}$. A possible future upgrade to $2 \times 10^{14} \mathrm{ppp}$ and $5 \mathrm{~Hz}$ could give an average beam power of $4 \mathrm{MW}$. At this higher intensity, a superconducting bunch compressor ring would be needed to maintain the rms bunch length at or below 3 ns.

If the facility were built at Fermilab, the proton driver would be newly constructed. A number of technical options are presently being explored [32; 33]. The second option, based on an 8-GeV superconducting linac, is potentially attractive in that it provides synergy with the International Linear

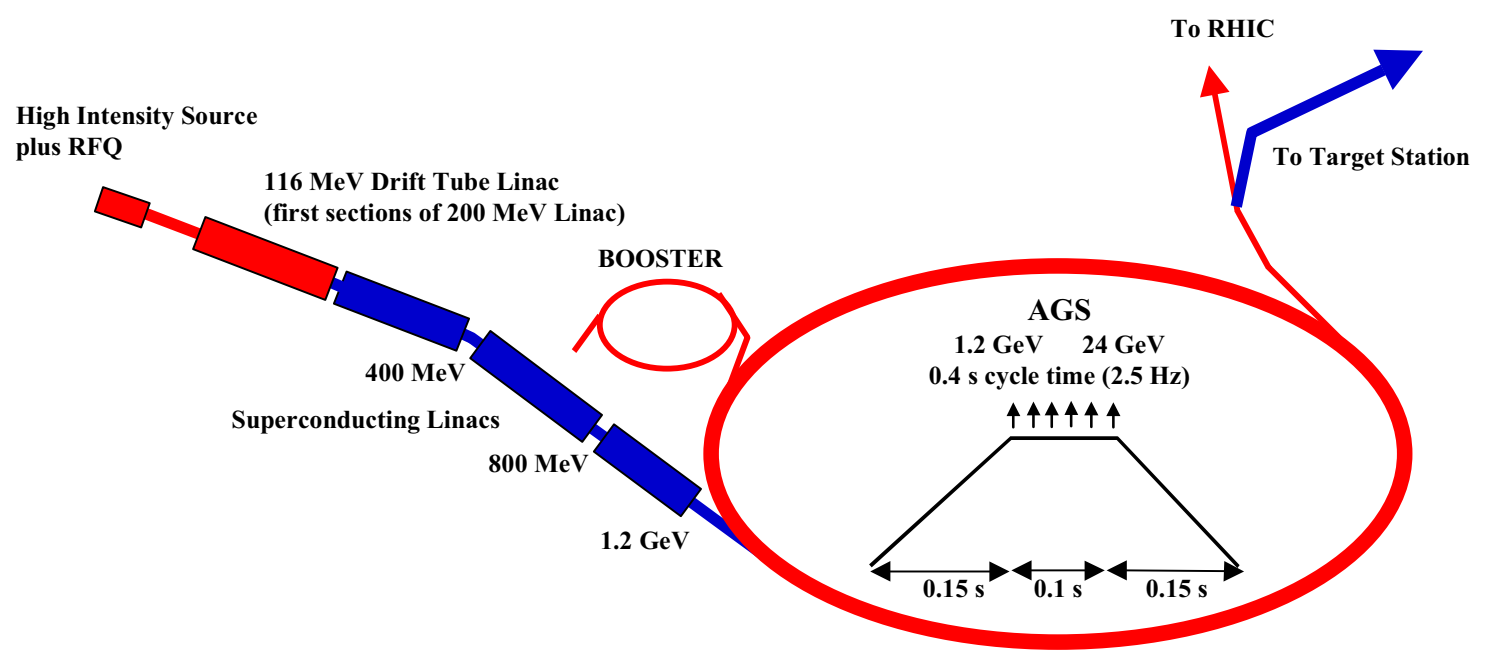

Figure 3: (Color) Representative proton driver layout. 
Collider (ILC) design effort. A CERN facility, or a facility at the Rutherford Appleton Laboratory (RAL) in the UK, might use either a linac-based or synchrotron-based upgraded proton source. A facility in Japan would likely use an upgraded source based on the $50 \mathrm{GeV}$ synchrotron at J-PARC.

\subsubsection{Target and Capture}

Various potential primary proton beam target concepts have been considered. The most developed of these is a mercury-jet target, chosen to give a high yield of pions per MW of incident proton power. The 1-cm-diameter jet is continuous, and is tilted with respect to the beam axis. The target layout proposed in Study II is shown in Fig. 4. The thermal shock from the interacting proton bunch is assumed to fully disperse the mercury, so the jet must have a velocity of $20-30 \mathrm{~m} / \mathrm{s}$ to allow the target material to be renewed before the next proton bunch arrives. Calculations of pion yields that reflect the detailed magnetic geometry of the target area have been performed with the MARS code [34] and are reported in Section 5. To avoid mechanical fatigue problems, a mercury pool serves as the beam dump. This pool is part of the overall target system-its mercury is circulated through the mercury-jet nozzle after passing through a heat exchanger.

Pions emerging from the target are captured and focused down the decay channel by a solenoidal field that is $20 \mathrm{~T}$ at the target center, and tapers down, over $12 \mathrm{~m}$, to $1.75 \mathrm{~T}$. (The Study II capture channel design was updated for Study IIa $22 ; 23$ to improve muon throughput.) The $20 \mathrm{~T}$ solenoid, with a resistive magnet insert and superconducting outer coil, is similar in character to higher field (up to $45 \mathrm{~T}$ ), but smaller bore, magnets existing at several laboratories [35]. The magnet insert is made with hollow copper conductor having ceramic insulation to withstand radiation. MARS simulations [36] of radiation levels show that, with the shielding provided, both the copper and superconducting magnets will have reasonable lifetime.

Alternative targets have also been considered, such as tungsten or tantalum rods [31]. The materials issues are severe for a solid target, so considerable $R \& D$ will be needed to validate this approach.

\subsubsection{Buncher and Phase Rotation}

Pions, and the muons into which they decay, are generated in the target over a very wide range of energies. To prepare the muon beam for acceleration thus requires significant "conditioning." In a typical scheme [22], the bunch is first drifted to develop a time-energy correlation, with higher energy particles at the head of the bunch and lower energy particles at the tail. Next, the long bunch is separated into a number of shorter bunches suitable for capture and acceleration in a high-frequency (e.g., 201-MHz) rf system. This is done with a series of rf cavities having frequencies that decrease along the beam line, separated by suitably chosen drift spaces. The resultant bunch train still has

Table 1: Proton driver parameters for Study II design.

\begin{tabular}{lc}
\hline Total beam power $(\mathrm{MW})$ & 1 \\
Beam energy $(\mathrm{GeV})$ & 24 \\
Average beam current $(\mu \mathrm{A})$ & 42 \\
Cycle time $(\mathrm{ms})$ & 400 \\
Number of protons per fill & $1 \times 10^{14}$ \\
Average circulating current $(\mathrm{A})$ & 6 \\
No. of bunches per fill & 6 \\
No. of protons per bunch & $1.7 \times 10^{13}$ \\
Time between extracted bunches $(\mathrm{ms})$ & 20 \\
Bunch length at extraction, rms $(\mathrm{ns})$ & 3 \\
\hline
\end{tabular}




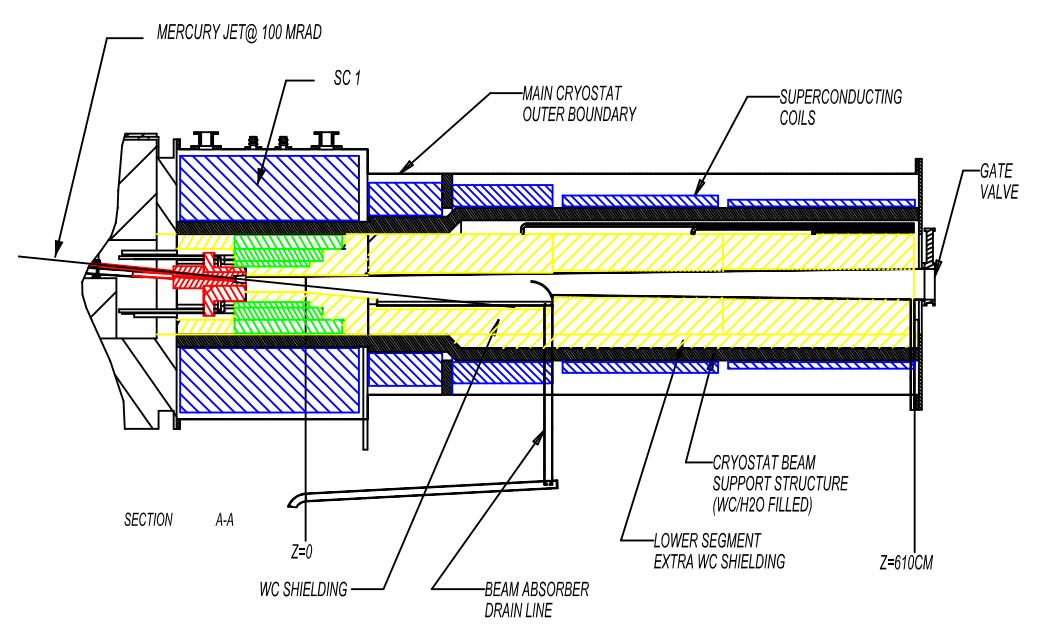

Figure 4: (Color) Target, capture solenoids and mercury containment.

a substantial energy correlation, with the higher energy bunches first and progressively lower energy bunches coming behind. The large energy tilt is then "phase rotated," using additional rf cavities and drifts, into a bunch train with a longer time duration and a lower energy spread. The beam at the end of the buncher and phase rotation section has an average momentum of about $220 \mathrm{MeV} / \mathrm{c}$. Note that the buncher and phase rotation system can transport both signs of muons in interleaved bunches.

\subsubsection{Cooling}

A new fast technique has been proposed, ionization cooling, in which the muons lose longitudinal and transverse momentum by ionization losses as they pass through an absorber. The longitudinal momentum is then replaced by reacceleration in $\mathrm{rf}$ cavities, and the process repeated many times. The emittance increase from Coulomb scattering is controlled by choosing low- $Z$ absorbers (e.g., liquid hydrogen or $\mathrm{LiH}$ ) and by maintaining the focusing strength such that the angular spread of the beam at the absorber locations is reasonably large compared with the rms multiple-scattering angle. In the Study IIa cooling lattice proposed in Refs. 222; 23], the energy absorbers are attached directly to the apertures of the rf cavity, thus serving the dual purposes of closing the cavity apertures electromagnetically (increasing the cavity shunt impedance, which decreases the peak power required to achieve a given accelerating gradient) and providing energy loss. Compared with the approach used in Study II, the Study IIa absorbers are more distributed, and so do not lend themselves to being located at an optical focus. Therefore, the focusing is kept essentially constant along the cooling channel, though at a beta function somewhat higher than the minimum value achieved in the Study II design. A straightforward Focus-Focus (FOFO) lattice is employed. The solenoidal fields in each half-cell alternate in sign, giving rise to a sinusoidal field variation along the channel.

Use of solid absorbers instead of the liquid-hydrogen absorbers adopted in Study II considerably simplifies the cooling channel, and the new magnet requirements are also more modest, since fewer and weaker components are needed. Together, the Study IIa features reduce the cost of the cooling channel with respect to the Study II design, although, since there is less cooling, a larger acceptance accelerator is required to maintain throughput.

\subsubsection{Acceleration}

Parameters of a representative acceleration system [23] are listed in Table 2. In this design, a matching section using normal-conducting rf systems transforms the cooling channel optics to the optics of the pre-accelerator - a superconducting rf linac with solenoidal focusing that raises the beam energy to 
$1.5 \mathrm{GeV}$. To accommodate the rapid change in energy, the linac is implemented in three parts (see Section 5.2). Figure 5 shows the three cryomodule types that make up the pre-accelerator linac.

This linac is followed by a 3.5-pass "dogbone" RLA (see Fig. 6) that raises the energy from 1.5 to $5 \mathrm{GeV}$. The RLA uses four 2-cell superconducting rf cavity structures per cell, and utilizes quadrupoletriplet rather than solenoidal focusing.

Following the RLA, two cascaded non-scaling FFAG rings increase the beam energy from 5 to $10 \mathrm{GeV}$, and 10 to $20 \mathrm{GeV}$, respectively. Each ring uses combined-function magnets arranged in a triplet (F-D-F) focusing arrangement. The lower energy FFAG ring has a circumference of about $400 \mathrm{~m}$; the higher energy ring is about $500 \mathrm{~m}$ in circumference. Achieving a higher beam energy than $20 \mathrm{GeV}$ would require one or more additional FFAG acceleration stages.

\subsubsection{Storage Ring}

After acceleration in the final FFAG ring, the muons are injected into the upward-going straight section of a racetrack-shaped storage ring with a circumference of $358 \mathrm{~m}$. Parameters of the ring are summarized in Table 3. High-field superconducting arc magnets are used to minimize the arc length and maximize the fraction $(\approx 35 \%)$ of muons that decay in the downward-going straight section, generating neutrinos headed toward the detector located, in the Study IIa design, some $3000 \mathrm{~km}$ away.

The maximum heat load from muon decay electrons is about $45 \mathrm{~kW}(126 \mathrm{~W} / \mathrm{m})$. This load is too high to be dissipated directly in the superconducting coils. There are several ways to deal with this issue. For Study II, a magnet design was chosen that allows the majority of these electrons to exit between separate upper and lower cryostats, and be dissipated in a dump at room temperature. To maintain the vertical cryostat separation in focusing elements, skew quadrupoles are employed in place of standard quadrupoles. Another, equally effective, approach would be to use a liner in the inner bore of a conventional $\cos \theta$ magnet to shield the coils.

Details of the storage ring design are project specific. Different circumferences and different topologies have been studied [30]. Moreover, the storage ring design is not tightly coupled with those of the upstream systems, so considerable flexibility is possible. Note that the footprint of a NF is reasonably small, and such a machine would fit easily on the site of an existing laboratory.

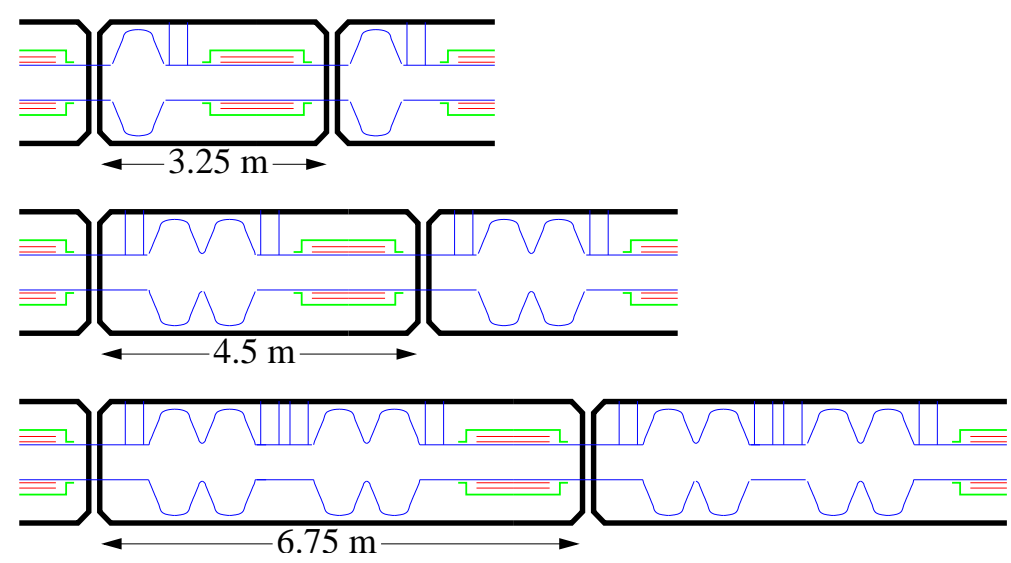

Figure 5: (Color) Layouts of superconducting linac pre-accelerator cryomodules from Ref. [23]. Blue lines are the SC walls of the cavities and solenoid coils are shown in red. The lengths of the cryomodules are indicated. 


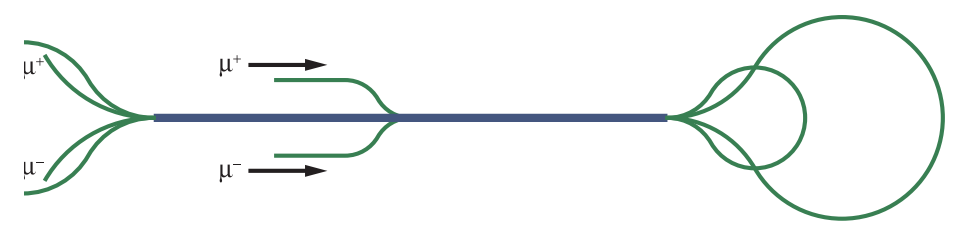

Figure 6: (Color) Layout of the dogbone RLA proposed in [23].

Table 2: Main parameters of the muon accelerator driver from Ref. [23].

\begin{tabular}{lc}
\hline Injection momentum $(\mathrm{MeV} / \mathrm{c})$ & 273 \\
Injection kinetic energy $(\mathrm{MeV})$ & 187 \\
Final total energy $(\mathrm{GeV})$ & 20 \\
Initial normalized acceptance (mm-rad) & 30 \\
$\quad$ Normalized emittance, rms $(\mathrm{mm}-\mathrm{rad})$ & 3.84 \\
Initial longitudinal acceptance, $\Delta p L_{b} / m_{\mu} c(\mathrm{~mm})$ & 150 \\
Total energy spread, $\Delta E(\mathrm{MeV})$ & \pm 45.8 \\
Total pulse length (ns) & \pm 1.16 \\
Energy spread, rms (MeV) & 19.8 \\
Pulse length, rms (ns) & 0.501 \\
Number of bunches per pulse & 89 \\
Peak number of particles per bunch & $1.1 \times 10^{11}$ \\
Number of particles per pulse (per charge) & $3 \times 10^{12}$ \\
Bunch frequency/accelerating frequency $(\mathrm{MHz})$ & $201.25 / 201.25$ \\
Average beam power (per charge) $(\mathrm{kW})$ & 144 \\
\hline
\end{tabular}

\subsection{Ring Configuration Front End}

The scaling-FFAG-based NF design has been studied primarily in Japan [31. The proton driver taken for the design is the J-PARC $50 \mathrm{GeV}$ synchrotron [37]. Compared with the proton driver design described in Section 2.1.1, the beam energy is higher and the repetition rate lower, but the overall beam power, $1 \mathrm{MW}$, is identical. The target and initial capture scheme are very similar to that described in Section 2.1.2. The main difference is that it is envisioned to use a solid target, namely a Ta rod of $1 \mathrm{~cm}$ diameter and $20 \mathrm{~cm}$ length.

Although the scheme described here is, in principle, extendable to higher energies, the present configuration is limited at J-PARC to $20 \mathrm{GeV}$ by site constraints. The NF must fit within the footprint of the $50 \mathrm{GeV}$ J-PARC proton ring and an additional FFAG ring required to reach $50 \mathrm{GeV}$ muon energy would be too large to fulfill this constraint.

Table 3: Representative muon storage ring parameters.

\begin{tabular}{lc}
\hline Energy $(\mathrm{GeV})$ & 20 \\
Circumference $(\mathrm{m})$ & 358.18 \\
Normalized transverse acceptance (mm-rad) & 30 \\
Energy acceptance $(\%)$ & 2.2 \\
Production straight & \\
$\quad$ Length $(\mathrm{m})$ & 126 \\
$\quad \beta_{\max }(\mathrm{m})$ & 200 \\
\hline
\end{tabular}




\subsubsection{Capture and Acceleration}

In the FFAG scenario, the large longitudinal and transverse acceptance of the ring enable the injection of unconditioned beam (no bunching, phase rotation, or cooling). By using a low-frequency (5 MHz) rf system, the rf bucket is capable of accommodating the large momentum spread of the beam, which might be $\Delta p / p \approx 0.5$. The ability to directly capture a large momentum slice obviates the need for developing a momentum correlation along the bunch, so the drift space for this purpose is eliminated in the FFAG design.

Muons are captured into the first-stage FFAG at a central momentum of $0.3 \mathrm{GeV} / \mathrm{c}$ and accelerated to $1 \mathrm{GeV} / \mathrm{c}$. Alternative designs for this ring have been developed based on either normal conducting or superconducting magnets. Ring parameters for both options are summarized in Table 4, taken from Ref. [31]. To avoid the limitations of using H-magnets, which provide little space for injection and extraction, a yoke-free triplet magnet, arranged in a D-F-D configuration, is proposed. A prototype, shown in Fig. 7, has been fabricated and measured.

The second FFAG stage accelerates from 1 to $3 \mathrm{GeV} / \mathrm{c}$. Here too, the ring could be either normalconducting or superconducting, and designs for both options have been developed [31]. The superconducting option produces the smaller ring circumference by nearly a factor of three. The third and fourth stages, operating from 3 to $10 \mathrm{GeV} / \mathrm{c}$ and from 10 to $20 \mathrm{GeV} / \mathrm{c}$, respectively, are both assumed to be superconducting. The reason for this choice is again the J-PARC-specific site constraint of needing to fit within the $50 \mathrm{GeV}$ proton ring footprint. Normal-conducting versions of the stage 3 and 4 FFAG rings are simply too large to fit.

\section{Beam Properties}

The most important neutrino oscillation physics questions that we wish to address in the coming decades require the study of $\nu_{e} \leftrightarrow \nu_{\mu}$ and $\bar{\nu}_{e} \leftrightarrow \bar{\nu}_{\mu}$ transitions in long baseline experiments. Conventional neutrino (antineutrino) beams are almost pure $\nu_{\mu}\left(\bar{\nu}_{\mu}\right)$ beams, which therefore permit the study of $\nu_{\mu} \rightarrow$ $\nu_{e}\left(\bar{\nu}_{\mu} \rightarrow \bar{\nu}_{e}\right)$ oscillations. The experiments using a conventional neutrino beam must therefore look for $\nu_{e}$ Charged Current (CC) interactions in a distant detector. Backgrounds that fake $\nu_{e} \mathrm{CC}$ interactions, together with a small $\nu_{e}$ component in the initial beam, account for $\mathcal{O}(1 \%)$ of the total interaction rate.

Table 4: Main parameters of the 0.3 to $1 \mathrm{GeV} / \mathrm{c}$ FFAG from 31.

\begin{tabular}{lcc}
\hline & normal conducting & superconducting \\
\hline \hline Number of sectors & 32 & 16 \\
$k$ value & 50 & 15 \\
Transition $\gamma$ & 7.1 & 4 \\
Orbit excursion (m) & 0.50 & 0.77 \\
Average radius (m) & 21 & 10 \\
B $@$ F D (T) & 1.8 & 2.8 \\
F/2 opening angle (rad) & 0.026 & 0.052 \\
D opening angle (rad) & 0.018 & 0.036 \\
F/2 bend angle (deg.) & 17 & 26 \\
Packing factor & 0.45 & 0.46 \\
Phase advance, H/V (deg.) & $120 / 61$ & $131 / 103$ \\
Drift length (m) & 2.060 & 2.120 \\
BF length (m) & 1.104 & 1.065 \\
BD length $(m)$ & 0.382 & 0.367 \\
\hline
\end{tabular}




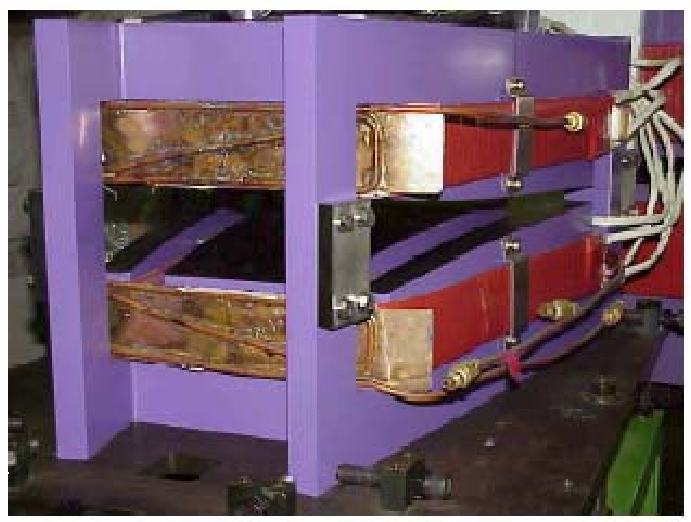

Figure 7: Prototype yoke-free FFAG magnet.

This makes it difficult to probe very small oscillation amplitudes, below the $0.01-0.001$ range. This limitation motivates new types of neutrino facilities that provide $\nu_{e}$ and $\bar{\nu}_{e}$ beams, permitting the search for $\nu_{e} \rightarrow \nu_{\mu}$ and $\bar{\nu}_{e} \rightarrow \bar{\nu}_{\mu}$ oscillations, and if the beam energy is above the $\nu_{\tau}$ CC interaction threshold, the search for $\nu_{e} \rightarrow \nu_{\tau}$ and $\bar{\nu}_{e} \rightarrow \bar{\nu}_{\tau}$ oscillations.

Neutrino Factory beams are produced from muons decaying in a storage ring with long straight sections. Consider an ensemble of polarized negatively-charged muons. When the muons decay they produce muon neutrinos with a distribution of energies and angles in the muon rest frame described by [38]:

$$
\frac{\mathrm{d}^{2} N_{\nu_{\mu}}}{\mathrm{d} x \mathrm{~d} \Omega_{\text {c.m. }}} \propto \frac{2 x^{2}}{4 \pi}\left[(3-2 x)+(1-2 x) P_{\mu} \cos \theta_{\text {c.m. }}\right]
$$

where $x \equiv 2 E_{\nu} / m_{\mu}, \theta_{c . m}$. is the angle between the neutrino momentum vector and the muon spin direction, and $P_{\mu}$ is the average muon polarization along the beam direction. The electron antineutrino distribution is given by:

$$
\frac{\mathrm{d}^{2} N_{\bar{\nu}_{e}}}{\mathrm{~d} x \mathrm{~d} \Omega_{\mathrm{c.m} .}} \propto \frac{12 x^{2}}{4 \pi}\left[(1-x)+(1-x) P_{\mu} \cos \theta_{\text {c.m. }}\right]
$$

and the corresponding distributions for $\bar{\nu}_{\mu}$ and $\nu_{e}$ from $\mu^{+}$decay are obtained by the replacement $P_{\mu} \rightarrow-P_{\mu}$. Only neutrinos and antineutrinos emitted in the forward direction $\left(\cos \theta_{\text {lab }} \simeq 1\right)$ are relevant to the neutrino flux for long-baseline experiments; in this limit, at high energy the maximum $E_{\nu}$ in the laboratory frame is given by:

$$
E_{\max }=\gamma\left[1+\beta \cos \theta_{\text {c.m. }}\right] \frac{m_{\mu}}{2},
$$

where $\beta$ and $\gamma$ are the usual relativistic factors. The $\nu_{\mu}$ and $\bar{\nu}_{e}$ distributions as a function of the laboratory frame variables are then given by:

$$
\frac{\mathrm{d}^{2} N_{\nu_{\mu}}}{\mathrm{d} x \mathrm{~d} \Omega_{\mathrm{lab}}} \propto \frac{1}{\gamma^{2}\left(1-\beta \cos \theta_{\text {lab }}\right)^{2}} \frac{2 x^{2}}{4 \pi}\left[(3-2 x)+(1-2 x) P_{\mu} \cos \theta_{\text {c.m. }}\right],
$$

and

$$
\frac{\mathrm{d}^{2} N_{\bar{\nu}_{e}}}{\mathrm{~d} x \mathrm{~d} \Omega_{\mathrm{lab}}} \propto \frac{1}{\gamma^{2}\left(1-\beta \cos \theta_{\mathrm{lab}}\right)^{2}} \frac{12 x^{2}}{4 \pi}\left[(1-x)+(1-x) P_{\mu} \cos \theta_{\text {c.m. }}\right] .
$$

Thus, for a high energy muon beam with no beam divergence, the neutrino and antineutrino energy and angular distributions depend upon the parent muon energy, the decay angle, and the direction of 
the muon spin vector. With the muon beam intensities that could be provided by a muon collider type muon source [1] the resulting neutrino fluxes at a distant site would be large. For example, Fig. 8 shows, as a function of muon energy and polarization, the computed fluxes per $2 \times 10^{20}$ muon decays at a site on the other side of the Earth $(L=10000 \mathrm{~km})$. Note that the $\nu_{e}\left(\bar{\nu}_{e}\right)$ fluxes are suppressed when the muons have $P=+1(-1)$. This can be understood by examining Eq. (6) and noting that for $P=-1$ the two terms cancel in the forward direction for all $x$. It should be noted that although muons from pion decays are born fully polarized in the pion rest frame, and with substantial polarization in the laboratory frame, they lose polarization due to spin-flip interactions if they pass through matter. Hence NF designs that incorporate an ionization cooling channel are not expected to produce muon beams with high polarization. Nevertheless, Blondel [39] and Fernow et al. [40] have shown that effective polarizations of $\sim 0.3$ might be anticipated.

At low energies, the neutrino $\mathrm{CC}$ interaction cross section is dominated by quasi-elastic scattering and resonance production. However, if $E_{\nu}$ is greater than $\sim 10 \mathrm{GeV}$, the total cross section is dominated by deep inelastic scattering and is approximately [41]:

$$
\begin{aligned}
\sigma\left(\nu+N \rightarrow \ell^{-}+X\right) & \approx 0.67 \times 10^{-38} \times E_{\nu}(\mathrm{GeV}) \mathrm{cm}^{2} \\
\sigma\left(\bar{\nu}+N \rightarrow \ell^{+}+X\right) & \approx 0.34 \times 10^{-38} \times E_{\bar{\nu}}(\mathrm{GeV}) \mathrm{cm}^{2}
\end{aligned}
$$

The number of $\nu$ and $\bar{\nu} \mathrm{CC}$ events per incident neutrino observed in an isoscalar target is given by:

$$
\begin{aligned}
& N\left(\nu+N \rightarrow \ell^{-}+X\right)=4.0 \times 10^{-15} \times E_{\nu}(\mathrm{GeV}) \text { events per } \mathrm{g} / \mathrm{cm}^{2}, \\
& N\left(\bar{\nu}+N \rightarrow \ell^{+}+X\right)=2.0 \times 10^{-15} \times E_{\bar{\nu}}(\mathrm{GeV}) \text { events per } \mathrm{g} / \mathrm{cm}^{2}
\end{aligned}
$$

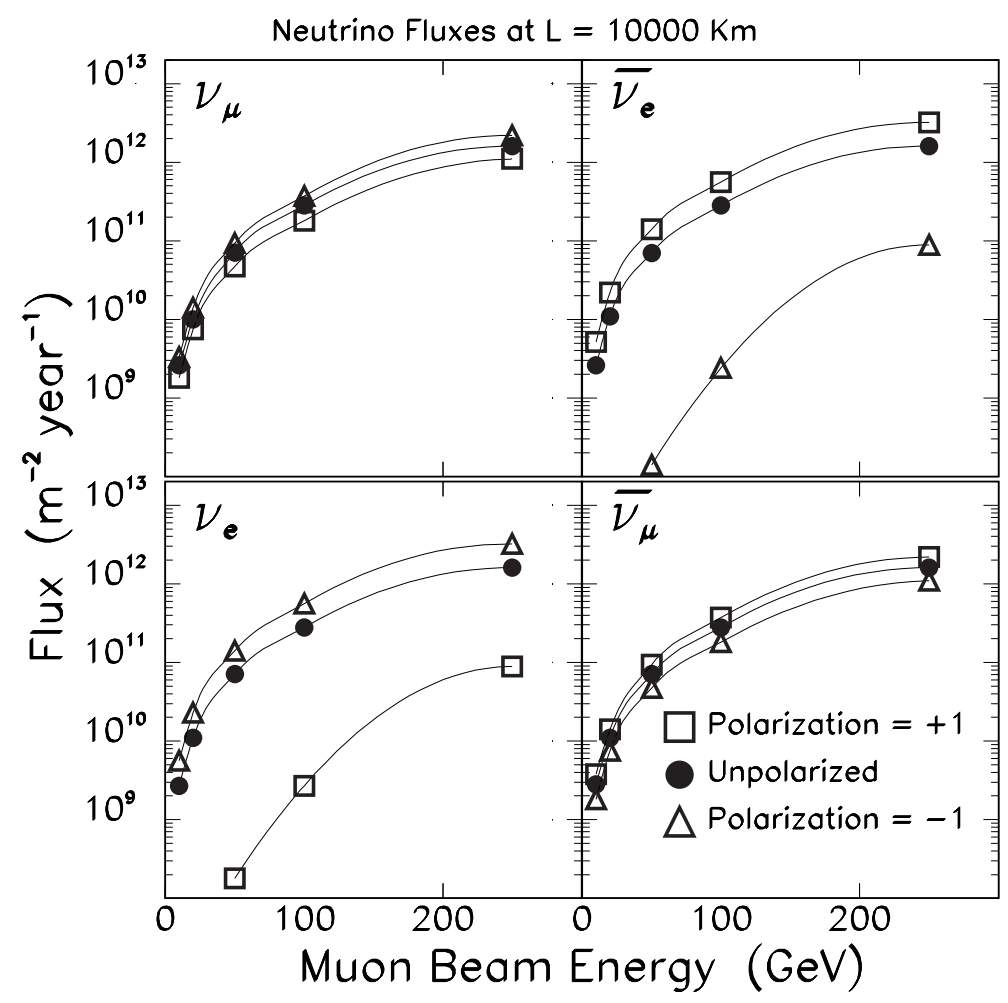

Figure 8: Calculated $\nu$ and $\bar{\nu}$ fluxes in the absence of oscillations at a far site located $10000 \mathrm{~km}$ from a NF in which $2 \times 10^{20}$ muons have decayed in the storage ring straight section pointing at the detector. The fluxes are shown as a function of the energy of the stored muons for negative muons (top two plots) and positive muons (bottom two plots), and for three muon polarizations as indicated. The calculated fluxes are averaged over a circular area of radius $1 \mathrm{~km}$ at the far site. Calculation from Ref. [3]. 
Using this simple form for the energy dependence of the cross section, the predicted energy distributions for $\nu_{e}$ and $\nu_{\mu}$ interacting in a far detector $(\cos \theta=1)$ at a NF are shown in Fig. 9. The interacting $\nu_{\mu}$ energy distribution is compared in Fig. 10 with the corresponding distribution arising from the highenergy NuMI [42] wide-band beam. Note that neutrino beams from a NF have no high energy tail, and in that sense can be considered narrow-band beams.

In practice, $\mathrm{CC}$ interactions can only be cleanly identified when the final state lepton exceeds a threshold energy. The calculated final state lepton distributions are shown in Fig. 11. Integrating over the energy distribution, the total $\nu$ and $\bar{\nu}$ interaction rates per muon decay are given by:

$$
N_{\nu}=1.2 \times 10^{-14}\left[\frac{E_{\mu}^{3}(\mathrm{GeV})}{L^{2}(\mathrm{~km})}\right] \times C(\nu) \text { events per kton }
$$

and

$$
N_{\bar{\nu}}=0.6 \times 10^{-14}\left[\frac{E_{\mu}^{3}(\mathrm{GeV})}{L^{2}(\mathrm{~km})}\right] \times C(\nu) \text { events per kton }
$$

where

$$
C\left(\nu_{\mu}\right)=\frac{7}{10}+P_{\mu} \frac{3}{10} \quad, \quad C\left(\nu_{e}\right)=\frac{6}{10}-P_{\mu} \frac{6}{10} .
$$

The calculated $\nu_{e}$ and $\nu_{\mu} \mathrm{CC}$ interaction rates resulting from $2 \times 10^{20}$ muon decays in the straight section of a NF are compared in Table 5, for several different energies and baselines, with expectations for the corresponding rates for some representative experiments using conventional neutrino beams. Note that event rates at a NF increase as $E_{\mu}^{3}$. The event rates at a $10 \mathrm{GeV} \mathrm{NF}$ are comparable to the corresponding conventional beam rates, and become significantly larger than the conventional neutrino

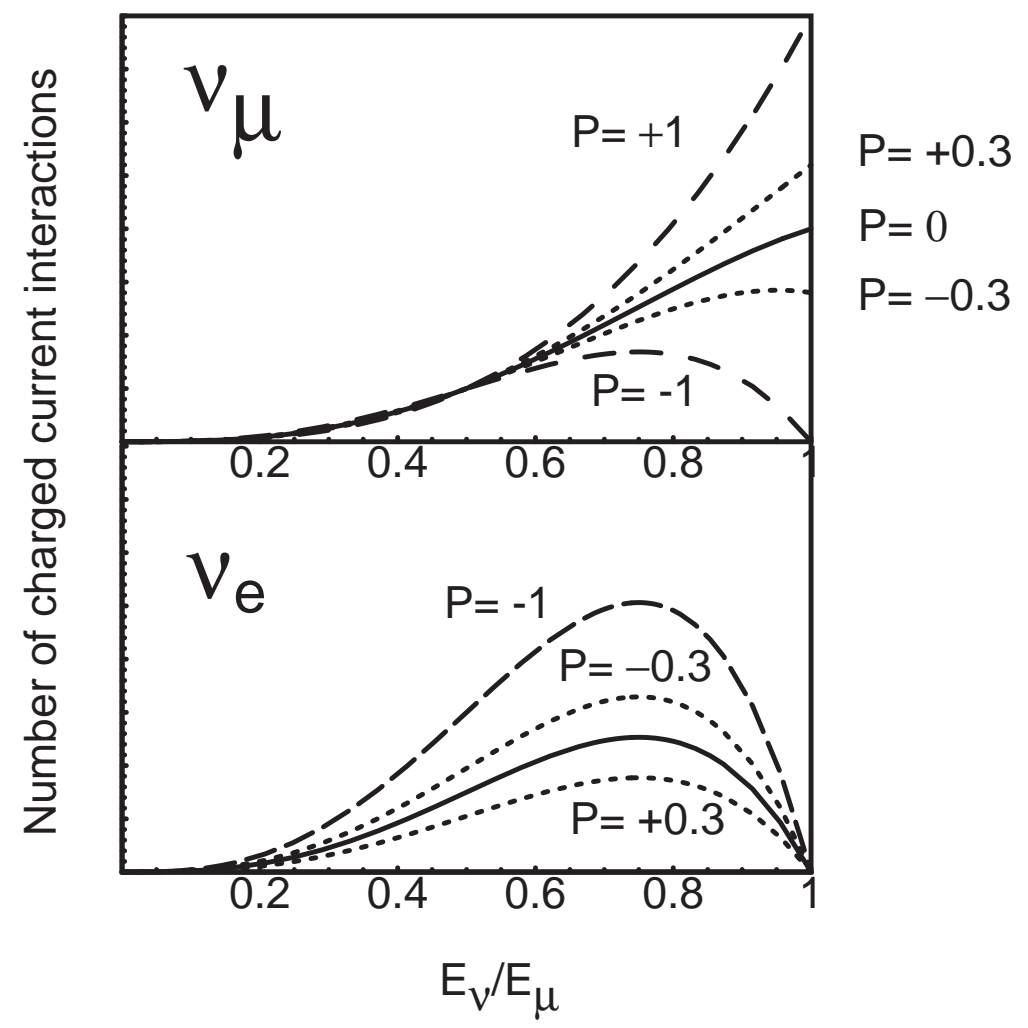

Figure 9: Charged current event spectra at a far detector. The solid lines indicate zero polarization, the dotted lines indicate polarization of \pm 0.3 and the dashed lines indicate full polarization. The $P=1$ case for electron neutrinos results in no events and is hidden by the $x$ axis. Figure from Ref. [20]. 

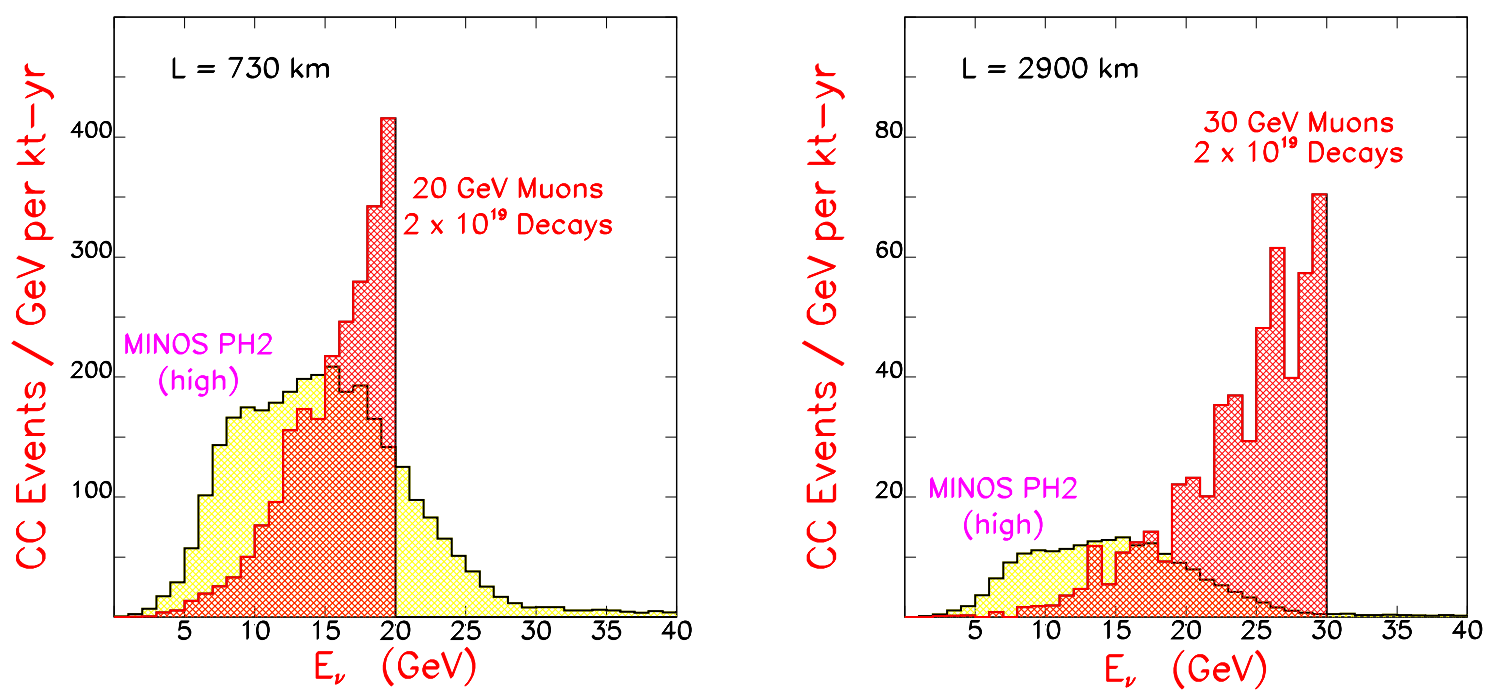

Figure 10: Comparison of interacting $\nu_{\mu}$ energy distributions for the NuMI high energy wide-band beam (Ref. [42]) with those for a $20 \mathrm{GeV} \mathrm{NF}$ beam (Ref. [3]) at $L=730 \mathrm{~km}$, and a $30 \mathrm{GeV} \mathrm{NF}$ beam at $L=2900 \mathrm{~km}$. The NF distributions have been calculated based on Eq. (2) (no approximations), and include realistic muon beam divergences and energy spreads. The NF rates are for $2 \times 10^{19}$ muon decays, which corresponds to about 1 month of running. Figure from Ref. [20].

beam rates for $\mathrm{NF}$ energies in excess of $\sim 10 \mathrm{GeV}$. The radial dependence of the event rate is shown in Fig. 12 for a $20 \mathrm{GeV} \mathrm{NF}$ and three baselines.

The systematic uncertainties on the neutrino flux at a NF have been discussed in Refs. [44; 45]. Since muon decay kinematics is very well understood, and the beam properties of the muons in the storage ring can be well determined, we expect the systematic uncertainties on the neutrino beam intensity and spectrum to be small compared with the corresponding uncertainties on the properties of conventional neutrino beams. In the muon decay straight section of a NF, the muon beam is designed to have an average divergence given by $\sigma_{\theta}=\mathcal{O}\left(\frac{0.1}{\gamma}\right)$. The neutrino beam divergence will therefore be dominated by muon decay kinematics, and uncertainties on the beam direction and divergence will yield only small uncertainties in the neutrino flux at a far site. However, if precise knowledge of the flux is required, the uncertainties on $\theta$ and $\sigma_{\theta}$ must be taken into account, along with uncertainties on the flux arising from uncertainties on the muon energy distribution and polarization. The relationships between the uncertainties on the muon beam properties and the resulting uncertainties on the neutrino flux are summarized in Table 6. If, for example, we wish to know the $\nu_{e}$ and $\nu_{\mu}$ fluxes at a far site with a precision of $1 \%$, we must determine the beam divergence, $\sigma_{\theta}$, to $20 \%$, and ensure that the beam direction is within $0.6 \times \sigma_{\theta}$ of the nominal direction [44]. We point out that it should be possible to do much better than this, and consequently, to know the fluxes at the far site with a precision much better than $1 \%$.

We now consider the event distributions in a detector at a near site, close to the NF, which will be quite different from the corresponding distributions at a far site. There are two main reasons for this difference. First, the near detector accepts neutrinos over a large range of muon decay angles $\theta$, not just those neutrinos traveling in the extreme forward direction. This results in broader neutrino and antineutrino energy distributions that are sensitive to the radial size of the detector (Fig. 13). Second, if the distance of the near detector from the end of the decay straight section is of the order of the straight section length, then the $\theta$ acceptance of the detector varies with the position of the muon decay along the straight section. This results in a more complicated radial flux distribution than expected at a far detector. However, since the dominant effects arise from the decay length and muon decay kinematics, 


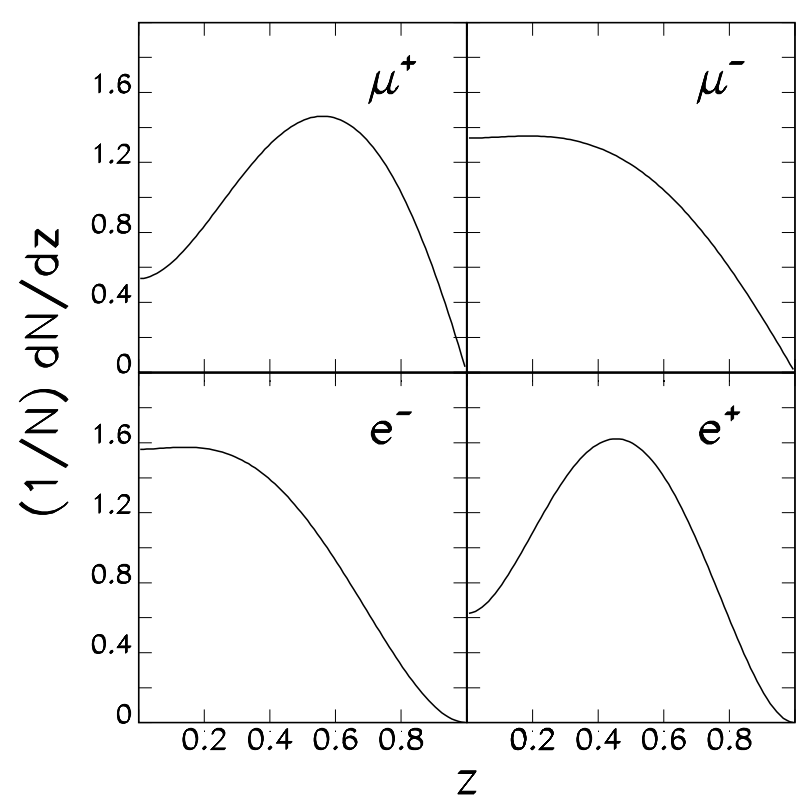

Figure 11: Lepton energy spectra for $\mathrm{CC} \bar{\nu}_{\mu}$ (top left), $\nu_{\mu}$ (top right), $\nu_{e}$ (bottom left), and $\bar{\nu}_{e}$ (bottom right) interactions. Note that $z$ is the energy normalized to the primary muon energy $z=E_{\ell} / E_{\mu}$. Calculation from Ref. [43].

the flux distribution should be modeled quite accurately (Fig. 14). Note that, even in a limited angular range, the event rates in a near detector are very high. Figure 15 illustrates the event rates per $\mathrm{g} / \mathrm{cm}^{2}$ as a function of energy. Because most of the neutrinos produced forward in the center of mass traverse the near detector fiducial volume, the effective flux does not substantially increase for higher energy neutrino factories, and the event rate therefore increases approximately linearly with $E_{\mu}$. For a $50 \mathrm{GeV}$ muon storage ring, the interaction rate per $2 \times 10^{20}$ muon decays is $1.4 \times 10^{7}$ events per g/ $\mathrm{cm}^{2}$. Finally, in the absence of special magnetized shielding, the high neutrino event rates in any material upstream of the detector will cause substantial backgrounds. The event rate in the last three interaction lengths $\left(300 \mathrm{~g} / \mathrm{cm}^{2}\right)$ of the shielding between the detector and the storage ring would be 30 interactions per beam spill at a $15 \mathrm{~Hz}$ machine delivering $2 \times 10^{20}$ muon decays per year. These high background rates will require clever magnetized shielding designs and fast detector readout to avoid overly high accidental rates.

\section{Physics Reach}

In the following a brief review is given of the theoretical and phenomenological framework used to describe neutrino oscillation physics, together with a summary of our present knowledge of the neutrino oscillation parameters. The physics sensitivity that would be provided by a NF is then discussed.

\subsection{Theoretical Framework}

There are three known flavors of active neutrinos which, within the Standard Model (SM), form lefthanded doublets with their associated charged leptons. In principle, there can be additional flavors of neutrino which are singlets under the electroweak gauge group. If they exist, these electroweak singlet neutrinos do not have electroweak couplings, and their interactions are not described by the SM. If $\nu=\left(\nu_{e}, \nu_{\mu}, \nu_{\tau}\right)$ is the flavor vector of the $\mathrm{SU}(2) \times \mathrm{U}(1)$ active neutrinos, and $\chi=\left(\chi_{1}, . ., \chi_{n_{s}}\right)$ is the 
Table 5: Muon neutrino and electron antineutrino CC interaction rates in the absence of oscillations, calculated for baseline length $L=732 \mathrm{~km}$ (FNAL $\rightarrow$ Soudan), for MINOS using the wide-band beam and a muon storage ring delivering $2 \times 10^{20}$ decays with $E_{\mu}=10,20$, and $50 \mathrm{GeV}$ at three baselines. The NF calculation includes a realistic muon beam divergence and energy spread. Table from Ref. [20].

\begin{tabular}{|c|c|c|c|c|c|c|}
\hline Experiment & & $\begin{array}{c}\text { Baseline } \\
(\mathrm{km})\end{array}$ & $\begin{array}{l}\left\langle E_{\nu_{\mu}}\right\rangle \\
(\mathrm{GeV})\end{array}$ & $\begin{array}{c}\left\langle E_{\bar{\nu}_{e}}\right\rangle \\
(\mathrm{GeV})\end{array}$ & $\begin{array}{c}\mathrm{N}\left(\nu_{\mu} \mathrm{CC}\right) \\
\text { (per kton-yr) }\end{array}$ & $\begin{array}{c}\mathrm{N}\left(\bar{\nu}_{e} \mathrm{CC}\right) \\
\text { (per kton-yr) }\end{array}$ \\
\hline \multirow[t]{3}{*}{ MINOS } & Low energy & 732 & 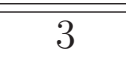 & - & 458 & 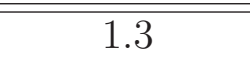 \\
\hline & Medium energy & 732 & 6 & - & 1439 & 0.9 \\
\hline & High energy & 732 & 12 & - & 3207 & 0.9 \\
\hline \multirow[t]{4}{*}{ Muon ring } & $E_{\mu}(\mathrm{GeV})$ & & & & & \\
\hline & 10 & 732 & 7.5 & 6.6 & 2800 & 1240 \\
\hline & 20 & 732 & 15 & 13 & 24000 & 10000 \\
\hline & 50 & 732 & 38 & 33 & $3.6 \times 10^{5}$ & $1.5 \times 10^{5}$ \\
\hline \multirow[t]{4}{*}{ Muon ring } & $E_{\mu}(\mathrm{GeV})$ & & & & & \\
\hline & 10 & 2900 & 7.6 & 6.5 & 182 & 82 \\
\hline & 20 & 2900 & 15 & 13 & 1480 & 660 \\
\hline & 50 & 2900 & 38 & 33 & 22000 & 9800 \\
\hline \multirow[t]{4}{*}{ Muon ring } & $E_{\mu}(\mathrm{GeV})$ & & & & & \\
\hline & 10 & 7300 & 7.5 & 6.4 & 28 & 12 \\
\hline & 20 & 7300 & 15 & 13 & 220 & 102 \\
\hline & 50 & 7300 & 38 & 33 & 3800 & 1540 \\
\hline
\end{tabular}

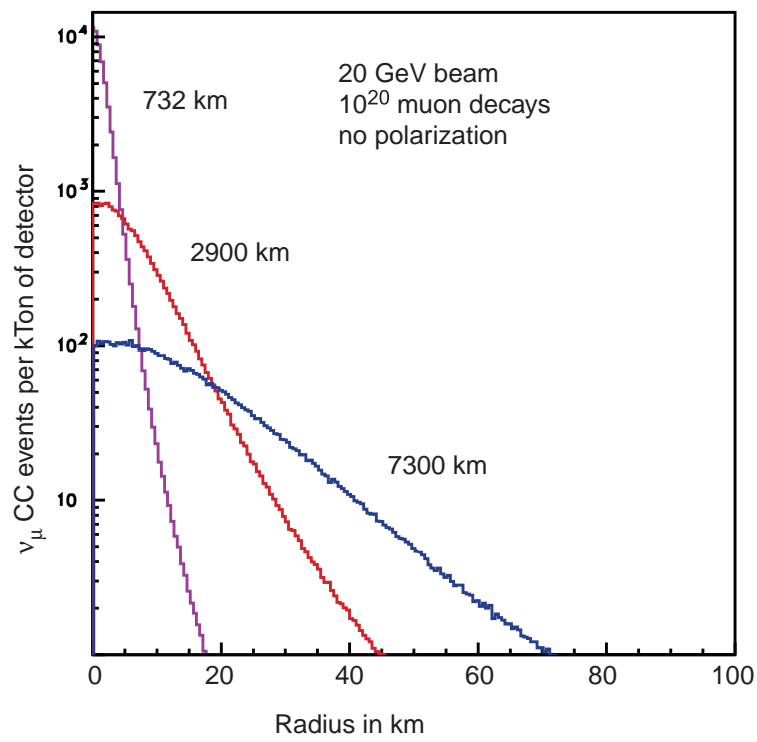

Figure 12: Events per kton of detector as a function of distance from the beam axis for a $20 \mathrm{GeV}$ muon beam. Figure from Ref. [20]. The rates are for $10^{20}$ muon decays, which corresponds to 6 months of running. 
Table 6: Dependence of predicted charged current event rates on muon beam properties at a NF. The last column lists the required precisions with which each beam property must be determined if the uncertainty on the neutrino flux at the far site is to be less than $\sim 1 \%$. Here $\Delta$ denotes uncertainty while $\sigma$ denotes the spread in a variable. Table from Ref. [44].

\begin{tabular}{cccc}
\hline $\begin{array}{c}\text { Muon beam } \\
\text { property }\end{array}$ & $\begin{array}{c}\text { Beam } \\
\text { type }\end{array}$ & $\begin{array}{c}\text { Rate } \\
\text { dependence }\end{array}$ & $\begin{array}{c}\text { Target } \\
\text { precision }\end{array}$ \\
\hline \hline Energy, $E_{\mu}$ & $\nu$ (no osc.) & $\begin{array}{c}\frac{\Delta N}{N}=3 \frac{\Delta E_{\mu}}{E_{\mu}} \\
\frac{\Delta E_{\mu}}{E_{\mu}}<0.003 \\
\frac{\Delta N}{N}=2 \frac{\Delta E_{\mu}}{E_{\mu}}\end{array}$ & $\frac{\Delta E_{\mu}}{E_{\mu}}<0.005$ \\
\hline Direction, $\Delta \theta$ & $\nu$ (no osc.) & $\frac{\Delta N}{N} \leq 0.01$ & $\Delta \theta<0.6 \sigma_{\theta}$ \\
& & for $\left.\Delta \theta<0.6 \sigma_{\theta}\right)$ & \\
\hline Divergence, $\sigma_{\theta}$ & $\nu$ (no osc.) & $\frac{\Delta N}{N} \sim 0.03 \frac{\Delta \sigma_{\theta}}{\sigma_{\theta}}$ & $\frac{\Delta \sigma_{\theta}}{\sigma_{\theta}}<0.2$ \\
$\left(\right.$ for $\left.\sigma_{\theta} \sim 0.1 / \gamma\right)$ & $\left(\right.$ for $\left.\sigma_{\theta} \sim 0.1 / \gamma\right)$ \\
\hline Momentum spread, $\sigma_{p}$ & $\nu$ (no osc.) & $\frac{\Delta N}{N} \sim 0.06 \frac{\Delta \sigma_{p}}{\sigma_{p}}$ & $\frac{\Delta \sigma_{p}}{\sigma_{p}}<0.17$ \\
\hline Polarization, $P_{\mu}$ & $\nu_{e}$ (no osc.) & $\frac{\Delta N_{\nu_{e}}}{N_{\nu_{e}}}=\Delta P_{\mu}$ & $\Delta P_{\mu}<0.01$ \\
& $\nu_{\mu}$ (no osc.) & $\frac{\Delta N_{\nu_{\mu}}}{N_{\nu_{\mu}}}=0.4 \Delta P_{\mu}$ & $\Delta P_{\mu}<0.025$ \\
\hline
\end{tabular}

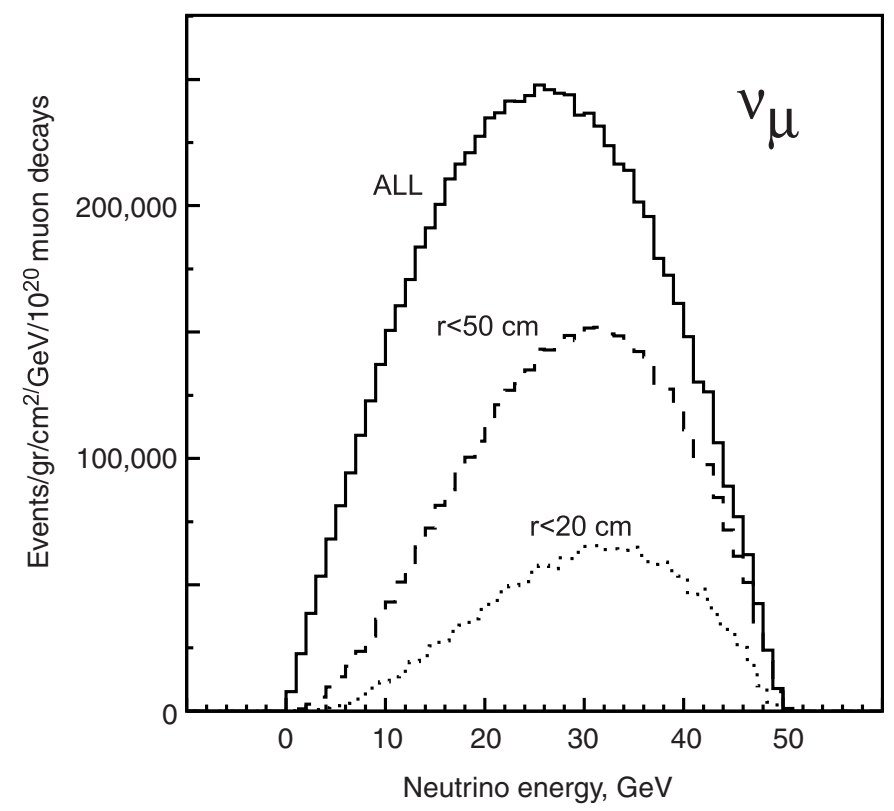

Figure 13: Events per $\mathrm{g} / \mathrm{cm}^{2}$ per $\mathrm{GeV}$ for a detector $40 \mathrm{~m}$ from a muon storage ring with a $600 \mathrm{~m}$ straight section. The three curves show all events and those falling within 50 and $20 \mathrm{~cm}$ of the beam axis. The rates are for $10^{20}$ muon decays, which corresponds to 6 months running. Figure from Ref. [20]. 


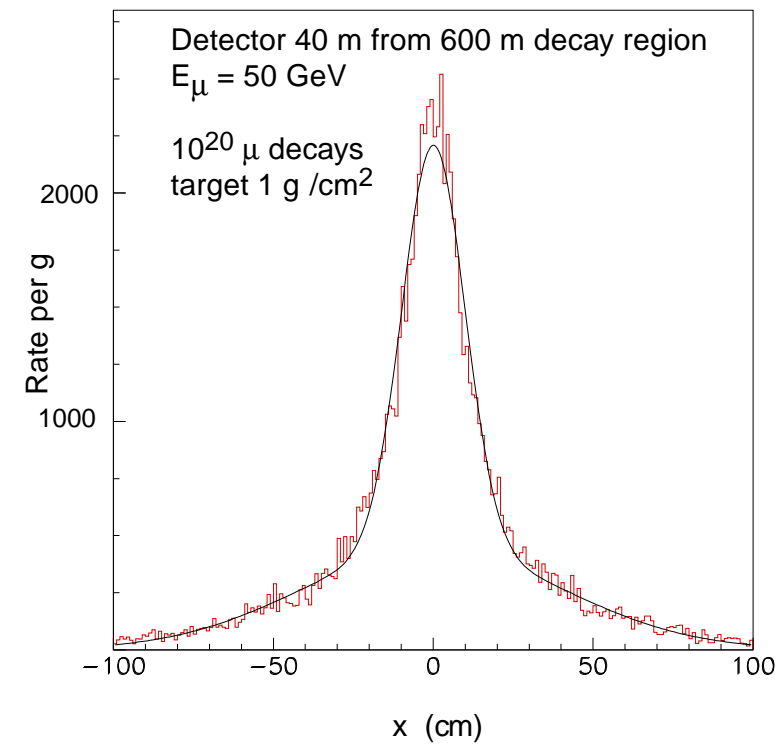

Figure 14: Events per $\mathrm{g}$ as a function of the transverse coordinate, $x, 40 \mathrm{~m}$ downstream of a $50 \mathrm{GeV}$ NF providing $10^{20}$ muon decays (corresponding to 6 months running). The central peak is mainly due to decays in the last $100 \mathrm{~m}$ of the decay pipe, while the large tails are due to upstream decays. Figure from Ref. [20].

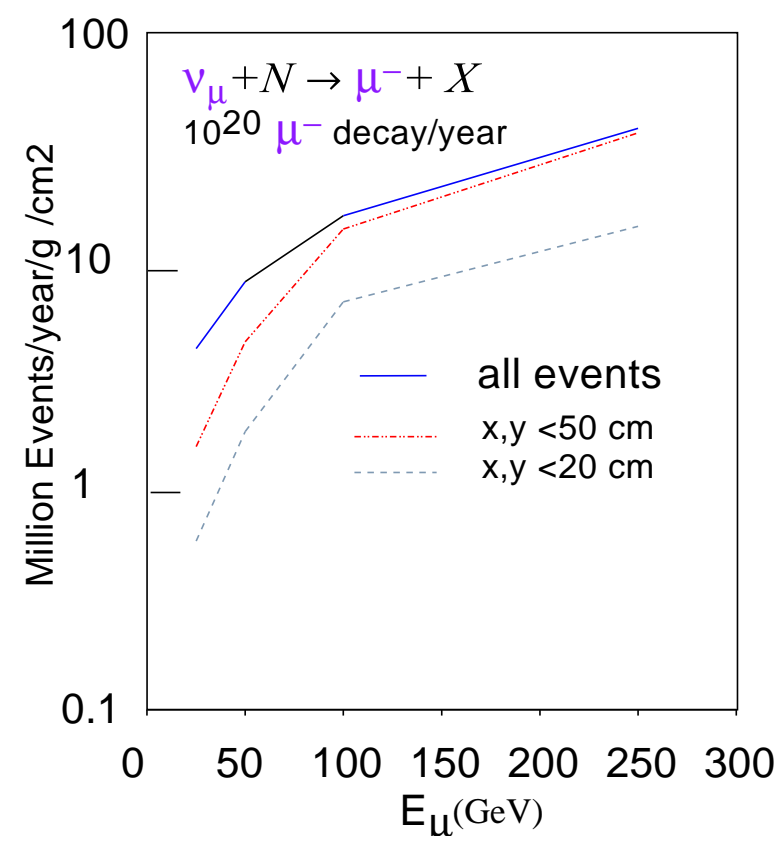

Figure 15: Events per year and per $\mathrm{g} / \mathrm{cm}^{2}$ at a near detector as a function of muon beam energy in $\mathrm{GeV}$. The solid curves indicate all events, the dashed and dotted curves show the effects of radial position cuts. This assumes $10^{20}$ muon decays/year. The numbers should be doubled for $2 \times 10^{20}$ decays per year. Figure from Ref. [20]. 
vector of electroweak-singlet neutrinos, the Dirac and Majorana neutrino mass terms can be written as:

$$
-\mathcal{L}_{m}=\frac{1}{2}\left(\bar{\nu}_{L} \bar{\chi}_{L}^{c}\right)\left(\begin{array}{cc}
M_{L} & M_{D} \\
\left(M_{D}\right)^{T} & M_{R}
\end{array}\right)\left(\begin{array}{c}
\nu_{R}^{c} \\
\chi_{R}
\end{array}\right)+\text { h.c. }
$$

where $M_{L}$ is the $3 \times 3$ left-handed Majorana mass matrix, $M_{R}$ is an $n_{s} \times n_{s}$ right-handed Majorana mass matrix, and $M_{D}$ is the 3-row by $n_{s}$-column Dirac mass matrix. In general, all of these are complex, and $\left(M_{L}\right)^{T}=M_{L}, \quad\left(M_{R}\right)^{T}=M_{R}$. Without further theoretical input, the number $n_{s}$ of "sterile" electroweak-singlet neutrinos is not determined. For example, in the SM, minimal supersymmetric standard model (MSSM), or minimal SU(5) grand unified theory (GUT), $n_{s}=0$, while in the $\mathrm{SO}(10)$ GUT, $n_{s}=3$.

The most natural explanation for the three known ultra-light neutrino masses is generally regarded to be the seesaw mechanism [46; 47; 48; 49; 50; $51 ; 52$; 53], which involves $M_{R}$, and arises from Eq. (14) in the case of $n_{s}=3$ electroweak singlet neutrinos. This leads to neutrino masses that are of order

$$
m_{\nu} \sim \frac{m_{D}^{2}}{m_{R}}
$$

where $m_{D}$ and $m_{R}$ denote typical elements of the corresponding matrices. With $m_{D} \sim m_{t}$ and $m_{R} \sim$ $10^{16} \mathrm{GeV}$, as suggested in a (supersymmetric) SO(10) GUT framework, a scale of $m_{\nu} \sim 10^{-3} \mathrm{eV}$ is readily obtained. In this case, the three light neutrino masses are obtained by diagonalization of the effective $3 \times 3$ light neutrino mass matrix

$$
M_{\nu}=-M_{D} M_{R}^{-1} M_{D}^{T}
$$

while the super-heavy neutrinos are determined from the right-handed Majorana matrix $M_{R}$.

\subsection{Phenomenological Framework}

Since transitions have been observed between the three flavor eigenstates, we now know that neutrinos have non-zero masses, and that there is mixing between the flavor eigenstates. The mass eigenstates $\nu_{i}=\left(\nu_{1}, \nu_{2}, \nu_{3}\right)$ with masses $m_{i}=\left(m_{1}, m_{2}, m_{3}\right)$ are related to the flavor eigenstates by a $3 \times 3$ unitary mixing matrix $U^{\nu}[54]$,

$$
\left|\nu_{\alpha}\right\rangle=\sum_{i}\left(U_{\alpha i}^{\nu}\right)^{*}\left|\nu_{i}\right\rangle
$$

where $\nu_{\alpha}=\left(\nu_{e}, \nu_{\mu}, \nu_{\tau}\right)$. Four numbers are needed to specify all of the matrix elements, namely three mixing angles $\left(\theta_{12}, \theta_{23}, \theta_{13}\right)$ and one complex phase $(\delta)$. In terms of these parameters:

$$
U^{\nu}=\left(\begin{array}{ccc}
c_{13} c_{12} & c_{13} s_{12} & s_{13} e^{-i \delta} \\
-c_{23} s_{12}-s_{13} s_{23} c_{12} e^{i \delta} & c_{23} c_{12}-s_{13} s_{23} s_{12} e^{i \delta} & c_{13} s_{23} \\
s_{23} s_{12}-s_{13} c_{23} c_{12} e^{i \delta} & -s_{23} c_{12}-s_{13} c_{23} s_{12} e^{i \delta} & c_{13} c_{23}
\end{array}\right)
$$

where $c_{j k} \equiv \cos \theta_{j k}$ and $s_{j k} \equiv \sin \theta_{j k}$. Neutrino oscillation measurements have already provided some knowledge of $U^{\nu}$, which is approximately given by:

$$
U^{\nu}=\left(\begin{array}{ccc}
0.8 & 0.5 & ? \\
0.4 & 0.6 & 0.7 \\
0.4 & 0.6 & 0.7
\end{array}\right)
$$

We have limited knowledge of the $(1,3)$-element of the mixing matrix, which is parametrized by $s_{13} e^{-i \delta}$. We have only an upper limit on $\theta_{13}$ and no knowledge of $\delta$. Note that $\theta_{13}$ and $\delta$ are particularly important because if $\theta_{13}$ and $\sin \delta$ are non-zero there is $C P$ violation in the neutrino sector. 
Neutrino oscillations are driven by the splittings between the neutrino mass eigenstates. It is useful to define the differences between the squares of the masses of the mass eigenstates $\Delta m_{i j}^{2} \equiv m_{i}^{2}-m_{j}^{2}$. The probability that a neutrino of energy $E$ and initial flavor $\alpha$ will "oscillate" into a neutrino of flavor $\beta$ is given by $P_{\alpha \beta} \equiv P\left(\nu_{\alpha} \rightarrow \nu_{\beta}\right)=\left|\left\langle\nu_{\beta}|\exp (-i \mathcal{H} t)| \nu_{\alpha}\right\rangle\right|^{2}$, which in vacuum is given by

$$
P_{\alpha \beta}=\sum_{j=1}^{3} \sum_{k=1}^{3} U_{\alpha j} U_{\alpha k}^{*} U_{\beta j}^{*} U_{\beta k} \exp \left(-i \frac{\Delta m_{k j}^{2}}{2 E} t\right) .
$$

If neutrinos of energy $E$ travel a distance $L$, then a non-zero $\Delta m_{i j}^{2}$ will result in neutrino flavor oscillations that have maxima at given values of $L / E$, and oscillation amplitudes that are determined by the matrix elements $U_{\alpha i}^{\nu}$, and hence by $\theta_{12}, \theta_{23}, \theta_{13}$, and $\delta$. Equation 20 can be rewritten:

$$
P\left(\nu_{\alpha} \rightarrow \nu_{\beta}\right)=P_{C P \text {-even }}\left(\nu_{\alpha} \rightarrow \nu_{\beta}\right)+P_{C P \text {-odd }}\left(\nu_{\alpha} \rightarrow \nu_{\beta}\right)
$$

where the $C P$-even and $C P$-odd contributions are

$$
\begin{aligned}
P_{C P \text {-even }}\left(\nu_{\alpha} \rightarrow \nu_{\beta}\right) & =P_{C P \text {-even }}\left(\bar{\nu}_{\alpha} \rightarrow \bar{\nu}_{\beta}\right) \\
& =\delta_{\alpha \beta}-4 \sum_{i>j} \Re\left(U_{\alpha i} U_{\beta i}^{*} U_{\alpha j}^{*} U_{\beta j}\right) \sin ^{2}\left(\frac{\Delta m_{i j}^{2} L}{4 E}\right) \\
P_{C P \text {-odd }}\left(\nu_{\alpha} \rightarrow \nu_{\beta}\right) & =-P_{C P \text {-odd }}\left(\bar{\nu}_{\alpha} \rightarrow \bar{\nu}_{\beta}\right) \\
& =2 \sum_{i>j} \Im\left(U_{\alpha i} U_{\beta i}^{*} U_{\alpha j}^{*} U_{\beta j}\right) \sin \left(\frac{\Delta m_{i j}^{2} L}{2 E}\right)
\end{aligned}
$$

so that

$$
P\left(\bar{\nu}_{\alpha} \rightarrow \bar{\nu}_{\beta}\right)=P\left(\nu_{\beta} \rightarrow \nu_{\alpha}\right)=P_{C P \text {-even }}\left(\nu_{\alpha} \rightarrow \nu_{\beta}\right)-P_{C P \text {-odd }}\left(\nu_{\alpha} \rightarrow \nu_{\beta}\right)
$$

where, by $C P T$ invariance, $P\left(\nu_{\alpha} \rightarrow \nu_{\beta}\right)=P\left(\bar{\nu}_{\beta} \rightarrow \bar{\nu}_{\alpha}\right)$. In vacuum, the $C P$-even and $C P$-odd contributions are even and odd, respectively, under time reversal: $\alpha \leftrightarrow \beta$. In Eq. (22), the combination $\Delta m_{i j}^{2} L /(4 E)$ in $\hbar=c=1$ units can be replaced by $1.267 \Delta m_{i j}^{2} L / E$ with $\Delta m_{i j}^{2}$ in $\mathrm{eV}^{2}$ and $(L, E)$ in $(\mathrm{km}, \mathrm{GeV})$. In disappearance experiments, $\beta=\alpha$ and no $C P$-violation can appear since the product of the mixing matrix elements is inherently real.

If the neutrinos propagate through matter, the expressions for the oscillation probabilities must be modified. The propagation of neutrinos through matter is described by the evolution equation

$$
i \frac{\mathrm{d} \nu_{\alpha}}{\mathrm{d} t}=\sum_{\beta}\left[\left(\sum_{j} U_{\alpha j} U_{\beta j}^{*} \frac{m_{j}^{2}}{2 E_{\nu}}\right)+\frac{A}{2 E_{\nu}} \delta_{\alpha e} \delta_{\beta e}\right] \nu_{\beta},
$$

where $A /\left(2 E_{\nu}\right)$ is the amplitude for coherent forward CC scattering of $\nu_{e}$ on electrons,

$$
A=2 \sqrt{2} G_{F} N_{e} E_{\nu}=1.52 \times 10^{-4} \mathrm{eV}^{2} Y_{e} \rho\left(\mathrm{g} / \mathrm{cm}^{3}\right) E(\mathrm{GeV})
$$

(for $\overline{\nu_{e}} A$ is replaced with $-A$ ). Here $Y_{e}$ is the electron fraction and $\rho$ is the matter density. Density profiles through the earth can be calculated using the Earth Model [55], and are shown in Fig. 16. For neutrino trajectories through the earth's crust, the density is typically of order $3 \mathrm{~g} / \mathrm{cm}^{3}$, and $Y_{e} \simeq 0.5$. For very long baselines a constant density approximation is not sufficient and oscillation calculations must explicitly take account of the density profile. However, the constant density approximation is very useful to understand the physics of neutrinos propagating through the earth since, in practice, the variation of the density is not large.

The propagation Eq. (24) can be re-expressed in terms of mass-squared differences:

$$
i \frac{\mathrm{d} \nu_{\alpha}}{\mathrm{d} t}=\sum_{\beta} \frac{1}{2 E_{\nu}}\left[\Delta m_{31}^{2} U_{\alpha 3} U_{\beta 3}^{*}+\Delta m_{21}^{2} U_{\alpha 2} U_{\beta 2}^{*}+A \delta_{\alpha e} \delta_{\beta e}\right] \nu_{\beta} .
$$




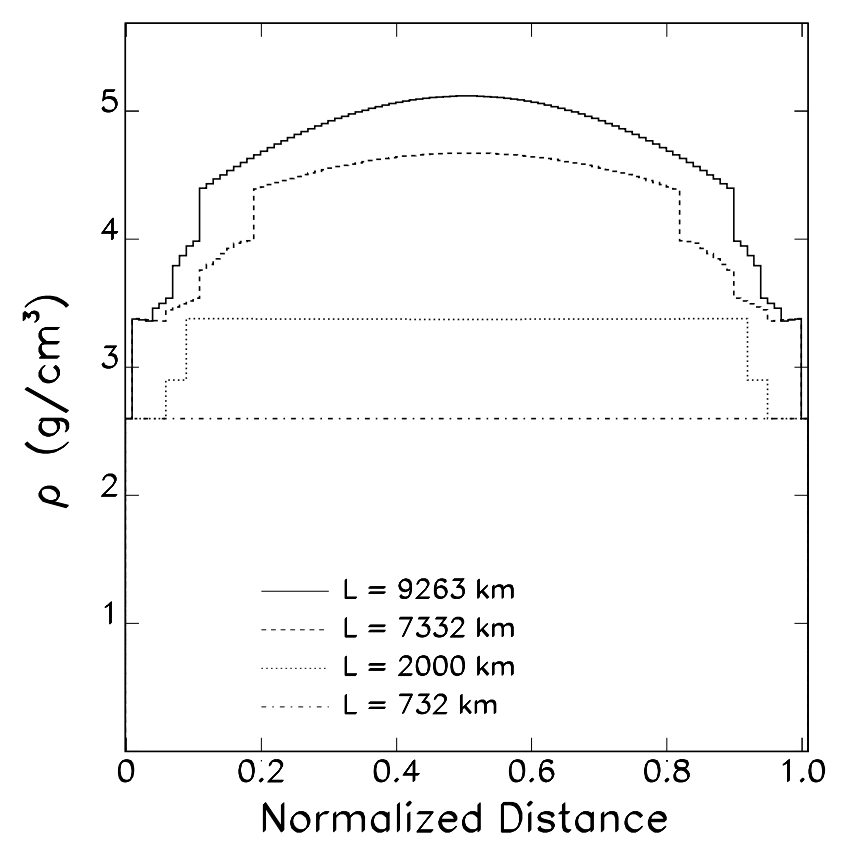

Figure 16: Density profiles for trajectories through the Earth. Calculation from Ref. [43].

This evolution equation can be solved numerically for given input values of the $\Delta m_{i j}^{2}$ and mixing matrix elements.

For long-baseline experiments, the appearance probability $\nu_{e} \rightarrow \nu_{\mu}$ in matter can be expanded in the small hierarchy parameter $\alpha \equiv \Delta m_{21}^{2} / \Delta m_{31}^{2}$ and the small $\sin 2 \theta_{13}$ regime up to the second order as [56; $57 ;$ 58]:

$$
\begin{aligned}
P_{e \mu} & \simeq \sin ^{2} 2 \theta_{13} \sin ^{2} \theta_{23} \frac{\sin ^{2}[(1-\hat{A}) \Delta]}{(1-\hat{A})^{2}} \\
& \pm \alpha \sin 2 \theta_{13} \xi \sin \delta_{\mathrm{CP}} \sin (\Delta) \frac{\sin (\hat{A} \Delta)}{\hat{A}} \frac{\sin [(1-\hat{A}) \Delta]}{(1-\hat{A})} \\
& +\alpha \sin 2 \theta_{13} \xi \cos \delta_{\mathrm{CP}} \cos (\Delta) \frac{\sin (\hat{A} \Delta)}{\hat{A}} \frac{\sin [(1-\hat{A}) \Delta]}{(1-\hat{A})} \\
& +\alpha^{2} \cos ^{2} \theta_{23} \sin ^{2} 2 \theta_{12} \frac{\sin ^{2}(\hat{A} \Delta)}{\hat{A}^{2}} .
\end{aligned}
$$

Here $\Delta \equiv \Delta m_{31}^{2} L /(4 E), \xi \equiv \cos \theta_{13} \sin 2 \theta_{12} \sin 2 \theta_{23}$, and $\hat{A} \equiv \pm\left(2 \sqrt{2} G_{F} n_{e} E\right) / \Delta m_{31}^{2}$ with $G_{F}$ the Fermi coupling constant and $n_{e}$ the electron density in matter. The sign of the second term is determined by choosing $\nu_{e} \rightarrow \nu_{\mu}$ (positive) or $\bar{\nu}_{e} \rightarrow \bar{\nu}_{\mu}$ (negative) as the oscillation channel, and the sign of $\hat{A}$ is determined by the sign of $\Delta m_{31}^{2}$ and choosing neutrinos or antineutrinos. This formula shows that close to the resonance condition, $\hat{A} \simeq 1$, the first term can be enhanced by matter effects. Therefore, it is most affected by the sign of $\Delta m_{31}^{2}$. In addition, $C P$ effects are only present in the second and third terms. Depending on which quantity should be measured, one or two of the terms will act as signal and the rest of the terms as background. The formula also indicates that the values of $\sin 2 \theta_{13}$ and the hierarchy parameter $\alpha$ change the relative weight of the individual terms, which means that in certain regions of the $\sin ^{2} 2 \theta_{13}$ - $\alpha$-plane the measurements corresponding to the selected terms will be favored. For example, the $C P$ violation measurements are easier if both $\alpha$ and $\theta_{13}$ are large, whereas if $\alpha$ is small the sign of $\Delta m_{31}^{2}$ is easier to determine. 

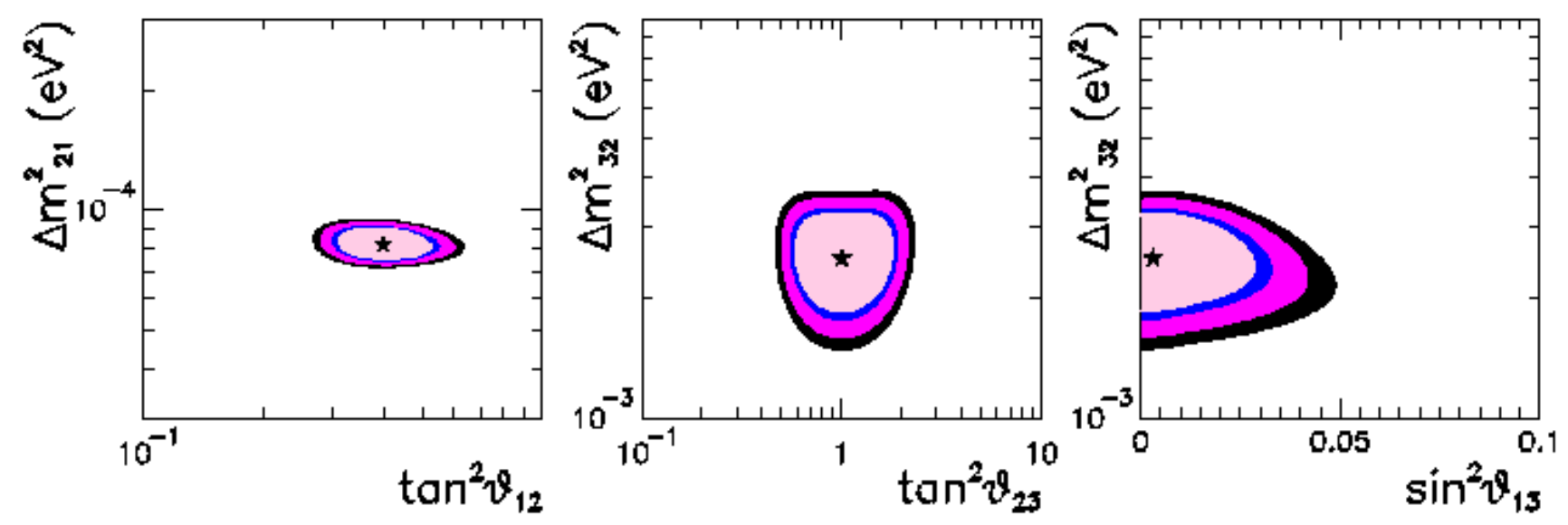

Figure 17: Current experimental constraints on the three mixing angles $\theta_{12}, \theta_{23}$, and $\theta_{13}$ and on the two mass-squared differences $\Delta m_{21}^{2}$ and $\Delta m_{32}^{2}$. The star indicates the most likely solution. Figure taken from [59].

\subsection{Present Experimental Status}

Our present knowledge of the neutrino mass splittings and mixing matrix has been obtained from atmospheric [17; 61], solar [62; 63; 64; 65; 66; 67], reactor [68; 69; 70], and accelerator-based [71] neutrino experiments, and is summarized in Fig. 17. The solar-neutrino experiments and the reactor experiment KamLAND probe values of $L / E$ that are sensitive to $\Delta m_{21}^{2}$, and the mixing angle $\theta_{12}$. Our knowledge of these parameters is shown in the left panel of Fig. 17. The atmospheric-neutrino experiments and the accelerator based experiment $\mathrm{K} 2 \mathrm{~K}$ probe values of $L / E$ that are sensitive to $\Delta m_{32}^{2}$, and the mixing angle $\theta_{23}$. Our knowledge of these parameters is shown in the central panel of Fig. 17. Searches for $\nu_{\mu} \leftrightarrow \nu_{e}$ transitions with values of $L / E$ corresponding to the atmospheric-neutrino scale are sensitive to the third mixing angle $\theta_{13}$. To date these searches have not observed this transition, and hence we have only an upper limit on $\theta_{13}$, which comes predominantly from the CHOOZ reactor experiment [68], and is shown in the right panel of Fig. 17.

The mixing angles tell us about the flavor content of the neutrino mass eigenstates. Our knowledge of the $\Delta m_{i j}^{2}$ and the flavor content of the mass eigenstates is summarized in Fig. 18. Note that there are two possible patterns of neutrino mass. This is because the neutrino oscillation experiments to date have been sensitive to the magnitude of $\Delta m_{32}^{2}$, but not its sign. The neutrino spectrum shown on the left in Fig. 18 is called the Normal Mass Hierarchy and corresponds to $\Delta m_{32}^{2}>0$. The neutrino spectrum shown on the right is called the Inverted Mass Hierarchy and corresponds to $\Delta m_{32}^{2}<0$.

Finally, it should be noted that there is a possible complication to the simple three-flavor neutrino oscillation picture. The LSND [72] experiment has reported evidence for muon anti-neutrino to electron anti-neutrino transitions for values of $L / E$ that are two orders of magnitude smaller than the corresponding values observed for atmospheric neutrinos. The associated transition probability is very small, of the order of $0.3 \%$. If this result is confirmed by the MiniBooNE [73] experiment, it will require a third characteristic $L / E$ range for neutrino flavor transitions. Since each $L / E$ range implies a different mass-splitting between the participating neutrino mass eigenstates, confirmation of the LSND result would require more than three mass eigenstates. This would be an exciting and radical development. Independent of whether the LSND result is confirmed, it is important that the future global neutrino oscillation program makes further tests of the three-flavor oscillation framework. 


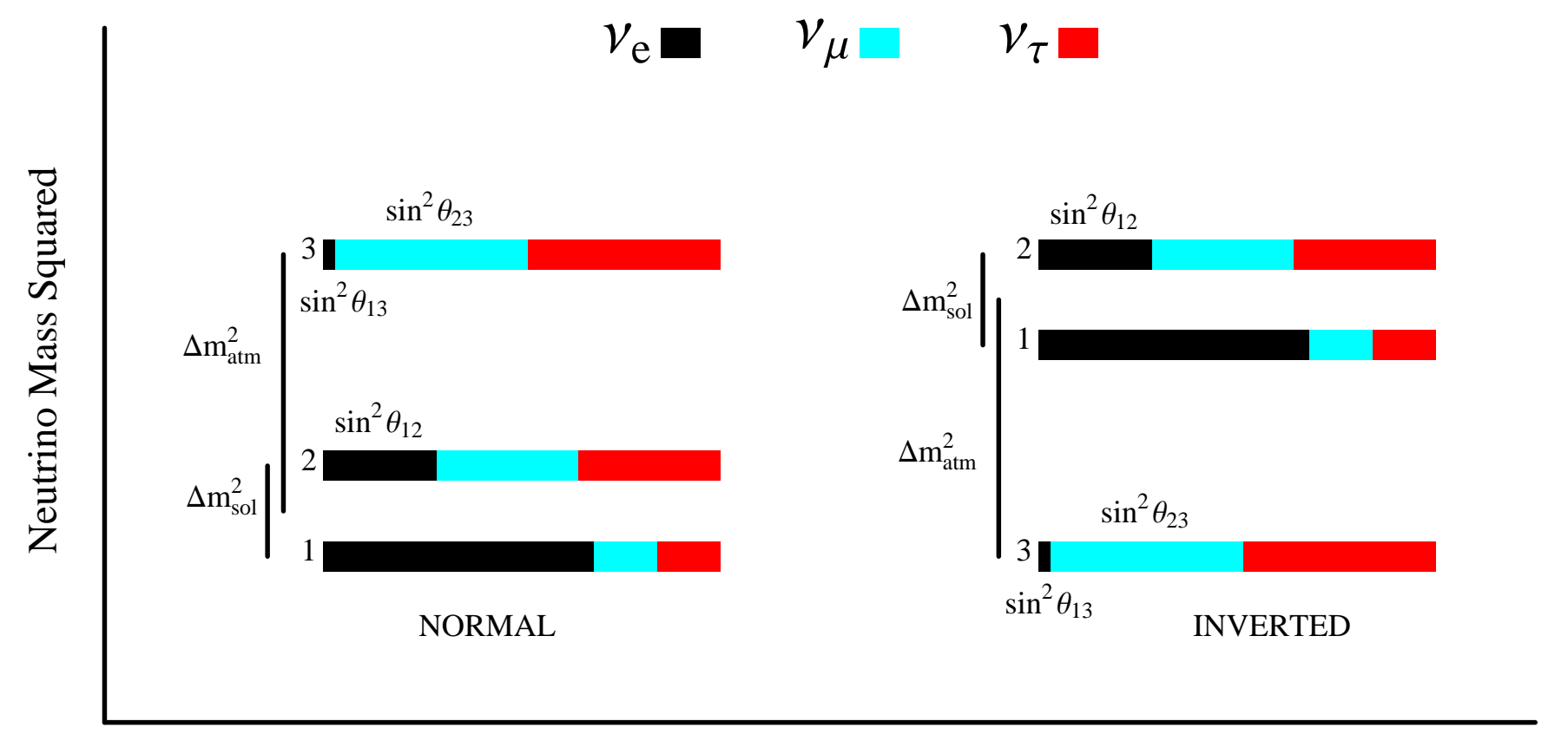

Fractional Flavor Content

Figure 18: The two possible arrangements of the masses of the three known neutrinos, based on neutrino oscillation measurements. The spectrum on the left corresponds to the Normal Hierarchy and has $\Delta m_{32}^{2}>0$. The spectrum on the right corresponds to the Inverted Hierarchy and has $\Delta m_{32}^{2}<0$. The $\nu_{e}$ fraction of each mass eigenstate is indicated by the black solid region. The $\nu_{\mu}$ and $\nu_{\tau}$ fractions are indicated by the blue (red) regions, respectively. The $\nu_{e}$ fraction in the mass eigenstate labeled " 3 " has been set to the CHOOZ bound. Figure from Ref. [60].

\subsection{Neutrino Factory Sensitivity}

Given our present knowledge of neutrino masses and mixings, the most important questions to be addressed by future accelerator-based neutrino experiments have been identified as [59]:

(i) What is the value of the unknown mixing angle $\theta_{13}$ ? Is it extremely small, which might suggest a new flavor symmetry in the lepton sector?

(ii) Is the mass hierarchy normal or inverted? Specific neutrino mass models are usually only compatible with one of the two possible neutrino mass hierarchies. A measurement of the sign of $\Delta m_{31}^{2}$ would discriminate between models. For example, GUT models with a standard type I see-saw mechanism tend to predict a normal hierarchy (see, for example, the reviews in Refs. [74; 75]), while an inverted hierarchy is often obtained in models that employ flavor symmetries such as $L_{e}-L_{\mu}-L_{\tau}[76 ; 77$.

(iii) Is there observable $C P$ violation in the neutrino sector and what is the value of the Dirac phase $\delta$ ? These measurements may provide clues about the origin of the observed matter-antimatter asymmetry in the universe.

To address these questions will require measuring $\nu_{e} \leftrightarrow \nu_{\mu}$ transitions. At a NF $\nu_{e} \rightarrow \nu_{\mu}$ transitions result in "wrong-sign" muons, a particularly clean experimental signature that would facilitate very sensitive measurements. 


\subsubsection{Wrong-Sign Muons, Detector Considerations, and Event Rates}

At a NF in which, for example, positive muons are stored, the initial beam consists of $50 \% \nu_{e}$ and $50 \%$ $\bar{\nu}_{\mu}$. In the absence of oscillations, the $\nu_{e} \mathrm{CC}$ interactions produce electrons and the $\bar{\nu}_{\mu} \mathrm{CC}$ interactions produce positive muons. Note that the charge of the final state lepton tags the flavor of the initial $\nu$ or $\bar{\nu}$. In the presence of $\nu_{e} \rightarrow \nu_{\mu}$ oscillations, the $\nu_{\mu} \mathrm{CC}$ interactions produce negative muons (i.e., wrong-sign muons). This is a very clean experimental signature since it is straightforward to suppress backgrounds to 1 part in $10^{4}$ of the total CC interaction rate, or better. Thus, at a NF, backgrounds to the $\nu_{e} \rightarrow \nu_{\mu}$ oscillation signal are extremely small. The full statistical sensitivity can therefore be exploited down to values of $\sin ^{2} 2 \theta_{13}$ approaching $10^{-4}$ before backgrounds must be subtracted and further advances in sensitivity scale like $\sqrt{N}$ rather than $N$. This enables Neutrino Factories to go beyond the sensitivities achievable by conventional neutrino beams by about two orders of magnitude.

In a neutrino experiment, the statistical sensitivity depends upon the product of the beam flux, running time, and detector fiducial mass. In the literature, different authors have taken as reasonable a range of values for these three factors. In many studies, beam fluxes corresponding to $2 \times 10^{20}$ muon decays per year have been assumed. More recently, improved NF designs suggest this flux might be higher by a factor of a few. Typically, running periods between 5 years and 10 years have been considered. The assumed detector mass has typically been between $\mathcal{O}(10$ kton $)$ and $\mathcal{O}(100$ kton $)$, and depends critically upon the detector technology being considered.

Since a NF detector must be able to identify and measure muons, and distinguish positive from negative muons, it must be magnetized. Many studies have considered, as a representative NF detector, a magnetized (MINOS-like) steel/scintillator calorimeter. The detector would consist of iron plates interspersed with scintillator planes. Transverse position information can be obtained by segmenting the scintillator within each plane. With reasonable segmentation, the transverse resolution is dominated by scattering and the detector performance depends mainly on its longitudinal segmentation, which must be fine enough to determine whether a particle has penetrated beyond the region of any accompanying hadronic shower. If it has, the penetrating track is a muon candidate. The momentum resolution is determined by the magnetic field and the thickness of the steel plates. The energy of fully contained muons can also be measured from their range. Note that very low energy muons do not have a sufficiently long path length to discriminate them from leading hadrons within a hadronic shower. Iron/scintillator calorimeters therefore effectively have a muon energy threshold of a few GeV. In studies to date, this threshold has been taken to be at about $4 \mathrm{GeV}$. With very fine segmentation the threshold might be significantly reduced, but this is still under study. One advantage of using a magnetized steel/scintillator calorimeter is that very massive detectors can be considered, and fiducial masses of typically 50 kton have been assumed in the literature.

A liquid-argon calorimeter within a magnetic field, or combined with a magnetic spectrometer to determine muon charge, has also been considered as a NF detector. The effective muon threshold for this attractive option appears to be around $2 \mathrm{GeV}$. Hence, this technology promises higher efficiency for low energy neutrino interactions, which compensates for the assumed smaller fiducial masses that are considered reasonable (typically 20-30 kton).

In both iron/scintillator and liquid-argon NF detectors, backgrounds to wrong-sign muons can be produced in $\mathrm{NC}$ and $\mathrm{CC}$ interactions by $(i)$ pions or kaons from a hadronic shower that decay to produce a muon, (ii) non-interacting pions (punch-through), and (iii) charm-meson production with muonic decay. These backgrounds can be suppressed with simple cuts, including a muon energy threshold cut. The $4 \mathrm{GeV}(2 \mathrm{GeV})$ minimum muon energy "cuts" for iron/scintillator (liquid-argon) NF detectors are expected to suppress backgrounds to the $10^{-4}$ level, or better. At the present time, more work is needed to understand the most cost-effective combination of NF performance, detector technology ${ }^{1}$ and mass,

\footnotetext{
${ }^{1}$ Recent studies have suggested it might be possible to reduce the muon threshold further using a fully active detector within a large magnetic volume. New concepts for producing a cost-effective large magnetic volume are being explored 78 .
} 
and running time. In the meantime, the results based on various combinations that are summarized in the following section, should be considered as representative, and not necessarily optimal.

Very low background rates enable very small oscillation amplitudes, and hence very small values of $\theta_{13}$, to be probed provided the event rates are adequate. Wrong-sign muon event rates at a representative NF experiment are compared in Table 7 with $\nu_{\mu} \rightarrow \nu_{e}$ signal rates for various experiments using a conventional neutrino beam (see Table 8). Experiments using conventional neutrino beams can expect at most a few hundred $\nu_{\mu} \rightarrow \nu_{e}$ events with a background of $\mathcal{O}(10 \%)$. If $\theta_{13}$ is significantly below the present upper limit, the signal will be less and the signal/background correspondingly worse. If $\sin ^{2} 2 \theta_{13}$ is less than $\sim 0.01-0.02$ it will be difficult to observe $\nu_{\mu} \rightarrow \nu_{e}$ oscillations with high statistical confidence using a conventional neutrino beam, and even more difficult to determine the mass hierarchy and search for $C P$ violation. In contrast, at a NF the signal samples would be $\mathcal{O}\left(10^{4}\right)$ events for the largest values of $\theta_{13}$. If $\theta_{13}$ is an order of magnitude below the present upper limit $\left(\sin ^{2} 2 \theta_{13} \sim 0.01\right)$ the wrong-sign muon signal samples would still be $\mathcal{O}\left(10^{3}\right)$ events with backgrounds of a few percent. The backgrounds are sufficiently small and the signal rates sufficiently large to enable a NF to probe values of $\sin ^{2} 2 \theta_{13}$ down to $\mathcal{O}\left(10^{-4}-10^{-5}\right)$.

We now consider how wrong-sign muon measurements at a NF can be used to answer the most important neutrino oscillation physics questions. Suppose we store positive muons in a $50 \mathrm{GeV} \mathrm{NF}$, and measure the number of events tagged by a negative muon in a detector at $L=3000 \mathrm{~km}$, and then store negative muons and measure the rate of events tagged by a positive muon. The calculated signal rates are compared in Table 9 for the normal and inverted mass hierarchies and for $\delta=0$ (no $C P$ violation) and $\delta=\pi / 2$ (maximal $C P$ violation). The signal rates are sensitive to the parameter values. Furthermore the data from the $\mu^{+}$and $\mu^{-}$running complement each other in a way that helps to uniquely determine the parameter values.

The expected NF signal rates depend not only on the oscillation parameters, but also on the NF energy and the experiment baseline. In Fig. 19 the predicted ratio of wrong-sign muon events $R \equiv$ $N\left(\bar{\nu}_{e} \rightarrow \bar{\nu}_{\mu}\right) / N\left(\nu_{e} \rightarrow \nu_{\mu}\right)$ that corresponds to $50 \% \mu^{+}$running and $50 \% \mu^{-}$running is shown for a $20 \mathrm{GeV} \mathrm{NF}$ as a function of baseline for $\Delta m_{32}^{2}=+0.0035 \mathrm{eV}^{2}$ and $-0.0035 \mathrm{eV}^{2}$, with $\sin ^{2} 2 \theta_{13}$ set to the small value 0.004. Although the $\Delta m_{32}^{2}$ values used for Fig. 19 (taken from Ref. [82]) are now a little different from those emerging from global analyses of the atmospheric and solar neutrino data, they nonetheless serve to illustrate how the measurements can be used to determine the oscillation

Table 7: Signal and background $\nu_{\mu} \rightarrow \nu_{e}$ and $\nu_{e} \rightarrow \nu_{\mu}$ event rates at, respectively, conventional neutrino beam experiments and an experiment at a $50 \mathrm{GeV}$ NF. Rates are shown for values of $\theta_{13}$ that are just below the present upper limit and an order of magnitude below the upper limit. The rates are for the normal mass hierarchy and $\delta=0$. The numbers for each experiment correspond to 5 years of running with the nominal beam intensities and "reasonable" detector masses. Parameters for the conventional beam experiments are listed in Table 8. The calculations are from W. Winter, and are based on the Globes program [79]. Table from Ref. [80].

\begin{tabular}{lccc}
\hline Experiment & $\begin{array}{c}\text { Signal } \\
\sin ^{2} 2 \theta_{13}=0.1\end{array}$ & $\begin{array}{c}\text { Signal } \\
\sin ^{2} 2 \theta_{13}=0.01\end{array}$ & Background \\
\hline \hline MINOS & 49.1 & 6.7 & 108 \\
OPERA & 11.2 & 1.6 & 28.3 \\
T2K & 132 & 16.9 & 22.7 \\
NO $\nu \mathrm{A}$ & 186 & 23.0 & 19.7 \\
NO $\nu$ A $+\mathrm{FPD}$ & 716 & 88.6 & 75.6 \\
$\mathrm{NF}\left(\nu_{e} \rightarrow \nu_{\mu}\right)$ & 29752 & 4071 & 44.9 \\
$\mathrm{NF}\left(\bar{\nu}_{e} \rightarrow \bar{\nu}_{\mu}\right)$ & 7737 & 1116 & 82.0 \\
\hline
\end{tabular}


Table 8: Parameters describing conventional neutrino beam and reactor experiments referred to in the text, figures, and tables. The parameters listed are the baseline $L$, the mean neutrino energy $\left\langle E_{\nu}\right\rangle$, the source integrated intensity or power $P_{\text {Source }}$ (for beams: in protons on target, pot, per year, for reactors: in gigawatts of thermal reactor power), the detector technology, the fiducial detector mass $m_{\text {Det }}$, and the running time $t_{\text {run }}$. Table modified from Ref [81].

\begin{tabular}{|c|c|c|c|c|c|c|}
\hline Label & $L$ & $\left\langle E_{\nu}\right\rangle$ & $P_{\text {Source }}$ & Detector technology & $m_{\text {Det }}$ & $t_{\text {run }}$ \\
\hline \multicolumn{7}{|c|}{ Conventional beam experiments: } \\
\hline MINOS & $735 \mathrm{~km}$ & $3 \mathrm{GeV}$ & $3.7 \times 10^{20} \mathrm{pot} / \mathrm{y}$ & Magn. iron calor & 5.4 kton & $5 y$ \\
\hline OPERA & $732 \mathrm{~km}$ & $17 \mathrm{GeV}$ & $4.5 \times 10^{19} \mathrm{pot} / \mathrm{y}$ & Emul. cloud chamb. & 1.65 kton & $5 y$ \\
\hline \multicolumn{7}{|c|}{ Off-Axis experiments: } \\
\hline $\mathrm{T} 2 \mathrm{~K}$ & $295 \mathrm{~km}$ & $0.76 \mathrm{GeV}$ & $1.0 \times 10^{21} \mathrm{pot} / \mathrm{y}$ & Water Cherenkov & 22.5 kton & $5 y$ \\
\hline $\mathrm{NO} \nu \mathrm{A}^{\mathrm{a})}$ & $812 \mathrm{~km}$ & $2.22 \mathrm{GeV}$ & $4.0 \times 10^{20} \mathrm{pot} / \mathrm{y}$ & Low-Z-calorimeter & $30 \mathrm{kt}$ & $5 y$ \\
\hline \multicolumn{7}{|c|}{ Reactor experiments: } \\
\hline D-Chooz & $1.05 \mathrm{~km}$ & $\sim 4 \mathrm{MeV}$ & $2 \times 4.25 \mathrm{GW}$ & Liq & 11. & $3 y$ \\
\hline Reactor-Il ${ }^{\mathrm{b})}$ & $1.70 \mathrm{~km}$ & $\sim 4 \mathrm{MeV}$ & $8 \mathrm{GW}$ & Liquid scintillator & 200 ton & $5 \mathrm{yr}$ \\
\hline
\end{tabular}

a) proposed

Table 9: Signal and background $\nu_{\mu} \rightarrow \nu_{e}$ and $\nu_{e} \rightarrow \nu_{\mu}$ event rates at, respectively, conventional neutrino beam experiments (see Table 8) and an experiment at a $50 \mathrm{GeV} \mathrm{NF}(L=3000 \mathrm{~km})$. Rates are shown for values of $\theta_{13}$ that are just below the present upper limit $\left(\sin ^{2} 2 \theta_{13}=0.1\right)$. The rates are for the normal and inverted mass hierarchies with $\delta=0$ (no $C P$ violation) and $\delta=\pi / 2$ (maximal $C P$ violation). The numbers for each experiment correspond to 5 years of running with the nominal beam intensities and "reasonable" detector masses. The calculations are from W. Winter, and are based on the Globes program [79]. Table from Ref. [80].

\begin{tabular}{lrrrrr}
\hline Experiment & $\begin{array}{r}\text { Normal } \\
\delta=0\end{array}$ & $\begin{array}{r}\text { Normal } \\
\delta=\pi / 2\end{array}$ & $\begin{array}{r}\text { Inverted } \\
\delta=0\end{array}$ & $\begin{array}{r}\text { Inverted } \\
\delta=\pi / 2\end{array}$ & $\begin{array}{r}\text { Back- } \\
\text { ground }\end{array}$ \\
\hline \hline $\mathrm{T} 2 \mathrm{~K}$ & 132 & 96 & 102 & 83 & 22.7 \\
$\mathrm{NO} \nu \mathrm{A}$ & 186 & 138 & 111 & 85 & 19.7 \\
$\mathrm{NO} \nu \mathrm{A}+\mathrm{FPD}$ & 716 & 531 & 430 & 326 & 75.6 \\
$\mathrm{NF}\left(\nu_{e} \rightarrow \nu_{\mu}\right)$ & 29752 & 27449 & 13060 & 17562 & 44.9 \\
$\mathrm{NF}\left(\bar{\nu}_{e} \rightarrow \bar{\nu}_{\mu}\right)$ & 7737 & 5942 & 9336 & 10251 & 82.0 \\
\hline
\end{tabular}

parameters. Figure 19 shows two bands. The upper (lower) band corresponds to $\Delta m_{32}^{2}<0(>0)$. Within the bands, the $C P$ phase $\delta$ is varying. At short baselines the bands converge, and the ratio $R=0.5$ since the antineutrino CC cross section is half of the neutrino CC cross section. At large distances, matter effects enhance $R$ if $\Delta m_{32}^{2}<0$ and reduce $R$ if $\Delta m_{32}^{2}>0$, and the bands diverge. Matter effects become significant for baselines exceeding about $2000 \mathrm{~km}$. The error bars indicate the expected statistical uncertainty on the measured $R$ with a data sample of $5 \times 10^{22}$ kton-decays, which corresponds to a few years of running with a reasonably large detector. With these statistics, the sign of $\Delta m_{32}^{2}$ is determined with very high statistical significance. With an order of magnitude smaller data sample (entry level scenario [83]) or with an order of magnitude smaller $\sin ^{2} 2 \theta_{13}$ the statistical uncertainties would be $\sqrt{10}$ larger, but the sign of $\Delta m_{32}^{2}$ could still be determined with convincing precision. In addition to the ratio of wrong-sign muon signal rates $R$, the two wrong-sign muon event energy distributions can be separately measured. To show how this additional information can help, the predicted measured energy distributions $2800 \mathrm{~km}$ downstream from a $30 \mathrm{GeV}$ NF are shown in Figs. 20 and 21 for, respectively, $\nu_{e} \rightarrow \nu_{\mu}$ and $\bar{\nu}_{e} \rightarrow \bar{\nu}_{\mu}$ wrong-sign muon events. The distributions are shown for 


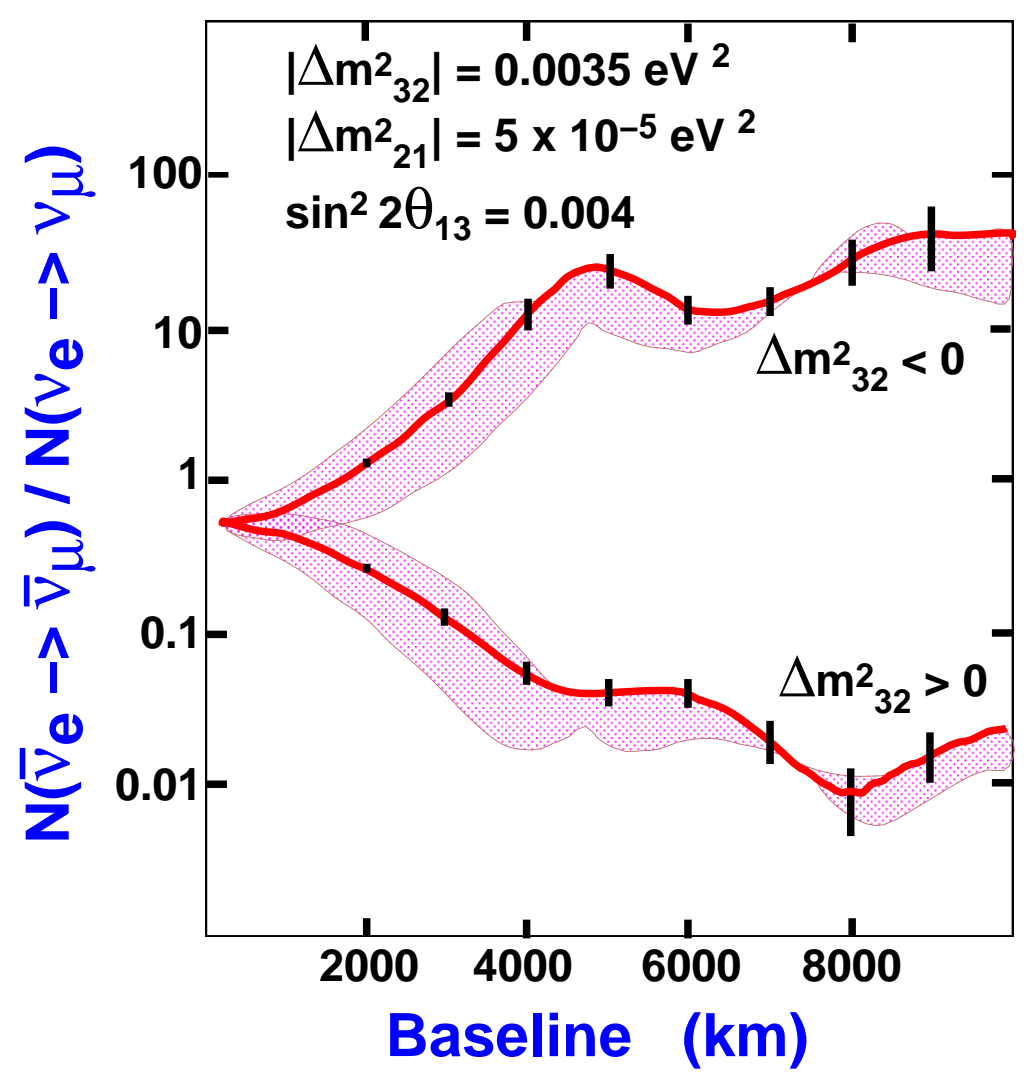

Figure 19: (Color) Predicted ratios of wrong-sign muon event rates when positive and negative muons are stored in a $20 \mathrm{GeV} \mathrm{NF}$, shown as a function of baseline. A muon measurement threshold of $4 \mathrm{GeV}$ is assumed. The lower and upper bands correspond, respectively, to the two possible neutrino mass eigenstate orderings, as labeled. The widths of the bands show how the predictions vary as the $C P$ violating phase $\delta$ is varied from $-\pi / 2$ to $+\pi / 2$, with the thick lines showing the predictions for $\delta=0$. The statistical error bars correspond to a NF yielding a data sample of $10^{21}$ decays with a 50 kton detector. Figure from Ref. [82].

a range of positive and negative values of $\Delta m_{32}^{2}$. Note that, after allowing for the factor of two difference between the neutrino and antineutrino cross sections, for a given $\left|\Delta m_{32}^{2}\right|$, if $\Delta m_{32}^{2}>0$ we would expect to observe a lower wrong-sign muon event rate and a harder associated spectrum when positive muons are stored in the NF than when negative muons are stored. On the other hand, if $\Delta m_{32}^{2}<0$ we would expect to observe a higher wrong-sign muon event rate and a softer associated spectrum when positive muons are stored in the NF than when negative muons are stored. Hence, measuring the differential spectra when positive and negative muons are alternately stored in the NF can both enable the sign of $\Delta m_{32}^{2}$ to be unambiguously determined [84, and also provide a measurement of $\Delta m_{32}^{2}$ and a consistency check between the behavior of the rates and energy distributions.

\subsubsection{Other Channels}

In practice, to measure $\theta_{13}$, determine the mass hierarchy, and search for $C P$ violation, the analysis of the wrong-sign muon rates must be performed allowing all of the oscillation parameters to simultaneously vary within their uncertainties. Since the relationship between the measured quantities and the underlying mixing parameters is complicated, with a minimal set of measurements it may not be possible to identify a unique region of parameter space consistent with the data. For the next generation of conventional neutrino beam (superbeam) experiments, a detailed discussion of this problem can be found in Refs. $[85 ;$ 86; 87; 88; 89; 90. To understand the nature of the challenge, Fig. 22 shows, 


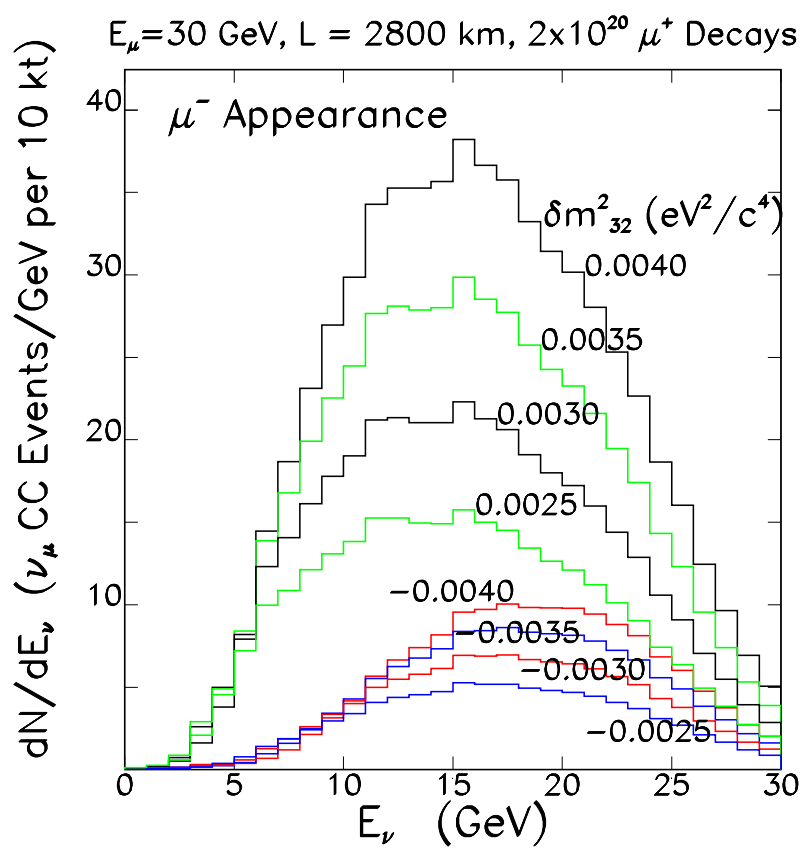

Figure 20: (Color) Predicted measured energy distributions for CC events tagged by a wrong-sign (negative) muon from $\nu_{e} \rightarrow \nu_{\mu}$ oscillations (no cuts or backgrounds), shown for various $\Delta m_{32}^{2}$, as labeled. The predictions correspond to $2 \times 10^{20}$ decays, $E_{\mu}=30 \mathrm{GeV}, L=2800 \mathrm{~km}$, and a representative set of values for $\Delta m_{12}^{2}, \sin ^{2} 2 \theta_{13}, \sin ^{2} 2 \theta_{23}, \sin ^{2} 2 \theta_{12}$, and $\delta$. Results are from Ref. [84].

as a function of $\theta_{13}, \theta_{23}, \delta$ and the assumed mass hierarchy, the predicted number of wrong-sign muon events when negative muons are stored in the NF, versus the corresponding rate when positive muons are stored. The ellipses show how the predicted rates vary as the $C P$ phase $\delta$ varies. All of the $C P$ conserving points $(\delta=0$ and $\pi$ ) lie on the diagonal lines. Varying the mixing angles moves the ellipses up and down the lines. Varying the mass hierarchy moves the family of ellipses from one diagonal line to the other. Note that the statistics are large, and the statistical errors would be barely visible if plotted on this figure. Given these statistical errors for the parameter region illustrated by the figure, determining the mass hierarchy (which diagonal line the measured point is closest to) will be straightforward. Determining whether there is $C P$ violation in the lepton sector will amount to determining whether the measured point is consistent with being on the $C P$ conserving line. Determining the exact values for the mixing angles and $\delta$ is more complicated, since various combinations can result in the same predicted values for the two measured rates. This is the origin of possible false solutions [91; 92$]$ in the three-flavor mixing parameter space. To eliminate those false solutions, event samples other than $\nu_{e} \rightarrow \nu_{\mu}$ transitions tagged by wrong-sign muons will be important. We have seen that, in the presence of $\nu_{e} \rightarrow \nu_{\mu}$ oscillations, the $\nu_{\mu}$ CC interactions produce negative muons (i.e., wrong-sign muons). Similarly, $\bar{\nu}_{\mu} \rightarrow \bar{\nu}_{e}$ oscillations produce wrong-sign electrons, $\bar{\nu}_{\mu} \rightarrow \bar{\nu}_{\tau}$ oscillations produce events tagged by a $\tau^{+}$, and $\nu_{e} \rightarrow \nu_{\tau}$ oscillations produce events tagged by a $\tau^{-}$. Hence, there is a variety of information that can be used to measure or constrain neutrino oscillations at a NF, namely the rates and energy distributions of events tagged by

(i) right-sign muons

(ii) wrong-sign muons

(iii) electrons or positrons (their charge is difficult to determine in a massive detector)

(iv) positive $\tau$-leptons 


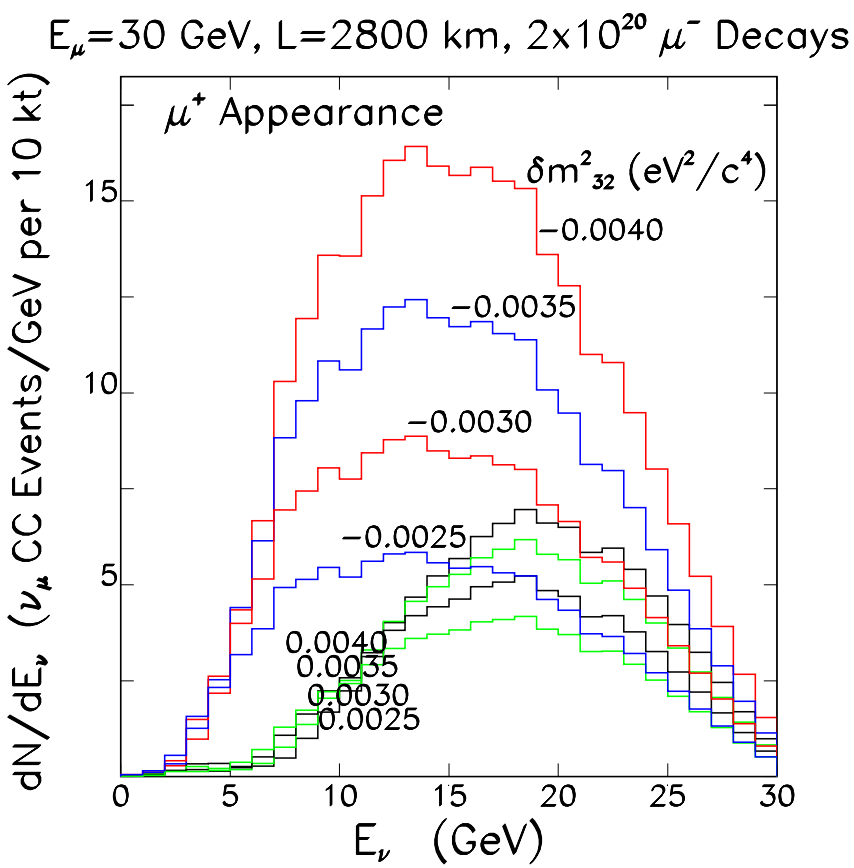

Figure 21: (Color) Same as in Fig. 20, for CC events tagged by a wrong-sign (positive) muon from $\bar{\nu}_{e} \rightarrow \bar{\nu}_{\mu}$ oscillations. Results are from Ref. [84].

$(v)$ negative $\tau$-leptons

(vi) no charged lepton.

If these measurements are made when there are alternately positive and negative muons decaying in the storage ring, there are a total of 12 spectra that can be used to extract information about the oscillations. Some examples of the predicted measured spectra are shown as a function of the oscillation parameters in Figs. 23 and 24 for a 10 kton detector sited $7400 \mathrm{~km}$ downstream of a $30 \mathrm{GeV}$ NF. These distributions are sensitive to the oscillation parameters, and can be fit simultaneously to extract the maximum information. Clearly, the high intensity $\nu_{e}, \bar{\nu}_{e}, \nu_{\mu}$, and $\bar{\nu}_{\mu}$ beams at a NF would provide a wealth of precision oscillation data. The full value of this wealth of information has not been completely explored, but some specific things to be noted are:

(i) It has been shown [58; 92; 93; 94] that the various measurements at a NF provide sufficient information to eliminate false solutions within the three-flavor parameter space. Indeed, the wealth of information in the NF data is essential for this purpose.

(ii) If $\sin ^{2} 2 \theta_{13}$ exceeds $\sim 0.001$ the $\nu_{e} \rightarrow \nu_{\tau}$ channel is particularly important, both as a means to suppress the false solutions $\left[91 ; 93 ; 94\right.$, and also as the only direct experimental probe of $\nu_{e} \leftrightarrow \nu_{\tau}$ transitions. The ability of the $\nu_{e} \rightarrow \nu_{\tau}$ measurements to eliminate false solutions is illustrated in Fig. 25, which, for a representative set of oscillation parameters, shows as a function of the $C P$ phase $\delta$, the location of the false solution with respect to the correct solution in $\theta_{13}$-space (or more precisely, the distance between the two solutions $\Delta \theta)$. Note that, when compared with the $\nu_{e} \rightarrow \nu_{\mu}$ case, $\Delta \theta$ has the opposite sign for $\nu_{e} \rightarrow \nu_{\tau}$. In practice, this means that together the two measurements enable the false solution to be effectively eliminated.

(iii) Within the three-flavor framework, the relationship between the measured oscillation probabilities and the associated oscillation parameters is complicated. Experimental redundancy, permitting the over-determination of the oscillation parameters, is likely to prove essential, both to weed out misleading measurements and to ensure that the three-flavor framework is correct. 


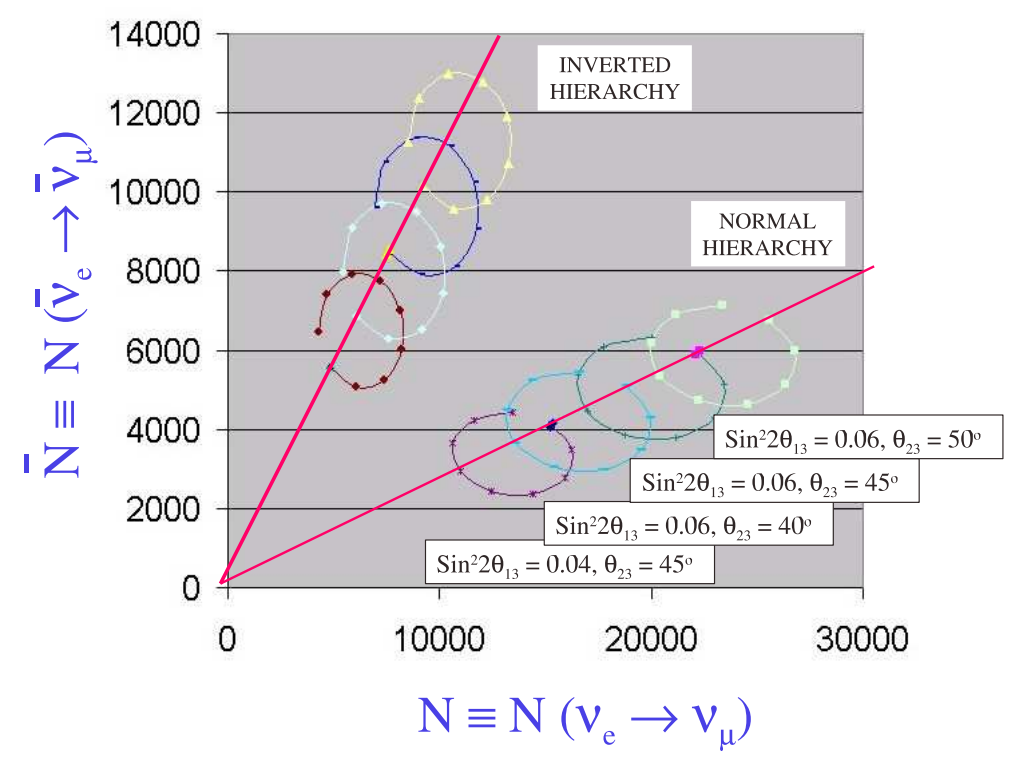

Figure 22: (Color) The predicted number of wrong-sign muon events when negative muons are stored in the NF, versus the corresponding rate when positive muons are stored, shown as a function of $\theta_{13}, \theta_{23}, \delta$ and the assumed mass hierarchy, as labeled. The calculation corresponds to a $16 \mathrm{GeV}$ NF with a baseline of $2000 \mathrm{~km}$, and 10 years of data taking with a 100 kton detector and $2 \times 10^{20} \mu^{+}$and $2 \times 10^{20} \mu^{-}$ decays in the beam-forming straight section per year. The ellipses show how the predicted rates vary as the $C P$ phase $\delta$ varies. Figure from Ref. [22].

\subsubsection{Neutrino Factory Calculations}

To understand how sensitive NF measurements will be in determining $\theta_{13}$ and the neutrino mass hierarchy, and searching for $C P$ violation in the lepton sector, we must consider the impact of statistical and systematic uncertainties, correlations between the parameters that vary within fits to the measured distributions, and the presence or absence of false solutions in the three-flavor mixing parameter space. To take account of these effects, and to see which different neutrino oscillation experiments best complement one another, a global fitting program has been created [92; 96] that uses simulated right-sign muon and wrong-sign muon data sets, and includes:

(i) Beam spectral and normalization uncertainties

(ii) Matter density variations of $5 \%$ about the average value

(iii) Constraint of solar neutrino oscillation parameters within the post-KamLAND LMA region

(iv) Simulation of $\nu_{\mu} \mathrm{CC} \mathrm{QE}, \nu_{\mu}$ and $\nu_{e} \mathrm{CC}$ inelastic, and NC events for all flavors; note that the $\mathrm{NC}$ events are included in the analysis as a source of background, but the NC signal is not yet exploited as an additional constraint

(v) A check of the influence of cross section uncertainties (this mostly affects energies lower than those of interest for Neutrino Factories)

(vi) Energy-dependent detection efficiencies, enabling energy threshold effects to be taken into account

(vii) Gaussian energy resolutions 

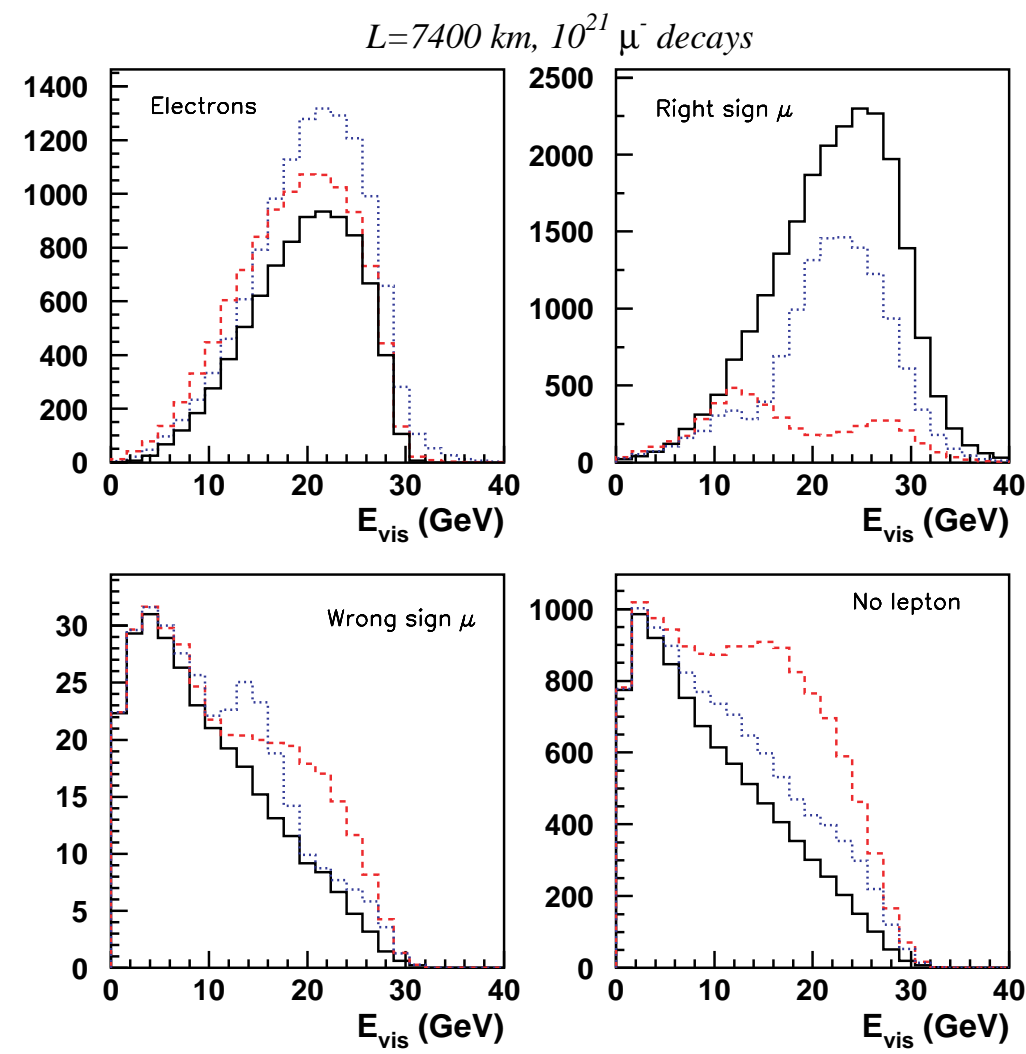

Figure 23: (Color) Visible energy spectra for four event classes when $10^{21} \mu^{-}$decay in a $30 \mathrm{GeV} \mathrm{NF}$ at $L=7400 \mathrm{~km}$. Black solid histogram: no oscillations. Blue dotted histogram: $\Delta m_{32}^{2}=3.5 \times 10^{-3} \mathrm{eV}^{2} / \mathrm{c}^{4}$, $\sin ^{2} \theta_{23}=1$. Red dashed histogram: $\Delta m_{32}^{2}=7 \times 10^{-3} \mathrm{eV}^{2} / \mathrm{c}^{4}, \sin ^{2} \theta_{23}=1$. The distributions are for an ICANOE-type detector, and are from Ref. [95].

(viii) Flavor, charge, and event misidentification

(ix) Overall energy-scale and normalization errors

(x) An analysis of statistical and systematic precisions, and the ability to eliminate false solutions

The global fitting program has been used to calculate the expected $\sin ^{2} 2 \theta_{13}$ discovery reaches for the next generation of oscillation experiments, where the discovery reach is defined as the minimum value of $\sin ^{2} 2 \theta_{13}$ for which $\theta_{13}=0$ can be excluded with a significance of $3 \sigma$. Based on these calculations, the anticipated sensitivity is expected to improve by about an order of magnitude over the next decade. We therefore anticipate that before the decision is taken to construct a NF we will already know whether $\sin ^{2} 2 \theta_{13}>\mathcal{O}\left(10^{-2}\right)$. The calculated sensitivity at a NF is shown in Fig. 26, which also shows the sensitivities for determining the mass hierarchy and observing $C P$ violation. More explicitly, the figure shows the minimum value of $\sin ^{2} 2 \theta_{13}$ for which three experimental goals could be achieved: $(i)$ the observation of a non-zero value of $\theta_{13},(i i)$ the determination of the neutrino mass hierarchy, and (iii) the observation of non-zero $C P$ violation in the lepton sector if the underlying $\delta$ corresponds to maximal $C P$ violation. The three groups of bars correspond to three different experimental scenarios, with different baselines. The favored scenario is the one illustrated by the bottom group of three bars, for which there are two detectors, one at $L=7500 \mathrm{~km}$ and the other at $L=3000 \mathrm{~km}$. Note that, at a NF, $\sin ^{2} 2 \theta_{13}$ can be measured, the neutrino mass hierarchy can be determined, and a search for $C P$ violation in the lepton sector can be made for all values of $\sin ^{2} 2 \theta_{13}$ down to $\mathcal{O}\left(10^{-4}\right)$, or even a little less. 

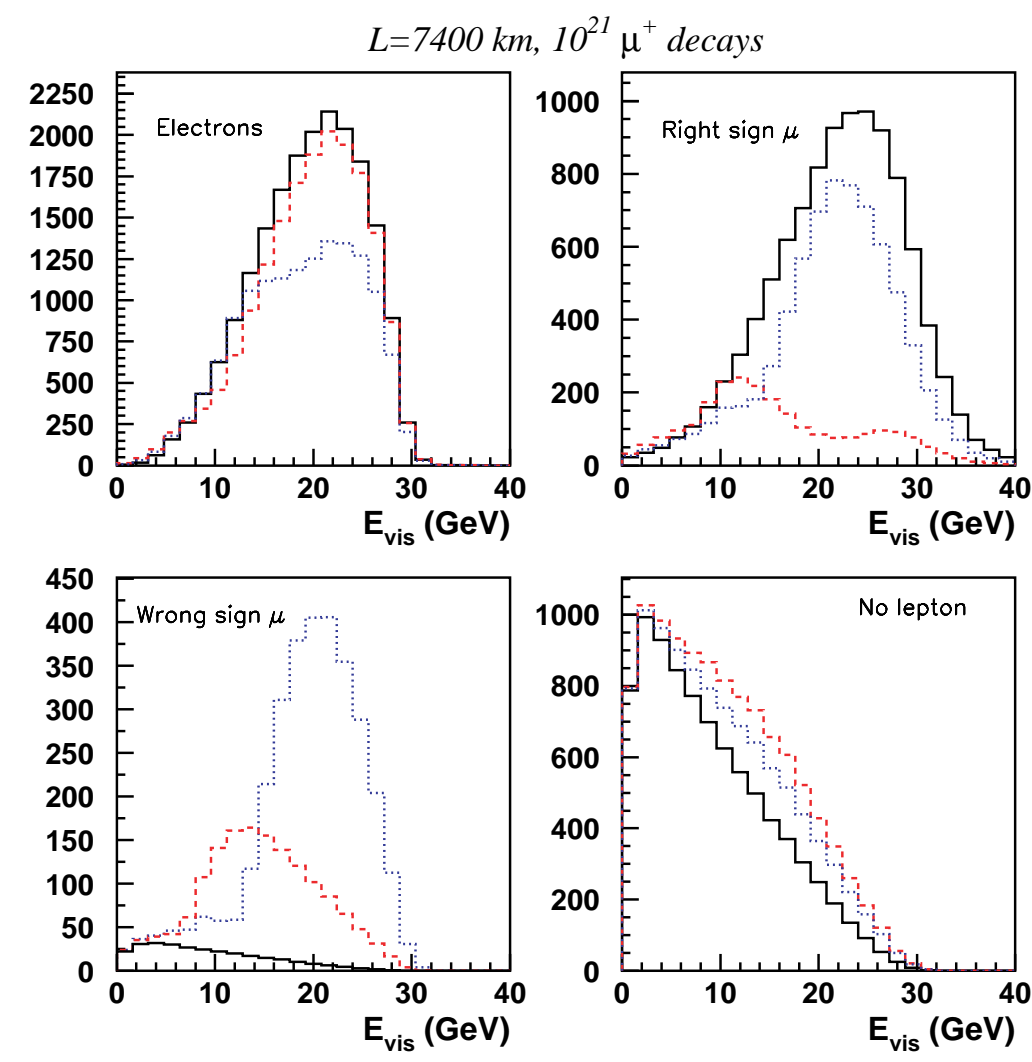

Figure 24: (Color) Same as in Fig. 23, but with positive muons circulating in the storage ring. The difference between the two figures is due to the different cross section for neutrinos and antineutrinos, and to matter effects. The distributions are for an ICANOE-type detector, and are from Ref. [95].

If $\sin ^{2} 2 \theta_{13}$ is fairly large, superbeam experiments may also establish its value, and perhaps determine the mass hierarchy and begin the search for $C P$ violation. Figure 27 illustrates the role of a NF over a broad range of $\sin ^{2} 2 \theta_{13}$ values. The figure shows, as a function of the underlying value of $\sin ^{2} 2 \theta_{13}$, the $1 \sigma$ precision on the determination of the phase $\delta$ at a $\mathrm{NF}$, and at a representative high-performance superbeam, together with the combined NF plus superbeam sensitivity. Below values of $\sin ^{2} 2 \theta_{13} \sim 0.01$ the NF sensitivity is significantly better than the sensitivity that can be achieved with superbeams, and indeed provides the only sensitivity to the $C P$ phase if $\sin ^{2} 2 \theta_{13}$ is significantly smaller than 0.01 . Above $\sin ^{2} 2 \theta_{13} \sim 0.02$ the NF measurements still enable a modest improvement to the $C P$ violation measurement sensitivity. In this case the uncertainty on the matter density, which is believed to be $\mathcal{O}(5 \%)$, is likely to be a limiting uncertainty for $C P$ violation measurements [98]. Improved knowledge of the matter density along the neutrino flight-path would improve the expected NF sensitivity for $\sin ^{2} 2 \theta_{13}>0.02$. In addition, Bueno et al. [95] have shown that the energy dependencies of matter and $C P$ violating effects are different, and can be exploited to further separate the two effects.

The prospects for determining the neutrino mass hierarchy and discovering $C P$ violation depend not only on the value of $\theta_{13}$, but also on $\delta$. Figure 28 shows, as a function of $\sin ^{2} 2 \theta_{13}$, for various combinations of experiments, the fraction of all possible values of $\delta$ for which the mass hierarchy can be determined (left panel) and $C P$ violation can be discovered (right panel). The greatly improved sensitivity obtained from a NF for small values of $\theta_{13}$ is evident.

Finally, to appreciate the exquisite sensitivity that would be made possible by a NF, it is interesting to consider what would happen if $\sin ^{2} 2 \theta_{13}=0$. The case $\theta_{13}=0$ is very special. The number of mixing angles needed to describe the $3 \times 3$ unitary neutrino mixing matrix would be reduced from three to 


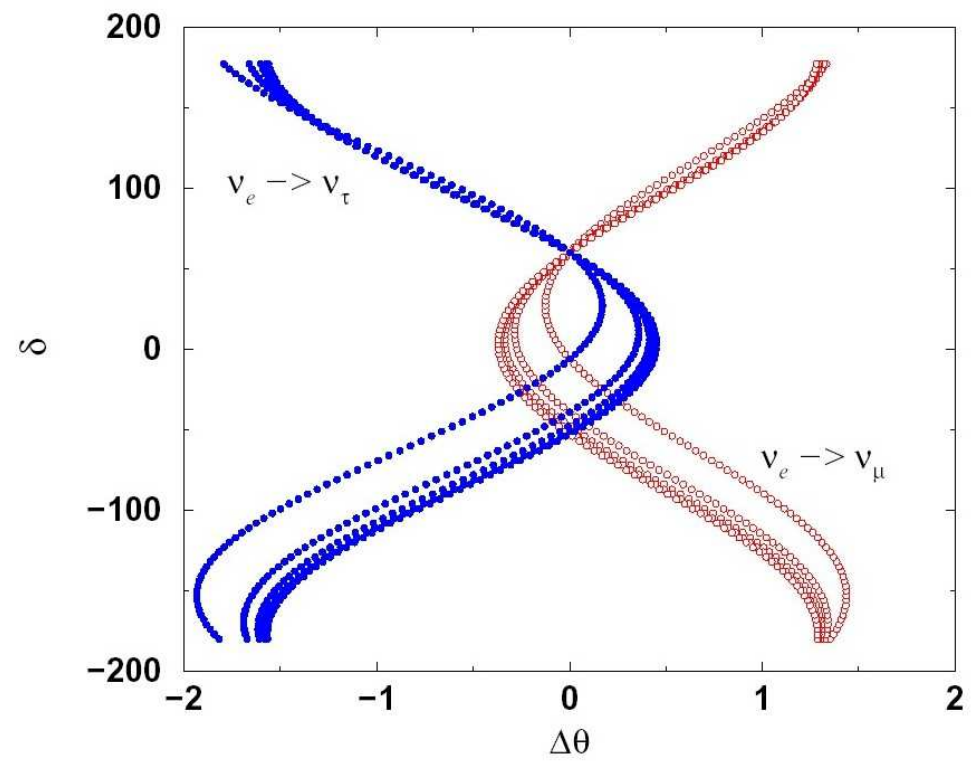

Figure 25: (Color) Equi-probability curves in the $(\Delta \theta, \delta)$ plane, for $\bar{\theta}_{13}=5^{\circ}, \bar{\delta}=60^{\circ}, E_{\nu} \in[5,50] \mathrm{GeV}$ and $L=732 \mathrm{~km}$ for the $\nu_{e} \rightarrow \nu_{\mu}$ and $\nu_{e} \rightarrow \nu_{\tau}$ oscillations. $\Delta \theta$ is defined as the difference between the reconstructed parameter $\theta_{13}$ and the input parameter $\bar{\theta}_{13}$, i.e., $\Delta \theta=\theta_{13}-\bar{\theta}_{13}$. The four subcurves are for different neutrino energies between $5 \mathrm{GeV}$ and $45 \mathrm{GeV}$. From Ref. [93].

Table 10: Summary of the various experiments that are discussed in the text and Figs. 27 and 28. Table from Ref. [99].

\begin{tabular}{lccc}
\hline Experiment & $\begin{array}{c}\text { Detector mass } \\
(\text { kton })\end{array}$ & $\begin{array}{c}\text { Proton power } \\
(\mathrm{MW})\end{array}$ & $\begin{array}{c}\text { Running time } \\
\text { (years) }\end{array}$ \\
\hline \hline $\mathrm{NO} \nu \mathrm{A}$ & 30 & 0.4 & $3 \nu+3 \bar{\nu}$ \\
$\mathrm{FPD}+\mathrm{NO} \nu \mathrm{A}$ & 30 & 2.0 & $3 \nu+3 \bar{\nu}$ \\
$\mathrm{FPD}+\mathrm{NO} \nu \mathrm{A}+2^{\text {nd }} \mathrm{NO} \nu \mathrm{A}$ & $30(+30)$ & 2 & $6(3) \nu+6(3) \bar{\nu}$ \\
$\mathrm{FPD}+\mathrm{New}$ Long Baseline & 125 or 500 & $2+2$ & $5 \nu+5 \bar{\nu}$ \\
$\mathrm{T} 2 \mathrm{~K}$ & 50 & 0.77 & $3 \nu+3 \bar{\nu}$ \\
$\mathrm{T} 2 \mathrm{~K}^{*}$ & 50 & 4 & $3 \nu+3 \bar{\nu}$ \\
$\mathrm{T} 2 \mathrm{HK}$ & 500 & 4 & $3 \nu+3 \bar{\nu}$ \\
$3000 \mathrm{~km} \mathrm{NF}$ & 50 & 4 & $3 \nu+3 \bar{\nu}$ \\
\hline
\end{tabular}

two, suggesting the existence of a new conservation law resulting in an additional constraint on the elements of the mixing matrix. The discovery of a new conservation law happens rarely in physics, and almost always leads to revolutionary insights in our understanding of how the physical universe works. Hence, if it were possible to establish that $\theta_{13}=0$, it would be a major discovery. Note that in the limit $\theta_{13} \rightarrow 0$, the oscillation probability for $\nu_{e} \leftrightarrow \nu_{\mu}$ transitions is non-zero, and is given by:

$$
P\left(\nu_{e} \rightarrow \nu_{\mu}\right)=\frac{\Delta m_{21}^{2}}{\Delta m_{31}^{2}} \sin ^{2} 2 \theta_{12} \cos ^{2} \theta_{23} \frac{\sin ^{2} A \Delta}{A^{2}},
$$

where the matter parameter $A=1$ if the neutrino energy corresponds to the matter resonance, which for a long-baseline terrestrial experiment means neutrino energies $E \sim 12 \mathrm{GeV}$. In addition, if the baseline $L$ is chosen such that $L / E$ corresponds to the oscillation maximum, then $\sin ^{2} \Delta=1$, and we 


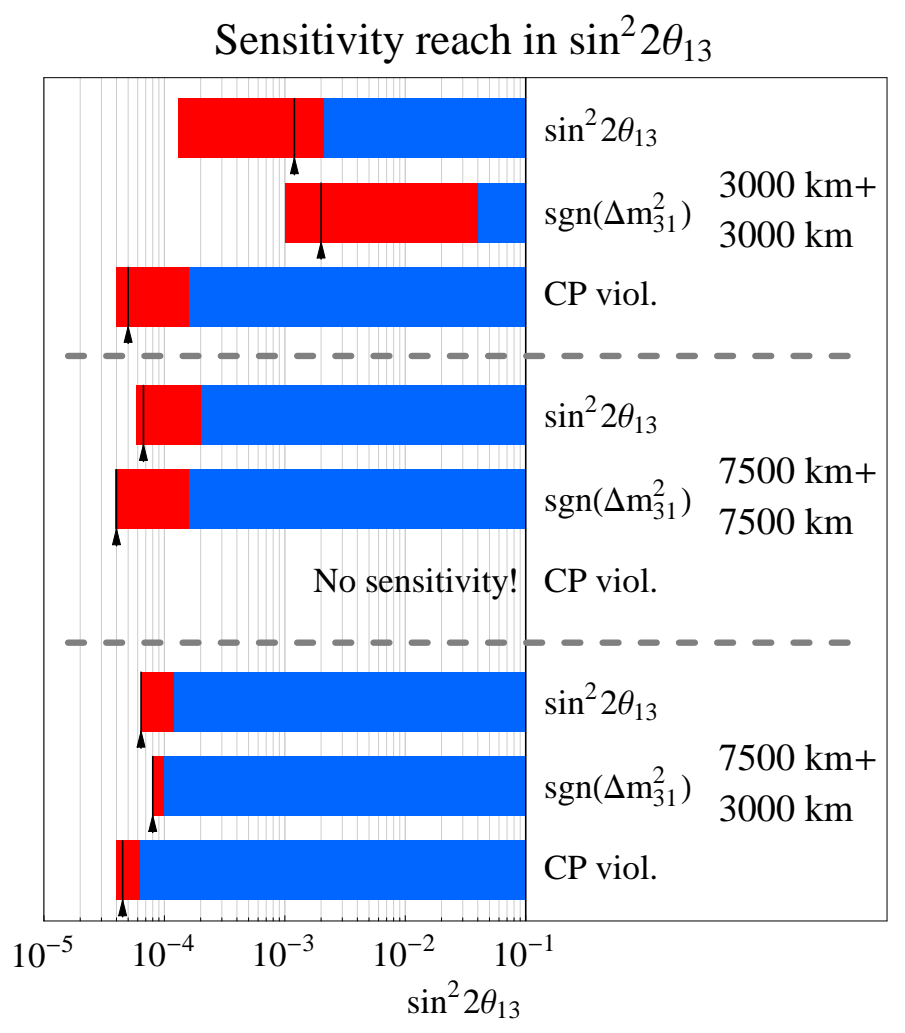

Figure 26: (Color) The sensitivity reaches as a function of $\sin ^{2} 2 \theta_{13}$ for $\sin ^{2} 2 \theta_{13}$ itself, the sign of $\Delta m_{31}^{2}>0$, and (maximal) $C P$ violation $\delta_{\mathrm{CP}}=\pi / 2$ for each of the indicated baseline combinations. The bars show the ranges in $\sin ^{2} 2 \theta_{13}$ where sensitivity to the corresponding quantity can be achieved at the $3 \sigma$ confidence level. The dark bars mark the variations in the sensitivity limits by allowing the true value of $\Delta m_{21}^{2}$ to vary in the $3 \sigma$ LMA-allowed range given in Ref. [97] and others $\left(\Delta m_{21}^{2} \sim\right.$ $\left.4 \times 10^{-5} \mathrm{eV}^{2}-3 \times 10^{-4} \mathrm{eV}^{2}\right)$. The arrows/lines correspond to the LMA best-fit value. Figure from Ref. [58].

have:

$$
P\left(\nu_{e} \rightarrow \nu_{\mu}\right) \sim \sin ^{2} 2 \theta_{12} \cos ^{2} \theta_{23} \frac{\Delta m_{21}^{2}}{\Delta m_{31}^{2}}
$$

Substituting into this expression values for the oscillation parameters that are consistent with the present solar and atmospheric neutrino data, we are led to conclude that even if $\theta_{13}=0$, provided the neutrino energy and baseline are chosen appropriately, $\nu_{e} \leftrightarrow \nu_{\mu}$ transitions are still directly observable in an appearance experiment if oscillation probabilities of $\mathcal{O}\left(10^{-4}\right)$ are observable. Hence, if $\theta_{13}$ is very small, the ideal neutrino oscillation experiment will be a long baseline experiment that uses neutrinos with energies close to $12 \mathrm{GeV}$, i.e., uses a baseline such that $L / E$ corresponds to the oscillation maximum, and is sensitive to values of $P\left(\nu_{e} \leftrightarrow \nu_{\mu}\right) \sim 10^{-4}$ or smaller. Neutrino Factories provide the only way we know to satisfy these experimental requirements.

\section{Machine Design}

In this Section we discuss design details for a particular design, the Study IIa linear configuration described in Refs. [22; 23]. Other, equivalent, designs of this type differ in their specific design choices, but the overall aspects of the design discussed here are representative of the present state of NF design. 


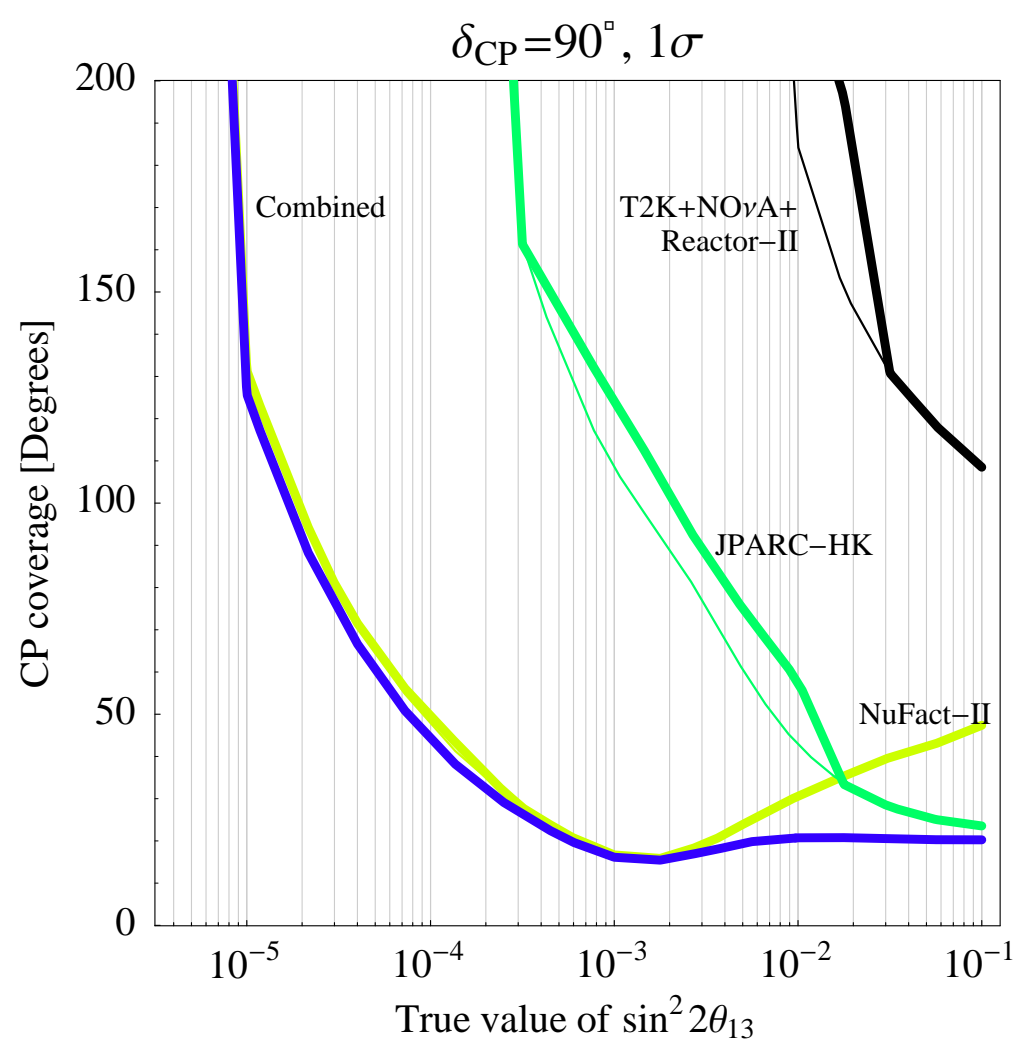

Figure 27: (Color) The $1 \sigma$ precision on the determination of the phase $\delta$ at a NF, and at a representative high-performance superbeam, together with the combined NF plus superbeam sensitivity. The sensitivities are shown as a function of the underlying value of $\sin ^{2} 2 \theta_{13}$. The thin curves correspond to cases where the the sign-degeneracy is not taken into account. Calculation from the authors of Ref. [92].

\subsection{Front End}

In Study II [21], which included the most detailed NF cost estimate to date, it was found that the front end of the NF (that part of the facility between the target and the first linear accelerator) accounts for a large fraction of the total facility costs. However, several recent developments [22; 23] have shown that a new, significantly less expensive, design for the front end is possible:

- A new approach to bunching and phase rotation based on adiabatic rf bunching [100; 101; 102; 103; 104] was developed; this avoids the expensive induction linacs used in the Study II design

- For a moderate cost, it was possible to double the transverse acceptance of the accelerator chain compared with its Study II design value

- The resulting diminished demands on transverse ionization cooling allow the design of a simplified cooling section with fewer components and reduced magnetic field strength

These considerations led to the Study IIa design. Monte Carlo simulations of the design made use of the code ICOOL [105]. The Study II and IIa buncher concepts are compared in Fig. 29, assuming the same longitudinal phase space after the target. Initially, there is a very large energy spread but only a small spread in time. In either case, the target is followed by a drift space that produces a strong correlation between time and energy. In the Study II design, the energy spread in the correlated beam was first flattened using a series of induction linacs. Although the induction linacs do an excellent job of phase rotation (reducing the final rms energy spread to 4.4\%), they are expensive and they permit only a single muon charge to be transmitted. The beam was then sent through a series of rf cavities 

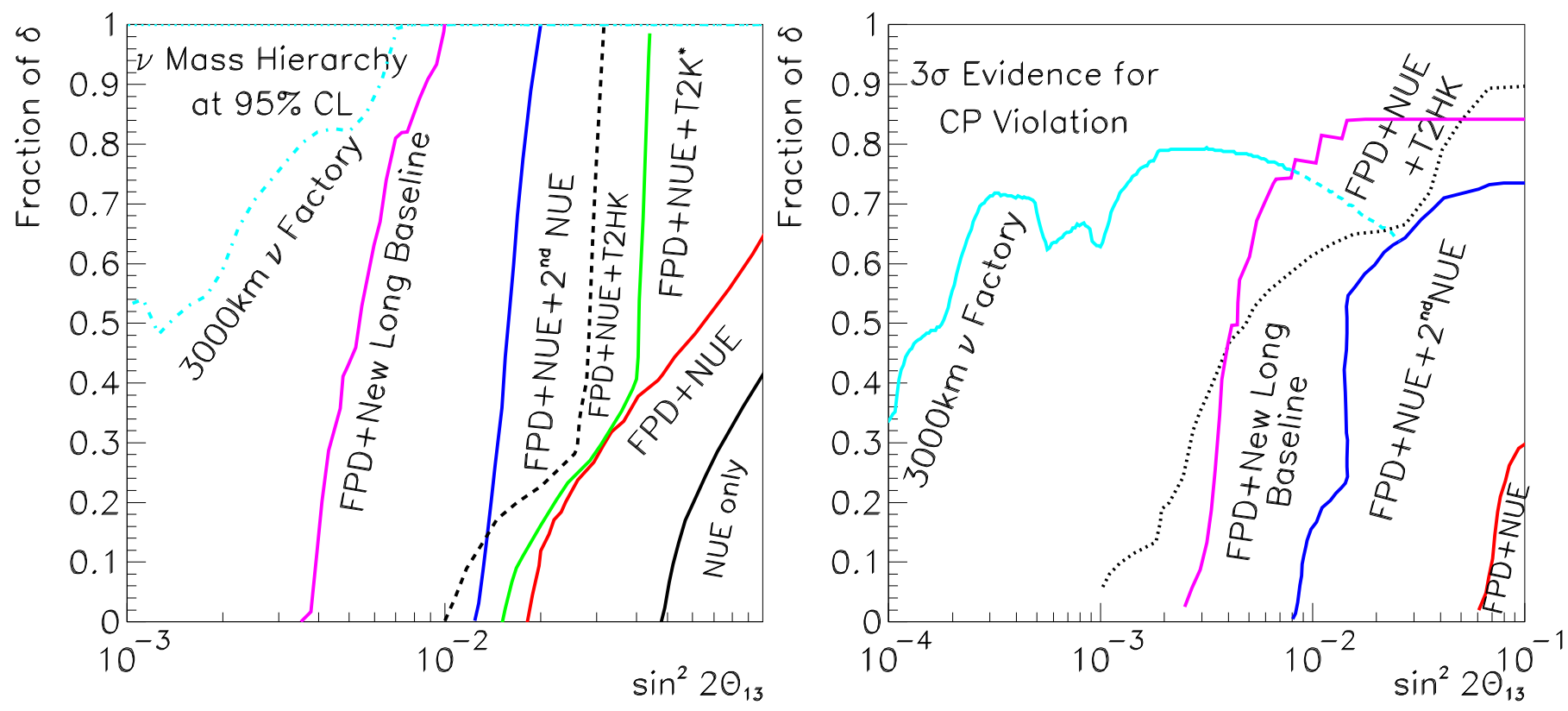

Figure 28: Regions of parameter space where the mass hierarchy (left) and $C P$ violation (right) can be observed at $95 \% \mathrm{CL}$ and at $3 \sigma$, respectively. $\mathrm{NO} \nu \mathrm{A}, \mathrm{T} 2 \mathrm{~K}$, etc. are defined in Table 10 .

for bunching, which resulted in an energy spread of $\approx 8 \%$. In the new Study IIa scheme, the correlated beam is first adiabatically bunched using a series of $\mathrm{rf}$ cavities with decreasing frequencies and increasing gradients. The beam is then phase rotated with a second string of rf cavities with decreasing frequencies and constant gradient. The final rms energy spread in the new design, $10.5 \%$, is worse than that for Study II, but still adequate for the Study IIa cooling channel.

The layout of the new 295-m-long front-end design is as follows: The first $\approx 12 \mathrm{~m}$ is used to capture pions produced in the target. The field is tapered smoothly from $20 \mathrm{~T}$ at the target to $1.75 \mathrm{~T}$ at the start of the drift section. As the field is tapered, the radial aperture of the beam pipe in this region increases from $7.5 \mathrm{~cm}$ at the target to $25 \mathrm{~cm}$. Next is a drift of $\approx 100 \mathrm{~m}$ where the pions decay into muons and where the energy-time correlation develops. The next $\approx 50 \mathrm{~m}$ is used for adiabatic bunching, followed by a phase rotation section that is also $\approx 50 \mathrm{~m}$ long. This leads to an ionization cooling channel some $80 \mathrm{~m}$ in length. The magnetic field is maintained at $1.75 \mathrm{~T}$, with very little ripple, over the decay, buncher, and rotator regions before changing adiabatically to the alternating field used in the cooling channel.

Initial beam distributions used in the simulations discussed here were generated using MARS [34]. The results are based on a $24 \mathrm{GeV}$ proton beam interacting with a Hg-jet target [106]. To minimize reabsorption of the pions, and to permit the beam dump to be conveniently located, the jet is incident at an angle of $100 \mathrm{mrad}$ to the solenoid axis, while the beam is incident at an angle of $67 \mathrm{mrad}$ to the solenoid axis. An independent study (see Study II [21]) showed that the resulting 33 mrad crossing angle gives near-peak acceptance for the produced pions. Particles that were propagated to the end of the front-end channel have a peak initial longitudinal momentum of $\approx 300 \mathrm{MeV} / \mathrm{c}$ with a long high-energy tail, and a peak initial transverse momentum of $\approx 180 \mathrm{MeV} / \mathrm{c}$. Optimizing the axial field profile in the capture region increased the final number of muons per proton in the accelerator acceptance by $\approx 10 \%$ compared with the design in Ref. [21].

In such a high radiation environment, it is important to evaluate the radiation dose being absorbed by the superconducting magnets in the collection region. Calculations with MARS indicate a peak deposition in the superconducting coils of $\approx 1 \mathrm{Mgy} / \mathrm{yr}$ for a $1 \mathrm{MW}$ beam running for an operational 

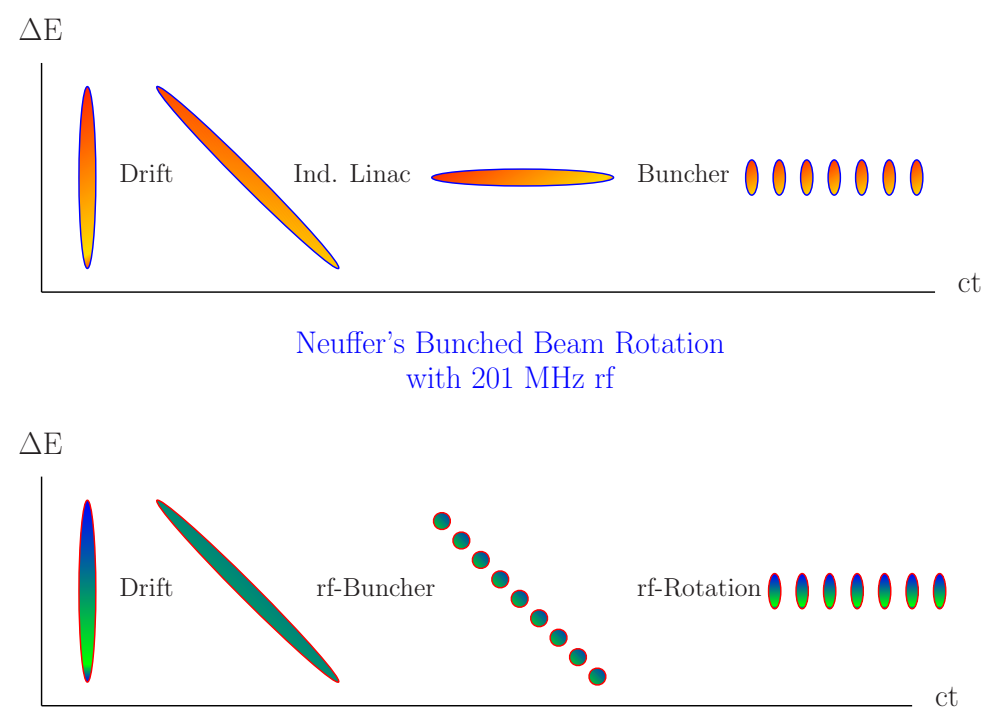

Figure 29: (Color) Comparison of the buncher concept used in Study Ila (lower plot) with the bunching system used in Study II (upper plot).

year of $10^{7} \mathrm{~s}$, compared with a lifetime dose for the insulation of $100 \mathrm{Mgy}$. Thus, the coil lifetime will be acceptable even at a $4 \mathrm{MW}$ beam power.

The buncher lattice employs a 75-cm cell length, including a 50-cm-long rf cavity. A 50-cm-long solenoid placed outside the rf cavity provides a uniform field to minimize beam losses from momentum stop-bands. The buncher design in Ref. [23] contains 27 cavities with 13 discrete frequencies (decreasing from 333 to $234 \mathrm{MHz}$ ) and gradients varying from 5 to $10 \mathrm{MV} / \mathrm{m}$. To optimize the design, fewer cavities are used at the beginning where the required gradients are small.

The phase rotation cells are very similar to the buncher cells, the major difference being the use of tapered Be windows on the cavities because of the higher rf gradient. The phase rotation section employs 72 cavities with 15 different frequencies, decreasing from 232 to $201 \mathrm{MHz}$, at a fixed gradient of $12.5 \mathrm{MV} / \mathrm{m}$.

One cell of the cooling channel is shown in Fig. 30, taken from Ref. [23]. The channel has a relatively flat transverse beta function with a magnitude of about $80 \mathrm{~cm}$. Most of the $150 \mathrm{~cm}$ cell length is taken

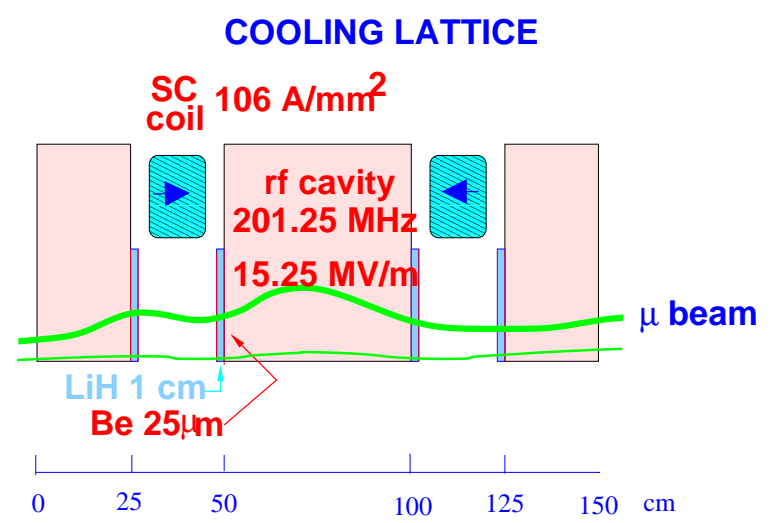

Figure 30: (Color) Schematic of one cell of the cooling section used in Study IIa. 
up by the 50-cm-long rf cavities. The cavities have a frequency of $201.25 \mathrm{MHz}$ and a gradient of $15.25 \mathrm{MV} / \mathrm{m}$. A novel aspect of this new cooling channel design comes from using the windows on the rf cavity as the cooling absorbers. (Because the beta function is nearly constant, emittance heating is not significantly worse at the rf window locations than at other locations.) The window comprises a 1-cm-thick LiH energy absorber with $25 \mu \mathrm{m}$ thick Be coatings. (The Be will, in turn, have a thin coating of TiN to prevent multipactoring [107].) The channel employs one solenoid per half-cell, located between the rf cavities, to provide an alternating $2.8 \mathrm{~T}$ solenoidal field.

The left plot of Fig. 31 shows the calculated reduction in normalized transverse emittance along the cooling channel, and the right plot shows the normalized longitudinal emittance. The channel provides more than a factor of two reduction from the initial emittance, resulting in an output emittance of $\epsilon_{T}=7.1 \mathrm{~mm} \mathrm{rad}$, and an increase in transmission through the downstream acceleration system by a factor of 1.7 .

As a figure-of-merit for the cooling channel, Fig. 32 shows the number of muons per 24-GeV proton that fit into the accelerator transverse normalized acceptance of $A_{T}=30 \mathrm{~mm}$ rad and normalized longitudinal acceptance of $A_{L}=150 \mathrm{~mm}$. The calculated value is $0.170 \pm 0.006$ muons per primary proton - the same as that obtained with the Study II design. We see that the updated Study IIa design maintains the performance at the entrance to the acceleration section with a significantly simpler, shorter, and thus less expensive design. As noted earlier, the Study IIa front end transmits both signs of muon produced in the decay channel. With appropriate modifications to the transport lines going into the storage ring, both positive and negative muons can be injected for each incoming primary proton bunch. To make use of this feature, the injection timing must be adjusted such that decays from $\mu^{-}$and $\mu^{+}$in the beam-forming straight section are separated in time. Figure 33 shows the longitudinal phase space population within a single bunch.

\subsection{Acceleration System}

As the final momentum of the muon beam is a few tens of $\mathrm{GeV} / \mathrm{c}$ and the capture, decay, and cooling processes take place at a momentum of roughly $0.2 \mathrm{GeV} / \mathrm{c}$, a means to rapidly accelerate the beam is needed. Table 11 gives the design parameters of the Study IIa acceleration system. Transverse acceptance, $A_{\perp}$, is defined such that the maximum displacement of a transmitted particle from the reference orbit is $\sqrt{\beta_{\perp} A_{\perp} m c / p}$, where $p$ is the particle's total momentum, $m$ is its rest mass, $\beta_{\perp}$ is the beta function, and $c$ is the speed of light. A key feature of recent designs is the ability to accelerate bunch trains of both signs simultaneously. Figure 34 shows a compact layout for all of the acceleration systems considered in Study IIa [22; 23].
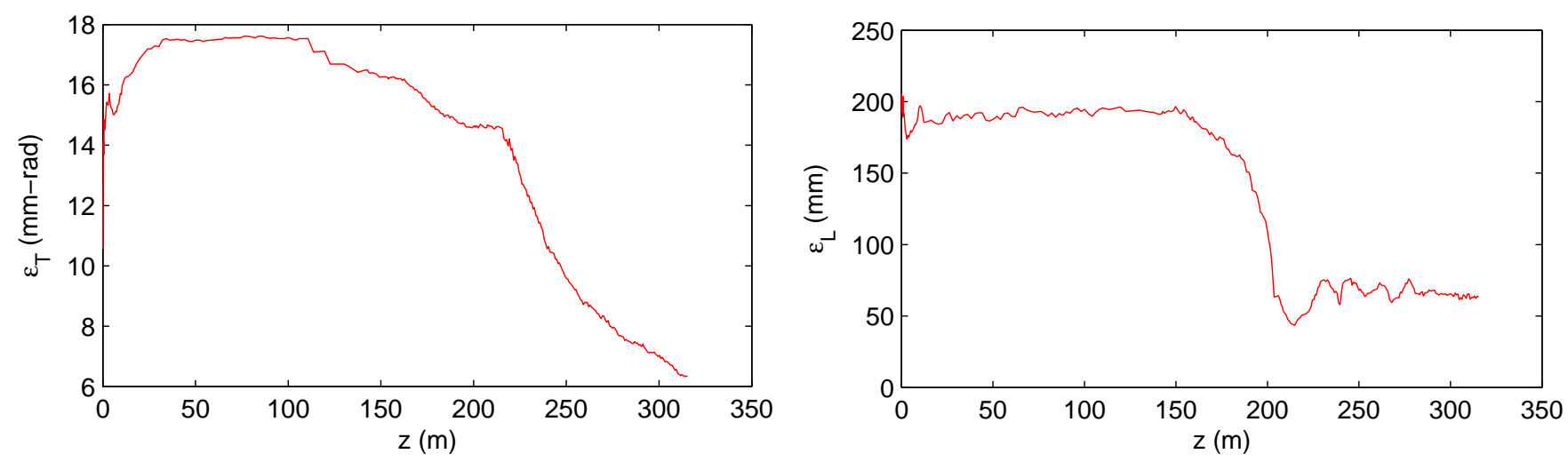

Figure 31: (Color) Normalized transverse emittance (left) and longitudinal emittance (right) along the front end for a momentum cut $0.1 \leq p \leq 0.3 \mathrm{GeV} / \mathrm{c}[23]$. 


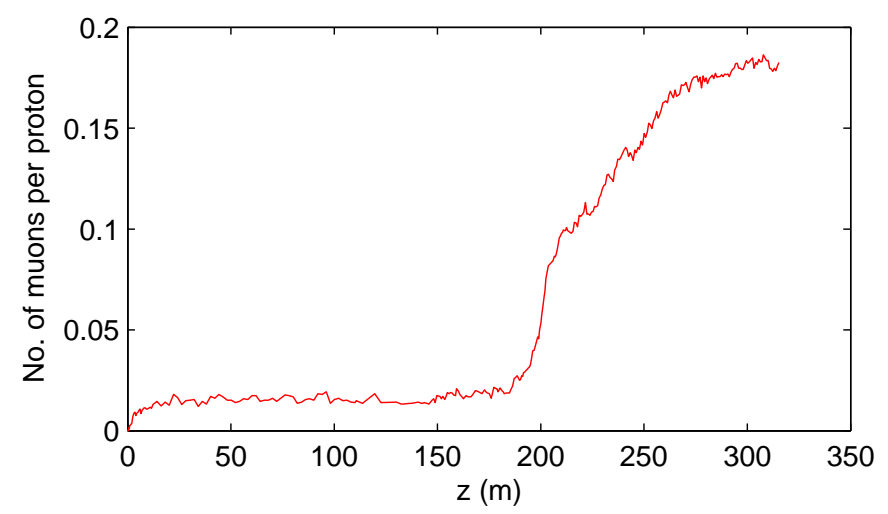

Figure 32: (Color) The number of muons per proton into an acceleration system transverse normalized acceptance of $A_{T}=30 \mathrm{~mm}$ rad and normalized longitudinal acceptance of $A_{L}=150 \mathrm{~mm}$ for a momentum cut $0.1 \leq p \leq 0.3 \mathrm{GeV} / \mathrm{c}[23]$.

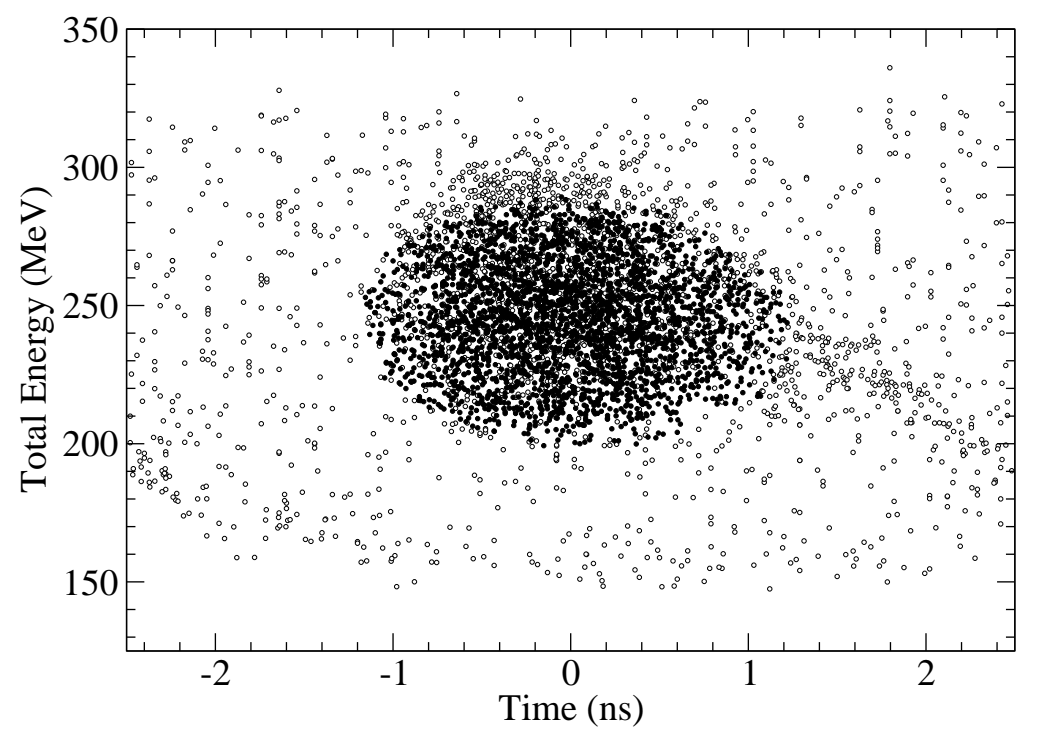

Figure 33: Longitudinal phase space of one bunch in the train at the end of the cooling section used in [23]. The open circles are all the particles that reach the end of the channel and the filled circles are particles within the accelerator transverse normalized acceptance of $A_{T}=30 \mathrm{~mm}$ rad and normalized longitudinal acceptance of $A_{L}=150 \mathrm{~mm}$ for a momentum cut $0.1 \leq p \leq 0.3 \mathrm{GeV} / \mathrm{c}$.

\subsubsection{FFAG Parameters}

In the Study IIa design, two factor-of-two FFAG stages are used: one to accelerate from 5 to $10 \mathrm{GeV}$, the other to accelerate from 10 to $20 \mathrm{GeV}$. Triplet lattices were chosen because they have been extensively studied and yield good longitudinal performance. The parameters that were adopted in Study IIa [22; 23$]$ are given in Table 12 .

The 201.25 MHz cavities are single-cell superconducting cavities, a choice dictated by the higher duty factor needed for acceleration. This choice is possible here - in contrast to the cavities in the cooling section-because these cavities are not immersed in a strong solenoidal field. Care was taken to design the lattice such that cavities can be located sufficiently far from nearby magnets to give acceptably low fields. The chosen energy gain per rf cavity is based on gradients already achieved in cavity studies at Cornell, namely $10 \mathrm{MV} / \mathrm{m}$ at $4.2 \mathrm{~K}$ (they have achieved $11 \mathrm{MV} / \mathrm{m}$ at $2.5 \mathrm{~K}$ ) [108]. This is a very conservative approach, as it is likely that improved fabrication and processing techniques will allow such cavities to achieve a gradient of $15 \mathrm{MV} / \mathrm{m}$ or higher. Energy gain and stored energy are 
Table 11: Acceleration system requirements used in Refs. [22; 23].

\begin{tabular}{lc}
\hline Initial kinetic energy $(\mathrm{MeV})$ & 187 \\
Final total energy $(\mathrm{GeV})$ & 20 \\
Normalized transverse acceptance (mm rad) & 30 \\
Normalized longitudinal acceptance $(\mathrm{mm})$ & 150 \\
Bunching frequency $(\mathrm{MHz})$ & 201.25 \\
Maximum muons per bunch & $1.1 \times 10^{11}$ \\
Muons per bunch train per sign & $3.0 \times 10^{12}$ \\
Bunches in train & 89 \\
Average repetition rate $(\mathrm{Hz})$ & 15 \\
Minimum time between pulses $(\mathrm{ms})$ & 20 \\
\hline
\end{tabular}

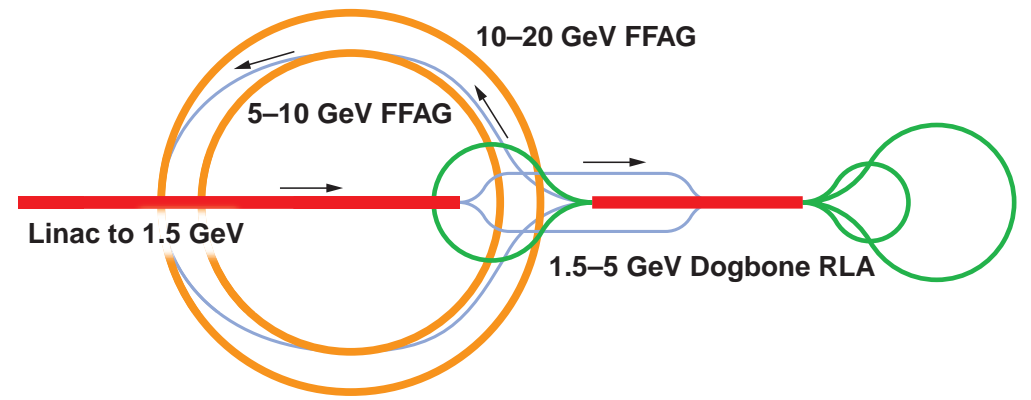

Figure 34: (Color) Potential layout for the acceleration systems.

computed by scaling from the values for the 300-mm-aperture Study II cavities [21; 108].

At the beam intensity given in Table 11, and with both signs of muon, about $16 \%$ of the stored energy will be extracted from the cavities in the 5-10 GeV FFAG, and about 27\% will be extracted in the 10-20 GeV FFAG. To maintain an average voltage of $7.5 \mathrm{MV}$ per cavity, the initial voltage must be increased to 7.8 MV for the 5-10 GeV FFAG and to 8.1 MV for the 10-20 GeV FFAG. The most important effect is a differential acceleration between the head and tail of the bunch train, which is about $1 \%$ for both cases. This should be partially correctable, if needed, by a phase offset between the cavity and the bunch train. At higher muon intensities corresponding to a $4 \mathrm{MW}$ proton driver, these beam-loading effects become more severe, but are still considered to be manageable.

\subsubsection{Low-Energy Acceleration}

A linac is used from the cooling channel energy to $1.5 \mathrm{GeV}$, followed by a "dogbone" RLA. To obtain a linac acceptance that can accommodate the necessary transverse emittance at the exit of the cooling channel, the lengths of the linac cryomodules must be kept short; Table 13 summarizes parameters for

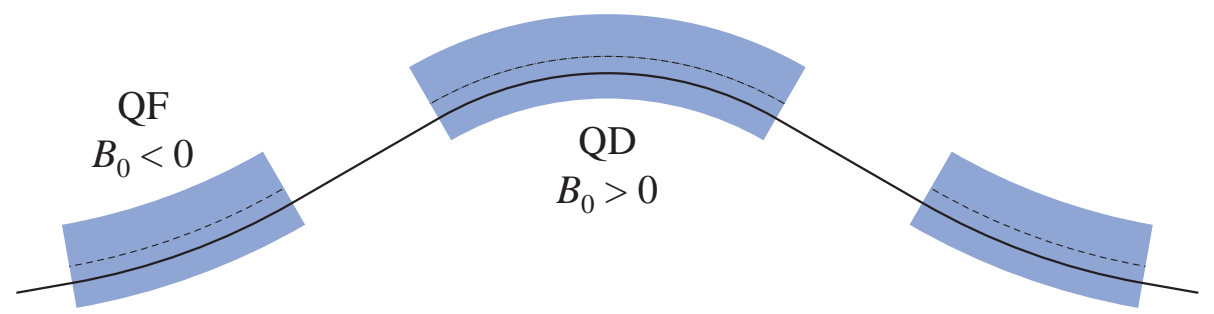

Figure 35: (Color) Geometry of a triplet lattice. The "magnet center offset from reference orbit" listed in Table 12 is positive for all magnets in this diagram. 
Table 12: Parameters for FFAG lattices. (See Fig. 35 to understand the signs of the parameters.)

\begin{tabular}{|c|c|c|c|c|}
\hline Maximum energy gain per cavity (MeV) & \multicolumn{4}{|c|}{7.5} \\
\hline Stored energy per cavity $(\mathrm{J})$ & \multicolumn{4}{|c|}{368} \\
\hline Cells without cavities & \multicolumn{4}{|c|}{8} \\
\hline RF drift length (m) & \multicolumn{4}{|c|}{2} \\
\hline Drift length between quadrupoles (m) & \multicolumn{4}{|c|}{0.5} \\
\hline Initial total energy $(\mathrm{GeV})$ & \multicolumn{2}{|c|}{5} & \multicolumn{2}{|c|}{10} \\
\hline Final total energy $(\mathrm{GeV})$ & \multicolumn{2}{|c|}{10} & \multicolumn{2}{|c|}{20} \\
\hline Number of cells & \multicolumn{2}{|c|}{90} & \multicolumn{2}{|c|}{105} \\
\hline Magnet type & Defocusing & Focusing & Defocusing & Focusing \\
\hline Magnet length (m) & 1.61 & 1.07 & 1.76 & 1.28 \\
\hline Reference orbit radius of curvature $(\mathrm{m})$ & 15.3 & -59.6 & 18.4 & -71.0 \\
\hline Magnet center offset from reference orbit $(\mathrm{mm})$ & -1.6 & 7.7 & 1.1 & 8.7 \\
\hline Magnet aperture radius (cm) & 14.1 & 15.3 & 10.4 & 12.6 \\
\hline Field on reference orbit (T) & 1.6 & -0.4 & 2.7 & -0.7 \\
\hline Field gradient $(\mathrm{T} / \mathrm{m})$ & -9.2 & 8.2 & -15.5 & 12.6 \\
\hline
\end{tabular}

the Study IIa linac. Cavity phases vary linearly along the linac from about $65^{\circ}$ at the beginning to $0^{\circ}$ at the end. As indicated in Table 13, muons are injected into the linac at a momentum of $273 \mathrm{MeV} / \mathrm{c}$, somewhat higher than the average momentum in the cooling channel. To accomplish this, the matching section from the cooling channel to the linac accelerates the beam to the required momentum using rf cavities similar to those in the cooling channel.

The Study IIa RLA following the linac has a low injection energy and accelerates over a small energy range; a dogbone layout is chosen [109]. The dogbone RLA has a single linac that accelerates the beam by just over $1 \mathrm{GeV}$ per pass. For a given amount of installed rf, the dogbone layout has twice the energy separation at the spreaders and recombiners as a racetrack layout, making the switchyard much easier and allowing more passes through the linac. In the design described here, the beam makes 3.5 passes to accelerate from $1.5 \mathrm{GeV}$ to $5 \mathrm{GeV}$. The dogbone arc geometry requires changes in the bend direction twice per arc. Dispersion matching is done by choosing a $90^{\circ}$ phase advance per cell, and removing the dipoles from two consecutive cells. To reduce the effect of a significant phase shift of the reference particle with respect to the cavity phases along the length of the linac on the first pass, the beam is injected halfway down the linac (as shown in Figs. 6 and 34 ).

Tracking of non-scaling FFAG lattices having a linear midplane field profile has shown them to have a good dynamic aperture at fixed energies, provided one is careful to avoid single-cell linear resonances.

Table 13: Linac cryomodule parameters.

\begin{tabular}{lccc}
\hline & Cryo I & Cryo II & Cryo III \\
\hline \hline Length $(\mathrm{m})$ & 3.25 & 4.50 & 6.75 \\
Minimum allowed momentum $(\mathrm{MeV} / \mathrm{c})$ & 273 & 378 & 567 \\
Number of modules & 18 & 12 & 23 \\
Cells per cavity & 1 & 2 & 2 \\
Cavities per module & 1 & 1 & 2 \\
Maximum energy gain per cavity (MeV) & 11.25 & 22.5 & 22.5 \\
RF frequency (MHz) & 201.25 & 201.25 & 201.25 \\
Solenoid length (m) & 1 & 1 & 1 \\
Solenoid field $(\mathrm{T})$ & 2.1 & 2.1 & 2.1 \\
\hline
\end{tabular}


Unfortunately, the tune is not constant in a non-scaling FFAG, so the single-cell tune inevitably passes through many nonlinear resonances. Nonlinearities in the magnetic field due to end effects are capable of driving these nonlinear resonances. ICOOL [105] was used for tracking these non-standard accelerators for several reasons:

- it allows for a fairly arbitrary end-field description;

- it makes that description consistent with Maxwell's equations; and

- it tracks accurately when the lattice acceptances, beam sizes, and energy spread are all large (the situation where most tracking codes fail).

Simple models of both a quadrupole and dipole $\cos \theta$-type magnet, without iron, were used in the tracking studies to date. The models were constructed using the 3D magnet code TOSCA [110]. (Nonlinear fields arising from the end-field fall-off of a dipole or quadrupole are handled properly by ICOOL.) TOSCA is also used to estimate the higher-order multipoles generated by breaking the magnet symmetry at the ends, where the coils form closed loops. For simplicity, the TOSCA computations were done without iron, which results in a predicted overshoot in the field values. To compensate for this, the fields from TOSCA were approximated [22; 23] (see Eq. 30) using functions without the overshoot:

$$
\begin{gathered}
B_{0}(z)=B_{00} \frac{1+\tanh \frac{z}{0.7 R}}{2}, \quad B_{1}(z)=B_{10} \frac{1+\tanh \frac{z}{0.35 R}}{2} \\
B_{2}(z)=-0.2 B_{00} \exp \left[-\frac{1}{2}\left(\frac{z-0.36 R}{0.57 R}\right)^{2}\right],
\end{gathered}
$$

where $R$ is the magnet aperture radius, $B_{0}(z)$ is the dipole field, $B_{00}$ is the dipole field in the center of the magnet, $B_{1}$ is the quadrupole field, $B_{10}$ is the quadrupole field in the center of the magnet, and $B_{2}$ is the maximum magnitude of the sextupole field at the radius $R$.

Initial tracking results indicated that third-order resonances were an issue. As shown in the left hand side of Fig. 36, setting the integrated body sextupole to $68 \%$ of the integrated end sextupoles eliminates the resonance and permits acceleration over the entire 5-10 GeV energy range without losing a high-amplitude particle or having its amplitude grow by a large amount. If the body correction were done imperfectly, there would be significant emittance growth, as seen in the right hand side of Fig. 36.
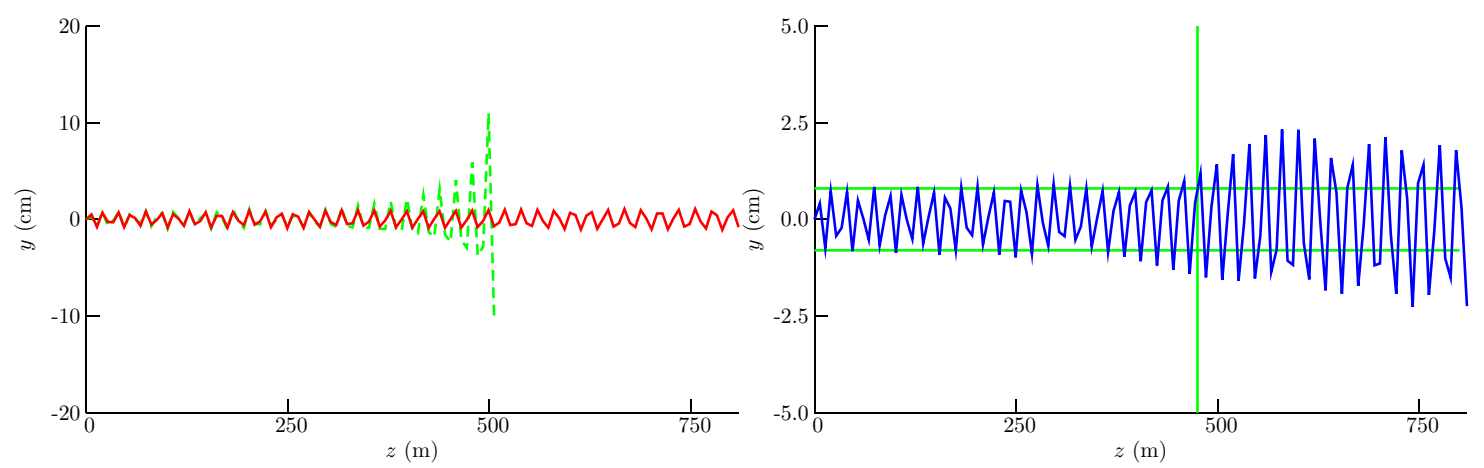

Figure 36: (Color) Tracking of a particle at the edge of the acceptance with uniform acceleration in the 5-10 GeV reference lattice [23]. On the left, the dashed line is without any body sextupole, and the solid line is with the corrected body sextupole. On the right, a smaller integrated sextupole correction (40\% instead of $68 \%$ ) is used, and significant emittance growth is observed. 
Table 14: Parameters of the QD cell [23].

\begin{tabular}{lc}
\hline$E_{\min }(\mathrm{GeV})$ & 10 \\
$E_{\max }(\mathrm{GeV})$ & 20 \\
$L_{0}^{\mathrm{a})}(\mathrm{m})$ & 2 \\
$L_{q}^{\mathrm{b})}(\mathrm{m})$ & 0.5 \\
Type & $\mathrm{QD}$ \\
$L^{\mathrm{c})}(\mathrm{m})$ & 1.762 \\
$r^{\mathrm{d})}(\mathrm{m})$ & 18.4 \\
$X_{0}^{\mathrm{e})}(\mathrm{mm})$ & 1.148 \\
$R^{\mathrm{f})}(\mathrm{cm})$ & 10.38 \\
$\left.B_{0}{ }^{\mathrm{g}}\right)(\mathrm{T})$ & 2.72 \\
$B_{1}^{\mathrm{h})}(\mathrm{T} / \mathrm{m})$ & -15.50 \\
\hline
\end{tabular}

a) $L_{0}$ is the length of the long drift between the QF magnets.

b) $L_{q}$ is the length of the short drift between $\mathrm{QF}$ and QD magnets.

c) $L$ is the length of the reference orbit inside the magnet.

d) $r$ is the radius of curvature of the reference orbit.

e) $X_{0}$ is the displacement of the center of the magnet from the reference orbit.

f) $R$ is the radius of the magnet bore.

g) $B_{0}$ is the vertical magnetic field at the reference orbit.

h) $B_{1}$ is the derivative of the vertical magnetic field at the reference orbit.

\subsubsection{Design of Combined-Function Superconducting Magnet for Non-scaling FFAGs}

An initial design of a superconducting combined-function (dipole-quadrupole) magnet has been developed [111] for one of the QD magnets requiring the highest field and gradient (see Fig. 37). The parameters of the QD cell are listed in Table 14. The magnet design is based on a cosine-theta configuration with two double layers of $\mathrm{Nb}-\mathrm{Ti}$ cable for each function. The quadrupole coil is located inside the dipole coil. Details of the design can be found in Refs. [22; 23]. It is straightforward to adjust the design to cancel the end-field multipoles as proposed in Section 5.2.2.

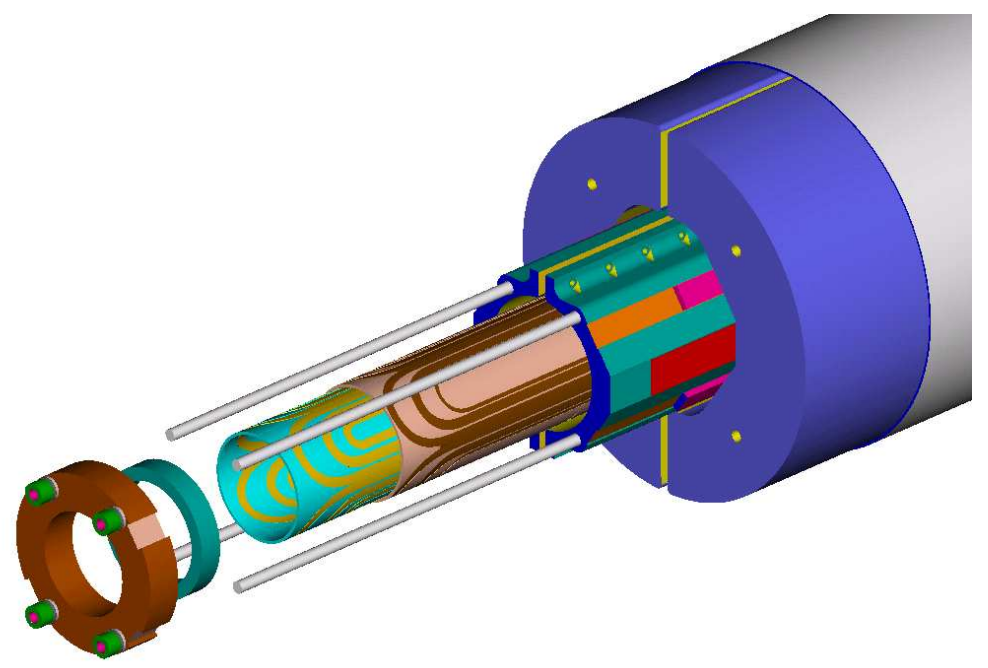

Figure 37: (Color) Exploded view showing the two quadrupole layers (dipole coils not shown). 


\subsection{Muon Storage Ring}

The muon storage ring is the last piece of the NF facility. Rings for $20 \mathrm{GeV}$ and for $50 \mathrm{GeV}$ muons have been designed [21; 30; 112]. Muon decays in the "production" straight section (or sections in the case of a triangular-shaped ring) provide the neutrino flux to the far detector, located several thousand kilometers from the ring. In Study II, the ring was designed for a $20 \mathrm{GeV} \mathrm{NF}$, with a neutrino oscillation baseline of $3000 \mathrm{~km}$. While not a fundamental problem, it is worth noting that, to aim at a detector located some $3000 \mathrm{~km}$ from the ring, it is necessary to slope the production straight section downward by about $13^{\circ}$. For a $7000 \mathrm{~km}$ baseline, the tilt would increase to about $30^{\circ}$. Such a steep slope implies a substantial difference in depth between the two arcs. The installation and alignment of a sloped ring will be somewhat more complicated than for a "flat" ring, and there are potential implications for distributing cryogens around the ring.

An important figure-of-merit for a NF storage ring is the fraction of the circumference that makes up the production region, as this determines the fraction of decays that are useful for the physics program. This fraction is given by:

$$
f_{S}=\frac{L_{S}}{C}=\frac{L_{S}}{2\left(L_{S}+L_{A}\right)},
$$

where $L_{S}$ is the length of one straight section, and $L_{A}$ is the length of $180^{\circ}$ of arc. Plotting this fraction as a function of $L_{S} / L_{A}$, generates the curve shown in Fig. 38. It is clear that the curve is becoming asymptotic beyond $L_{S} / L_{A} \approx 2$, so there is little motivation for the storage ring designers to go much beyond this ratio. To control the overall ring size, it helps to design the ring such that the arcs are reasonably compact.

One way to provide a compact arc design is to adopt high-field magnets. This is the approach followed in Study II [21], where $\mathrm{Nb}_{3} \mathrm{Sn}$ magnets were chosen. This material has the inconvenient physical property of being brittle, which does not lend itself to magnet fabrication. However, with pancake-style coils, it is possible to make high-field magnets with $\mathrm{Nb}_{3} \mathrm{Sn}$ conductor. Even with highfield magnets, there is typically substantial loss of compactness due to the need to provide adequate space between magnets. The space requirement comes partially from the desire to avoid overlapping fields on the magnet coils (which potentially limit the maximum field that can be achieved) and partially from the need for "services" (cold-to-warm transitions and the like) at the end of each magnet cryostat. Minimizing the number of magnet "ends" often motivates the use of combined-function magnets, similar to that described in Section 5.2.3.

A second design issue for a muon storage ring is the need to minimize the beam divergence in the production straight. To yield a minimal flux uncertainty at the detector, the angular spread in the

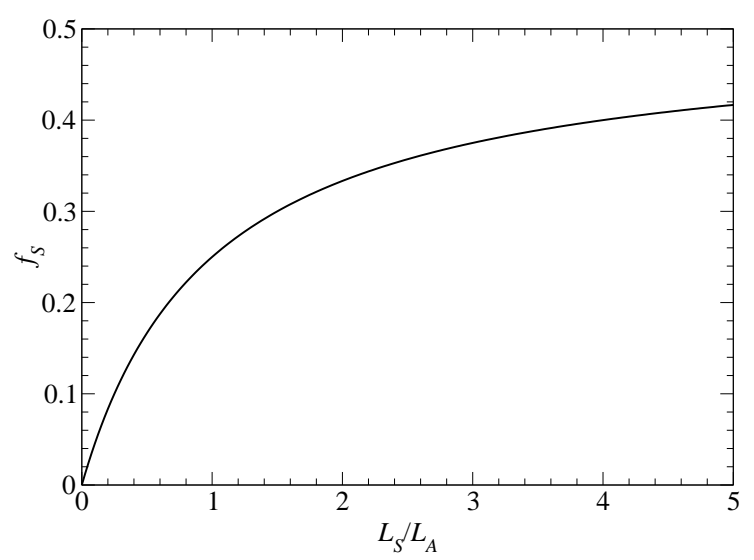

Figure 38: Fraction of decays in a straight $\left(f_{S}\right)$ as a function of the ratio of the length of a straight to the length of an arc. 
beam must be kept well below the angular spread of the neutrinos due to the muon decay kinematics. As a rule of thumb, the ring is generally designed such that $\sigma_{\theta} \leq 0.1 / \gamma$. For the production straight section, this translates into a requirement that the Twiss parameter gamma $\left(\gamma_{T}=\left(1+\alpha^{2}\right) / \beta\right)$ be small. This, in turn, requires that beta be large and alpha small. It should be obvious that these requirements for the production straight section are at odds with what is preferred in the arcs. To keep magnet apertures small, making it more efficient to provide the requisite field, it is best if the arcs have low beta functions. It is possible, though nontrivial, to accommodate both constraints into the optics. However, doing so means that there must be a matching region where the beta functions do not meet the divergence constraint, and it is important for the experiments that this matching region be shielded from a direct "line-of-sight" to the detector. Solutions have been found to handle all of the requirements $21 ;$ 112, although the need to shield the matching region reduces the available length of the production region. The lattice developed for Study II, shown in Fig. 39, has a ratio between the lengths of arcs and straight sections such that the geometric decay ratio, $f_{s}$, is just over 0.35 . The central $93 \mathrm{~m}$ of the production straight has a Twiss gamma function of $0.01 \mathrm{~m}^{-1}$, which meets the requirements for the angular divergence. Note the large difference in beta functions between the downward-going production straight section and the upward-going return straight section. This is typical of a ring where particles of only one sign are intended to circulate, as was the case for the Study II design. If particles of both signs must circulate, then both straight sections are production straights and must have similar optics. To accomplish this, a straightforward redesign of the injection scheme is required.

Chromatic correction of a muon storage ring is not a trivial matter, though the techniques are well understood. The chromatic effects that must be corrected result from both the arc and the straight section quadrupoles. However, the sextupoles needed for chromatic correction must all be located in the arcs, where the dispersion of the ring is non-zero. By design, the arcs are kept small compared with the straight sections, which means that quite strong sextupoles are needed. In general, this has a deleterious effect on dynamic aperture. Another beam dynamics effect comes from the end-sextupoles that arise in a typical magnet design. Using the same approach as in Section 5.2.3, this effect can be compensated. In general, proper chromatic correction depends critically on having the correct phase relationship between sextupoles to cancel geometrical effects. While this is easily achieved for on-momentum particles, the cancellation becomes imperfect for off-momentum particles. Since a muon beam has a relatively large momentum spread, it is a challenge to do a good job of chromatic correction [113]. Fortunately, the demands on dynamic aperture are relatively modest, as particles are only required to survive for roughly 500 turns. Particle tracking must be used to test the adequacy of a particular storage ring design. A realistic evaluation will include all errors, including systematic and random multipole errors in the magnets, random strength errors, and misalignments. As will be discussed in Section 6.2.4, there are tracking codes that work properly in the muon beam parameter regime [114; 115; 116].

Finally, the heat load from muon decay products poses a significant challenge for the magnet de-

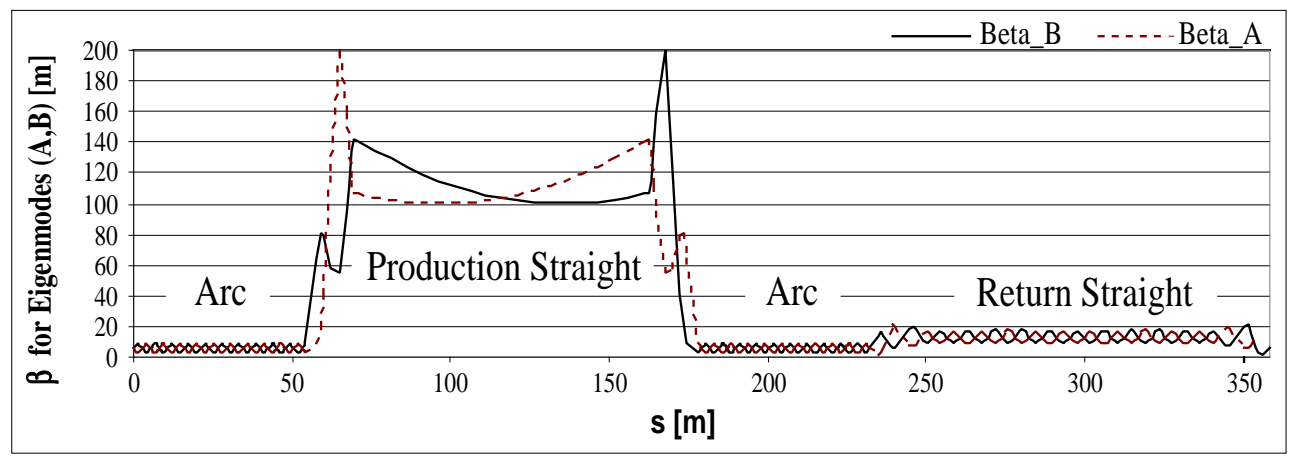

Figure 39: Beta functions for the Study II storage ring. Because the lattice is based on skew quadrupoles, the eigenplanes shown here are rotated $45^{\circ}$ from the normal horizontal and vertical directions. 
signers. It can be in the neighborhood of $40 \mathrm{~W} / \mathrm{m}$ [117], which is too much to comfortably absorb at cryogenic temperatures. As shown in Ref. [117], a modest high- $Z$ liner, e.g., $1 \mathrm{~cm}$ of tungsten, is enough to decrease the heat load by nearly an order of magnitude. The alternative approach, adopted in Ref. [21, is to use pancake dipoles with a gap at the horizontal midplane along with quadrupole magnets with a skew symmetry. In this configuration, there is room to absorb the energy from the decay products at room temperature, which is straightforward.

Although the muon storage ring presents some design challenges, it remains one of the easier, and hence less costly, portions of a NF complex. The main open question is the desired final energy. At present, the candidate energies that have been studied are $20 \mathrm{GeV}$ and $50 \mathrm{GeV}$. It may be that the most effective strategy is to provide a ring that can operate at both these energies in order to optimize the physics program for a given capital expenditure. This question is being considered as part of the International Scoping Study (ISS), discussed in Section 6.2.4.

\section{$6 \quad \mathrm{R} \& \mathrm{D}$}

Successful construction of a NF based on a muon storage ring requires the development and demonstration of many novel techniques, and hence requires an extensive R\&D program. The R\&D issues for each of the major systems must be addressed by a mix of theoretical studies, simulation, component development, and experimental studies, as appropriate. The R\&D is being pursued in Europe, Japan, and the U.S. In the last several years, this $R \& D$ has become increasingly international. Of particular note are three major international R\&D endeavors: $(i)$ a mercury-jet target experiment (MERIT) hosted at CERN 118; 119]; (ii) a muon ionization cooling experiment (MICE) hosted at RAL [120; and (iii) an "International Scoping Study of a Future Neutrino Factory and Superbeam Facility" (ISS) [121] that is preparing the way for a fully international design study in the coming years. These international R\&D efforts are complemented by regional activities, many of which also benefit from significant participation from other regions. The R\&D is presented, and to some extent planned, at the annual NuFact workshops, which rotate annually among Europe, Japan, and the U.S.

In the following, we begin by giving a brief overview of the R\&D program required in the next few years, and then describe, for each R\&D task, recent progress and plans.

\subsection{R\&D Program Overview}

The global R\&D program is designed to answer first the key questions needed to embark upon a "Zeroth-order" Design Report (ZDR), which will examine the complete NF subsystems, making sure that nothing is forgotten, and will show how the parts merge into a coherent whole. The present vision is that this activity will take place in an international context as part of the anticipated "International Design Study" (IDS) that will follow the ISS. After completion of the full R\&D program, it is expected that a formal Conceptual Design Report (CDR) for a NF could be undertaken. The CDR would document a complete and fully engineered design for a facility, permitting a detailed bottom-up cost estimate for all systems.

The overall R\&D program required to prepare for a ZDR, and eventually for a CDR, can be thought of as comprising the following tasks:

\section{Proton Driver Studies}

Development of suitable high-power, multi-GeV proton sources is of interest to several laboratories for reasons that go beyond Neutrino Factories. For example, such development is of interest in the context of upgrades to conventional neutrino beams. Hence, much of the proton driver R\&D is being pursued 
within this broader context. A NF proton source requires a primary beam that is not only high power, but is also delivered in very short bunches. Hence, the NF-specific proton driver studies focus on:

- Production of intense, short proton bunches, e.g., with space-charge compensation, high-gradient, low frequency rf systems and/or adjustments of the momentum compaction factor

\section{Target R\&D for High-Power Beams}

A target must be designed to withstand a proton beam power of up to $\sim 4 \mathrm{MW}$, and its performance must be confirmed experimentally. The ongoing R\&D to achieve this consists of:

- Optimization of target material (low- $Z$ or high- $Z$ ) and form (solid, moving band, liquid-metal jet)

- Design of a high-field solenoid $(\approx 20 \mathrm{~T})$ that must operate in a very high radiation environment

- The MERcury Intense Target (MERIT) experiment, which will study a mercury-jet target exposed to an intense proton beam within a high-field solenoid; this should validate the performance of this type of target

\section{Front-End Design and R\&D}

The design for the NF front end must be cost effective, and the performance of the components required must be demonstrated. For example, in the NF design presented in Section $\underline{5}$, the cooling channel design assumes a normal-conducting rf (ncrf) cavity gradient of $15 \mathrm{MV} / \mathrm{m}$ at $201.25 \mathrm{MHz}$, which is beyond the performance reached to date for cavities in this frequency range. In addition, in the bunching, phase rotation, and cooling channels, the cavities must operate within a multi-Tesla magnetic field. Hence, there is an ongoing and very active hardware development program for the NF front end. Much of the component development and testing is being pursued in the U.S. by MUCOOL [122], a program of the U.S. NFMCC. The main front end R\&D tasks are:

- Design of efficient and cost-effective bunching and phase rotation systems, either based on rf technology or on alternative approaches, e.g., FFAG rings

- Development and testing of high-gradient ncrf cavities, at frequencies in the range of 88-201 MHz, operating in multi-Tesla magnetic fields

- Development and testing of efficient and long-lifetime high-power rf sources at these frequencies

- Development and testing of various absorbers for muon cooling, including $\mathrm{LH}_{2}$ (along with the very thin low- $Z$ containment windows required to minimize scattering within the cooling channel) and $\mathrm{LiH}$

- Development and testing of candidate diagnostics to measure emittance and optimize cooling channel performance

- The Muon Ionization Cooling Experiment (MICE) [120], to validate the implementation and performance of the cooling channel, and to confirm that the simulations of the cooling process are accurate

- Development and testing of high-power rf cavities filled with high-pressure gaseous hydrogen to explore this alternative technology for an ionization cooling channel 


\section{Acceleration Section Design and R\&D}

The NF acceleration scheme must be cost effective, and be able to accelerate a beam with large emittance. The acceleration scenario presented in Section 5 satisfies these requirements, but needs further study. In particular, it demands $10 \mathrm{MV} / \mathrm{m}$ from superconducting rf (scrf) cavities at 201.25 MHz. This gradient has only been reached in one test cavity at low power. The ongoing NF acceleration section R\&D activities consist of:

- Optimization of acceleration techniques to increase the energy of a muon beam (with a large momentum spread) from a few $\mathrm{GeV}$ to a few tens of $\mathrm{GeV}$ (e.g., recirculating linacs [123], very rapidly cycling synchrotrons [124], FFAG rings [31; 125])

- Development of high-gradient scrf cavities at frequencies near $200 \mathrm{MHz}$, along with efficient and long-lifetime high-power sources (about $10 \mathrm{MW}$ peak) to drive them

- Development and testing of new large-acceptance accelerators (scaling and non-scaling FFAGs) and their components

\section{Storage Ring Design}

The storage ring must accept a large-emittance beam, and must maximize the percentage of the ring circumference occupied by the production straight section. The main storage ring R\&D activities are:

- Design and testing of large-aperture, well-shielded superconducting magnets that will operate in the muon-decay radiation environment

- Design and testing of other components (rf cavities, magnets, diagnostics) that will operate in the muon-decay radiation environment

\subsection{R\&D Accomplishments and Plans}

Most of the R\&D activities listed above are already under way, and many are well advanced. Perhaps the most substantial of the ongoing NF-specific R\&D programs are in the areas of targetry, ionization cooling, acceleration, and overall facility design and simulation studies.

\subsubsection{Targetry R\&D}

The BNL Targetry experiment, E951, conducted by the NFMCC, has carried out initial beam tests 126 of both a solid carbon target and a mercury-jet target at a proton beam intensity of up to $4 \times 10^{12} \mathrm{ppp}$. Note that for liquid-metal targets the limiting effects are basically characterized as single-pulse effects, whereas for solid targets, longer-term radiation-damage issues can, over time, lead to the destruction of the target.

In the case of the solid carbon target, the E951 measurements showed that a carbon-carbon composite having nearly zero coefficient of thermal expansion (CTE) is largely immune to beam-induced pressure waves. Target sublimation is still an issue, but a carbon target in a helium atmosphere is expected to have negligible sublimation loss. A program to verify this is under way at ORNL [127; 128]. If radiation damage is the limiting effect for a carbon target, the predicted lifetime would be about 12 weeks when bombarded with a 1 MW proton beam.

In addition to carbon-carbon composite studies, a search is being made for new target materials. Irradiation studies at the level of 0.25 dpa have been carried out for candidate materials by Simos et al. [129] using the BLIP facility at BNL. They have focused on various metals and metal alloys selected for their promise in terms of non-irradiated properties, such as a low CTE or high yield strength. 
It was found that some materials, such as Super-Invar, are very sensitive to radiation effects, while other materials, such as Inconel, are not. In parallel with these material characterization studies after irradiation, a second approach [130] has been to irradiate sample target materials with a very high intensity proton beam, such as that from ISOLDE or that at the Fermilab antiproton source. In this case, one looks for gross failure of the material, e.g., cracking or disintegration, that is, these are essentially destruction tests. Ultimately, the two approaches are complementary - the first one will identify promising candidates and the second one will validate them under more or less "combat" conditions. A new phase of characterization studies, involving more exotic materials (e.g., Ti-6Al-4V, VASCOMAX, AlBeMet, and Toyota "Gum Metal" alloy) is just getting under way.

The most advanced NF target development to date has not been for solid targets, but for mercuryjet targets. The E951 mercury-jet target tests, performed at a beam intensity of about $2 \times 10^{12}$ ppp, showed that the jet is not dispersed until long after the beam pulse has passed through the target. Measurements of the velocity of droplets emanating from the jet after it is hit with an AGS proton beam pulse $(\approx 10 \mathrm{~m} / \mathrm{s}$ for $25 \mathrm{~J} / \mathrm{g}$ energy deposition) compare favorably with simulation estimates. High-speed photographs (see Fig. 40) indicate that the beam disruption at the present intensity does not propagate upstream toward the jet nozzle. If this remains true at the higher intensity of $1.6 \times 10^{13} \mathrm{ppp}$, it will ease mechanical design issues for the nozzle. This will be tested in the MERIT experiment [118; 119], which is scheduled to run at CERN in 2007. Although the mercury jet in the E951 experiment was not injected into a magnetic field, a CERN-Grenoble group has studied the injection of a free mercury jet into a high-field solenoid, and observed that the field appears to improve the quality of the jet by damping surface waves [131].

The MERIT experiment will test a mercury-jet target injected into a $15 \mathrm{~T}$ magnetic field and exposed to a beam with a single-pulse intensity corresponding to bombardment by a $4 \mathrm{MW}$ proton beam. A suitable cost-effective 15-T magnet has been fabricated and is now in the final stages of testing (see left side of Fig. 41). A closed Hg-jet system, capable of providing a jet velocity of $20 \mathrm{~m} / \mathrm{s}$, is being developed for the experiment, as shown in the right side of Fig. 41 and illustrated in Fig. 42. To complement the experimental program, target simulation efforts are ongoing. These aim at a sufficiently detailed understanding of the processes involved to reproduce the observed experimental results both with and without a magnetic field. Fully 3D magneto-hydrodynamics codes [132] are being utilized for this effort.

At present, there is no program to test a NF solid target in a realistic configuration, that is, to carry out the solid-target experiment equivalent to MERIT. This will likely be needed, however, if it is desired to demonstrate that a solid target is practical at the power levels of $\sim 4 \mathrm{MW}$ suitable for a NF.

Finally, an alternative mercury-jet concept, being explored initially for the PRISM project, is to use a conducting $\mathrm{Hg}$ target [133] that is pulsed with a very high (on the order of $1 \mathrm{MA}$ ) current. The idea is that the secondaries will be focused by the toroidal magnetic field produced by the current pulse, resulting in a relatively low-emittance beam. The material properties of the target will be greatly stressed, both by the beam and by the pulsed current, so it remains to be seen whether this interesting

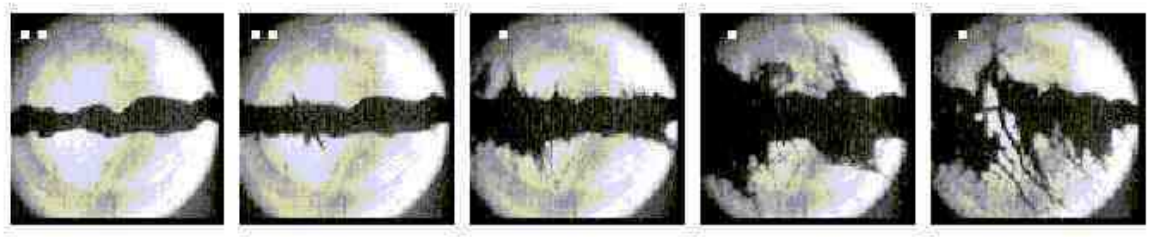

Figure 40: Disruption of Hg jet hit with an AGS beam bunch containing $2 \times 10^{12}$ protons. Frames from left to right correspond to time steps of $0,0.75,2,7$, and $18 \mathrm{~ms}$, respectively [22]. 

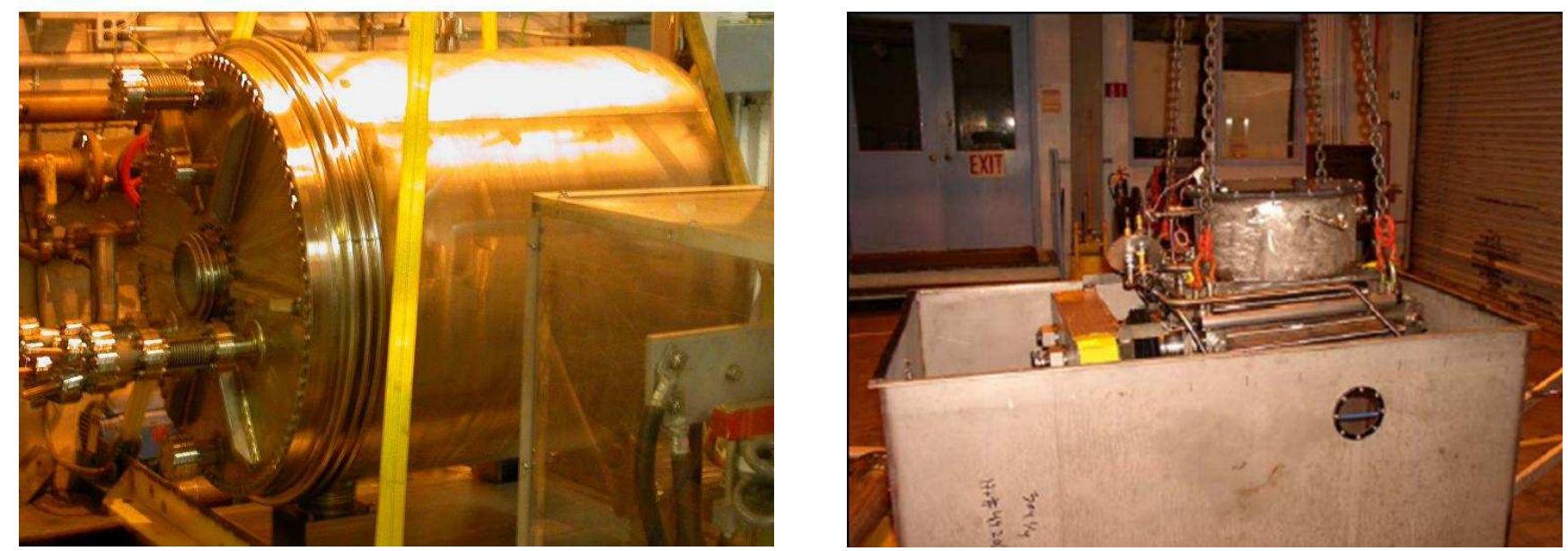

Figure 41: (Color) (Left) 15-T MERIT solenoid under test at MIT. Three nested coils permit operation at 5, 10, and $15 \mathrm{~T}$. The coils are normal conducting but cooled to liquid-nitrogen temperature to ease the requirements on the power supply. (Right) Hg-jet syringe pump system being placed in containment vessel at ORNL.

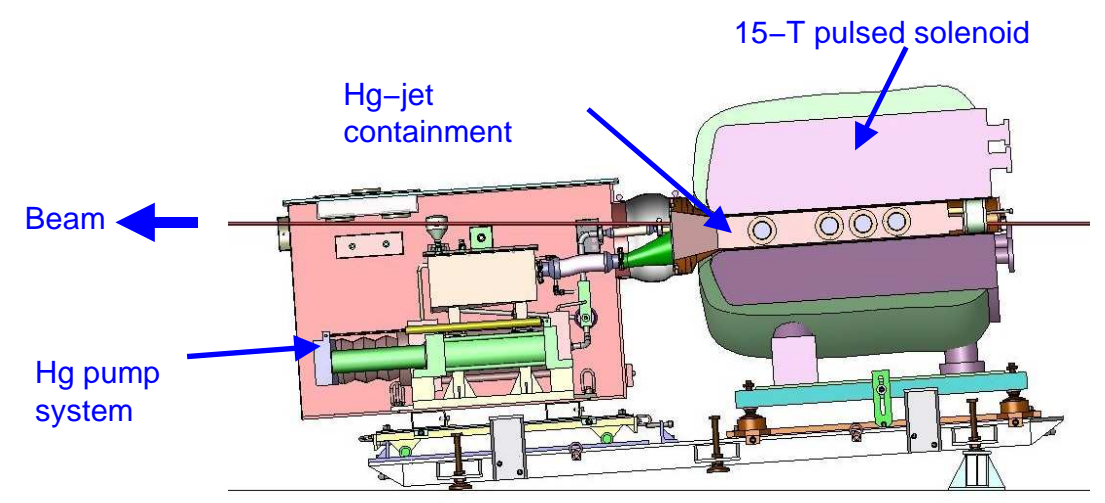

Figure 42: (Color) Illustration of MERIT setup in the TT2A line at CERN.

concept is practical.

\subsubsection{Ionization Cooling Channel R\&D}

Perhaps one of the most challenging of the requirements that must be satisfied for the successful operation of an ionization cooling channel is the need to operate high-gradient rf cavities in a multi-Tesla magnetic field. The first phase of an effort to carry out high-power tests of $805-\mathrm{MHz}$ rf cavities was recently completed in the Lab G test area at Fermilab. A 5-T test solenoid for the facility, capable of operating either in solenoid mode (its two independent coils powered in the same polarity) or gradient mode (with the two coils opposed), was used to study the effects of magnetic field on cavity performance. Both an open-iris multicell $805-\mathrm{MHz}$ cavity, and a single-cell $805-\mathrm{MHz}$ pillbox cavity having Be foils to close the beam irises, have been constructed and tested. The latter cavity permitted an assessment of the behavior of the foils under rf heating and was used to study dark current effects [134]. The cavity reached $40 \mathrm{MV} / \mathrm{m}$ (exceeding its design specification) in the absence of a magnetic field, but was limited by breakdown to less than $15 \mathrm{MV} / \mathrm{m}$ at high magnetic field $(\approx 2.5 \mathrm{~T})$.

Understanding the effects of the magnetic field on cavity performance is crucial. For these studies, a new area, the MUCOOL Test Area (MTA), is now operational at FNAL. The MTA is located at 
the end of the Fermilab $\mathrm{H}^{-}$linac, and is designed to eventually permit beam tests of components with $400 \mathrm{MeV}$ protons. The MTA provides access to both $805-\mathrm{MHz}$ and 201-MHz high-power rf amplifiers for continuing rf tests of the $805-\mathrm{MHz}$ pillbox cavity and for testing a newly constructed $201-\mathrm{MHz}$ cavity (see Fig. 43). The 201-MHz cavity, including its 420-mm diameter curved beryllium windows, was fabricated by a group from LBNL, Jlab, and the University of Mississippi [135]. This cavity, which serves as a prototype for the MICE cavities, underwent successful initial testing during 2006. The tests provide information on the background levels that will be experienced by the MICE detectors when the cavities are operated at high gradient. To study its performance in the magnetic-field configuration to be used in MICE, the 201-MHz cavity must ultimately be tested within a large-bore superconducting solenoid ("coupling coil") that provides the appropriate magnetic field.

The development of high-gradient cavities that operate within a suitable magnetic field, and that meet the NF requirements, will likely be successful. Nonetheless, an alternative technology is being explored by an R\&D company, Muons, Inc. This company is studying cavities filled with high-pressure gaseous hydrogen [136. The concept is to use the high-pressure hydrogen to suppress rf breakdown via the Paschen effect while also using it as a fully distributed absorber (in effect, carrying the approach chosen in Study IIa to its logical conclusion). If the magnetic fields can be configured properly, using this technology to develop a compact method for 6D cooling of muons (that is, longitudinal as well as transverse cooling) may well be possible - a very attractive option. Initial tests are being carried out at Fermilab with an $805-\mathrm{MHz}$ high-pressure cavity, with encouraging results. A gradient of $\approx 60 \mathrm{MV} / \mathrm{m}$ was obtained [136] for molybdenum electrodes after only brief rf conditioning.

In addition to rf cavity R\&D, the MUCOOL program has also pursued the development of a prototype $\mathrm{LH}_{2}$ absorber, the material chosen for Study II and also for MICE [120]. A prototype absorber, constructed at KEK, has been successfully filled with $\mathrm{LH}_{2}$ in the MTA. Several large diameter, thin (125-350 $\mu \mathrm{m})$ aluminum windows have been fabricated by machining from solid disks. These have been pressure tested with water and found to break at a pressure consistent with finite-element design

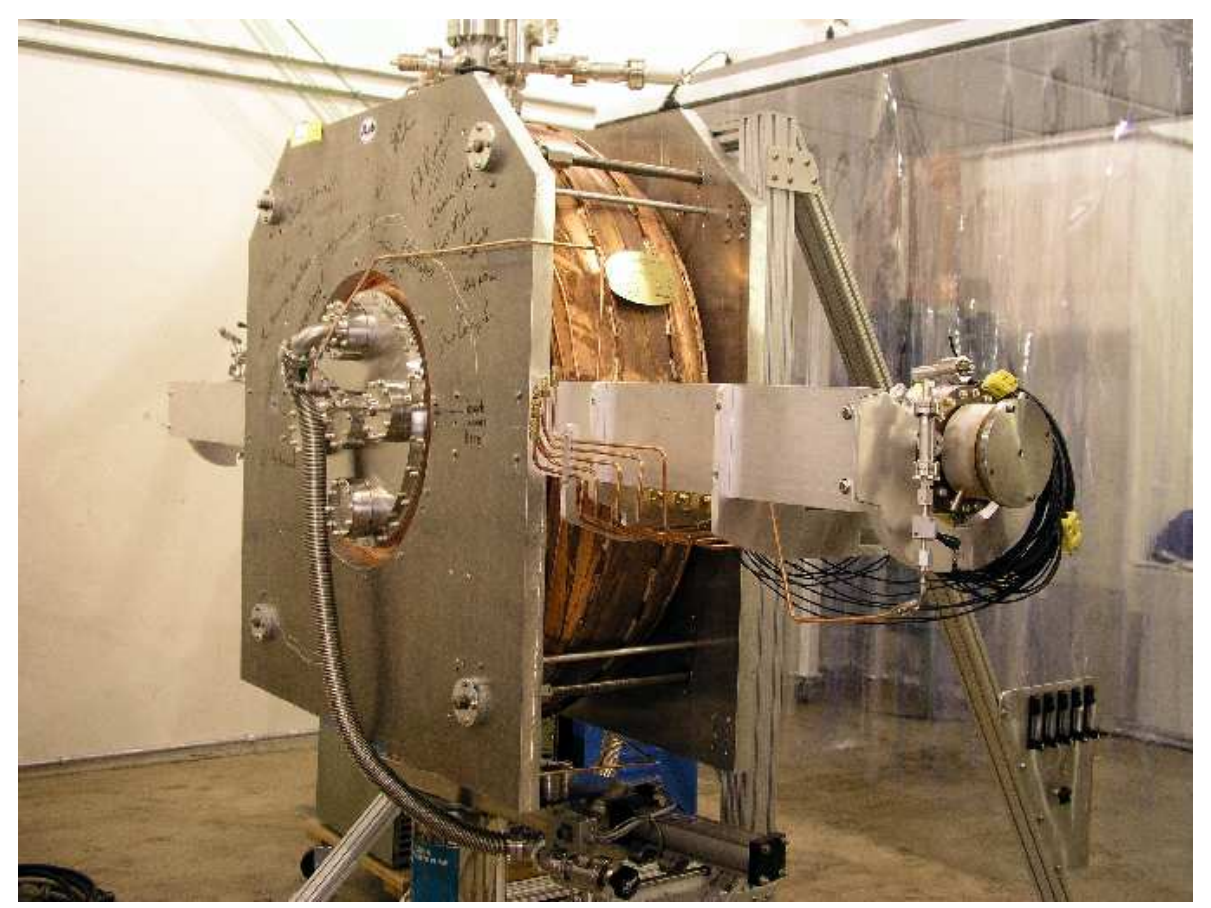

Figure 43: (Color) $201 \mathrm{MHz}$ rf cavity. The 1.4-m-diameter cavity has curved Be windows and is provided with a thin exit port to permit dark current studies. 
calculations [137]. Absorbers that will use these thin windows are also being tested at the MTA. In addition to $\mathrm{LH}_{2}$, another absorber material that must be studied is $\mathrm{LiH}$, the material on which the cooling channel described in Section 5 is based. In the proposed scheme, the LiH (sandwiched between beryllium foils) serves both as an absorber and an rf window. This configuration must be tested in the $805-\mathrm{MHz}$ pillbox cavity described above. $\mathrm{LiH}$ absorber development is expected to begin soon.

The next big step in the global cooling channel R\&D program will be the MICE experiment. MICE was launched at the NuFact01 Workshop in Japan, where a volunteer organization was created to organize a cooling demonstration experiment. Membership in this group included representatives from Europe, Japan, and the U.S. There is now a formal international collaboration, numbering some 140 members from the three geographical regions. The MICE Collaboration [120; 138] has received scientific approval for the experiment from RAL management, and is now funded for "Phase 1" and in the process of seeking the remainder of the funds needed to complete "Phase 2" of the experiment. MICE will involve measuring, on a particle-by-particle basis, the emittance reduction produced by a single cell of the Study II cooling channel. A drawing of the layout is shown in Fig. 44. The cooling channel cell is preceded and followed by identical detector modules that accomplish particle identification and emittance measurement. Provision for testing a series of absorber materials, including both $\mathrm{LH}_{2}$ and solid absorbers, has been made. A safety review of the liquid-hydrogen system has been successfully passed, and detailed engineering has been completed. A first-article liquid-hydrogen handling system will be constructed during 2007 to measure its operating characteristics and parameters. A muon beam line at RAL is under construction, and is expected to deliver first beam to the experiment in August 2007. Fabrication of both the scintillating-fiber tracker and the spectrometer solenoid in which it is mounted are under way.

We can anticipate that the MICE program will be completed by about 2010, or shortly thereafter. At that time the MICE setup (beam and instrumentation) would in principle be available for further component testing and optimization in the context of a preconstruction R\&D phase.

\subsubsection{Acceleration System R\&D}

Ongoing R\&D for muon acceleration at a NF includes both component development and the development of new acceleration schemes based on FFAGs. The component R\&D carried out at Cornell [139] has focused on the development of a high-gradient 201-MHz scrf cavity. A test area of suitable dimensions was constructed and used to test a prototype cavity fabricated for the Cornell group by CERN

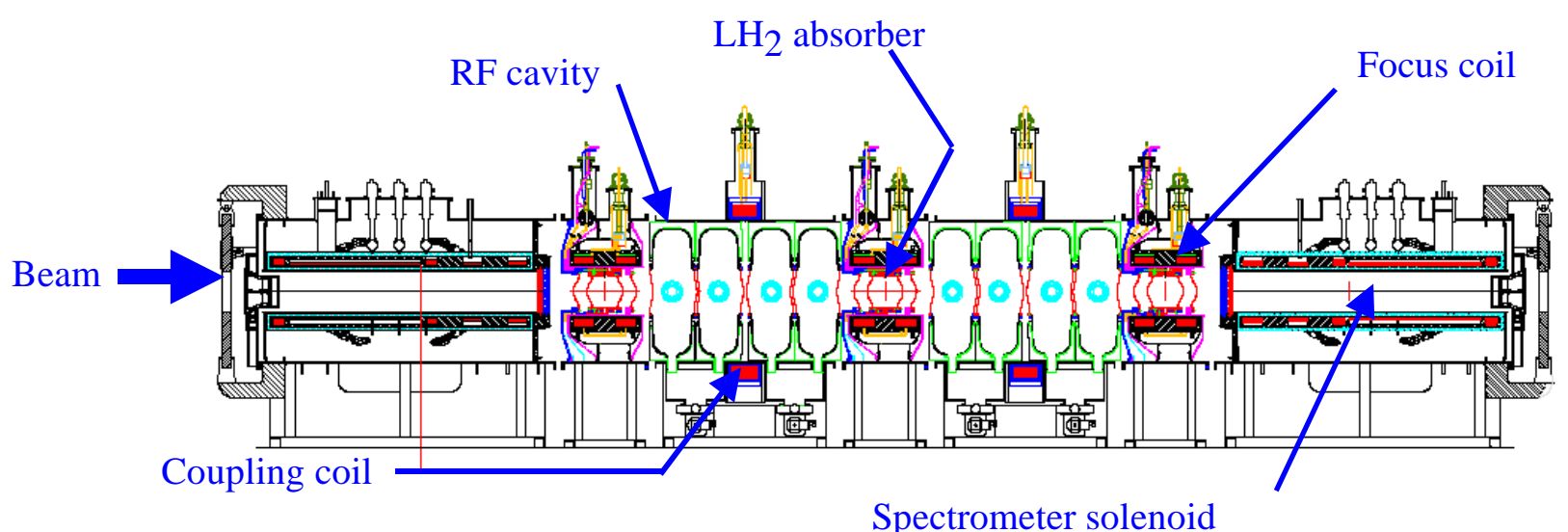

Figure 44: (Color) Diagram of the MICE layout. 
colleagues. The cavity reached $11 \mathrm{MV} / \mathrm{m}$ in initial tests, but exhibited a significant " $Q$ slope" as the gradient increased [108]. To better understand the origins of this phenomenon, effort is shifting to studies on a smaller 500-MHz cavity. Different coating, cleaning, and manufacturing techniques will be explored to learn how to mitigate the observed $Q$ slope. Other fabrication materials, such as explosionbonded $\mathrm{Nb}$ on copper, will be studied to see if this material behaves more like the bulk Nb used very successfully in high-frequency scrf cavities. Detuning issues at $201 \mathrm{MHz}$ associated with the very large cavity dimensions and the pulsed rf system will be evaluated. To demonstrate the ability to adequately reduce nearby magnetic fields in a realistic lattice configuration, tests of the $201-\mathrm{MHz}$ scrf cavity will include operation in the vicinity of a shielded solenoid magnet.

If funding permits, acceleration-system component R\&D activities will be extended to include design of a prototype high-power rf source, which would be explored in collaboration with industry. This source - presently envisioned to be a multibeam or a hollow-beam klystron-must be developed for operation at two different duty factors, because the cooling channel requires a duty factor of about 0.002 whereas the acceleration chain requires 0.045 .

The FFAG R\&D to date has been mostly conducted in Japan, where construction of the PRISM [26] FFAG system is under way. This will be the first test of a so-called scaling FFAG for muons, though several proton systems have already been constructed and successfully commissioned [125]. The PRISM project requires building not only the FFAG ring itself but its injection and extraction systems. Completion of this experiment in a few years will provide important information about both the technical performance and the construction costs of scaling FFAG systems. Moreover, development [140] of a magnetic alloy cavity for PRISM is a step toward demonstrating the concepts needed for effective use of the scaling FFAG rings proposed for a NF by the NuFact-J team [31]. In addition to the scaling FFAG schemes developed in Japan, a new non-scaling FFAG concept has emerged from NFMCC and European studies. An experimental effort, EMMA [141; 142], aimed at building an electron model of a non-scaling FFAG, has been proposed, but is not yet approved. EMMA would be another example of a fully international R\&D effort.

\subsubsection{Design, Simulation, and Theoretical Studies}

Subsequent to work on Study II, the recent effort has focused on further optimization of NF performance and costs. The more cost-effective front-end design described in Section 5 is a result of this work. As an international follow-on to this activity, the ISS [121] was launched and recently completed. This year-long study reviewed the various approaches to building a NF and selected an optimal facility configuration for further detailed study. The ISS issued its preliminary report in talks at the NuFact06 workshop in August 2006. Their findings will be briefly summarized in Section 6.2 .5 below. It should be noted that much of the progress between the Study II and IIa designs is associated with the development of a more effective larger-acceptance acceleration scheme. However, this scheme required further study, which was carried out within the context of the ISS. Further effort on beam dynamics studies in the FFAG rings, including realistic magnetic-field and alignment errors, is urgently needed. For example, assessment of field-error effects on the beam transport must be made to define acceptance criteria for the magnets. This will require use of sophisticated tracking codes, such as COSY [114], Zgoubi [115], or PTC [116], that permit rigorous treatment of field errors and fringe-field effects.

In many ways, the storage ring is one of the most straightforward portions of a NF complex. However, beam dynamics is an issue as the muon beam must circulate for many hundreds of turns. Here too, use of a sophisticated 6D tracking code, is required to assess fringe-field and large-emittance effects. As with the FFAG rings, the relatively large emittance and large energy spread enhance the sensitivity to magnetic-field and magnet-placement errors. Suitable magnet designs are needed, with the main technical issue being the relatively high radiation environment. Another lattice issue that must be studied is polarization measurement. In the initial implementation of a NF it is expected that polarization will 
not be considered, but its residual value may nonetheless be important in analyzing the experiment.

The ISS will lead, beginning in 2007, to a so-called "International Design Study" (IDS) of an optimized NF. This study, in a broad sense an international version of the two previous U.S. feasibility studies, will be organized jointly by representatives from Europe, Japan, and the U.S.

\subsubsection{Initial Findings of the ISS}

The ISS examined the various existing NF designs and compared them to determine which were the most promising. A few key findings [143] are summarized briefly below:

- The optimum energy for the proton driver is $10 \pm 5 \mathrm{GeV}$. Lower energies show a marked decrease in production; higher energies show a modest decrease in production and are not beneficial unless the proton driver has other applications that need the increased energy.

- The Hg-jet target gives optimal muon production for protons in the preferred energy range.

- The Study IIa front end design (described in this paper) is the preferred configuration. Operating simultaneously with both signs of muon beam is favored.

- The non-scaling FFAG beam dynamics issues, in particular the dependence of the longitudinal dynamics on transverse amplitude, limit performance. Cascading more than two such FFAGs looks problematical. A more robust acceleration scheme has been proposed that makes use of two cascaded dogbone RLAs, followed by a single FFAG to reach $25 \mathrm{GeV}$. An additional FFAG stage may be possible, but further work is needed to verify that. The possibility of using a scaling FFAG stage with high-frequency rf and a "harmonic number jump" acceleration scheme is also under study.

- Both racetrack and triangle storage rings are being investigated. The triangle shape is more efficient if there are two suitable baselines that can operate simultaneously. A pair of triangle rings is needed to accommodate simultaneous operation with both signs of muon. A pair of racetrack rings is needed to service two detector baselines. This configuration has no directional constraints on where the detectors are located. Each racetrack ring can accommodate both signs of muon beam aimed at a single detector.

We conclude from the above list that the representative configuration described in this article is in rather good agreement with the optimization found during the ISS. The main differences are that the proton driver energy in Study IIa is somewhat too high and the acceleration scheme, based on two cascaded non-scaling FFAGs, is less robust than seems desirable given our present understanding of FFAG beam dynamics. It is anticipated that the IDS will make these changes in the next phase of NF optimization.

\section{Summary and Future Plans}

Neutrino Factories offer an exciting option for the long-term neutrino physics program. A muon storagering based NF requires $(i)$ the production and collection of a large number of low energy charged pions, (ii) a pion decay channel designed to capture the daughter muons, (iii) a phase rotation and bunching system designed to reduce the muon energy spread and confine the muons within bunches, $(i v)$ an acceleration scheme with a large acceptance to enable muons occupying a relatively large transverse phase space to be accelerated to high energy, and $(v)$ a storage ring with long straight sections within which the decaying muons produce a beam of neutrinos and anti-neutrinos. In addition, in the U.S. 
and CERN NF designs, just before the acceleration systems there is an ionization cooling channel that reduces the transverse phase space occupied by the muons and increases the number of particles accepted by the accelerators.

The performance of various NF designs based on these ingredients has been simulated. Depending on the details, we can expect between $10^{20}$ and $10^{21}$ muon decays per year to contribute to the neutrino beam downstream of each long straight section. The NF physics studies conducted so far suggest that the desired energy for the stored muons is somewhere between $20 \mathrm{GeV}$ and $50 \mathrm{GeV}$. The resulting neutrino beam will have a well understood flux and spectrum, with low systematic uncertainties. Furthermore, in contrast to conventional neutrino beams, NF beams contain $\nu_{e}\left(\bar{\nu}_{e}\right)$ as well as $\bar{\nu}_{\mu}\left(\nu_{\mu}\right)$. This will enable the search for, and measurement of, $\nu_{e} \rightarrow \nu_{\mu}$ and $\bar{\nu}_{e} \rightarrow \bar{\nu}_{\mu}$ transitions with extremely small backgrounds and very high precision. It will also enable the search for, and measurement of, $\nu_{e} \rightarrow \nu_{\tau}$ and $\bar{\nu}_{e} \rightarrow \bar{\nu}_{\tau}$ transitions. The presence of $\nu_{\mu}\left(\bar{\nu}_{\mu}\right)$ in the initial beam will enable $\nu_{\mu}\left(\bar{\nu}_{\mu}\right)$ disappearance measurements to be made, together with the precise measurement of $\nu_{\mu} \rightarrow \nu_{\tau}\left(\bar{\nu}_{\mu} \rightarrow \bar{\nu}_{\tau}\right)$ transitions. Thus, there is a wealth of very precise neutrino oscillation measurements that can be made at a NF. Various physics studies have shown that, for values of $\theta_{13}$ as low as $\mathcal{O}\left(10^{-4}\right)$ or possibly a little lower, the unknown mixing angle $\theta_{13}$ can be measured, the mass hierarchy determined, and a sensitive search for $C P$ violation made. Hence, a NF is considered to be the ultimate tool for studying neutrino oscillations.

Neutrino Factory design studies conducted over the last few years have been sufficiently detailed to define the hardware R\&D needed before this new type of neutrino facility can be constructed. This has led to two very substantial R\&D activities, and for each of these a significant international accelerator R\&D experiment. The first activity is focused on the development of a suitable target-system for the primary proton beam. This has resulted in the development and testing of a liquid-Hg jet injected into a high-field solenoid, and exposed to a high-intensity proton beam. The MERIT experiment, which has been approved at CERN and is expected to run in 2007, is the final step foreseen in this R\&D program. The MERIT experiment will test the response of a $20 \mathrm{~m} / \mathrm{s}$ liquid-Hg jet injected into a 15-T solenoid and exposed to an intense proton beam from the CERN PS. The second major R\&D activity is focused on the development of an ionization cooling channel for a NF. The MUCOOL program in the U.S. is developing and testing the required absorbers and rf cavities, which must operate in close proximity within a high-field solenoid. Perhaps the most challenging hardware issue identified to date is related to the operation of high-gradient cavities in high magnetic fields. The remainder of the MUCOOL R\&D program is crucial to obtaining an adequate understanding of this. The first phase of the MICE experiment, which has been approved at RAL and is expected to begin operation in 2007, will prepare the way to test a short cooling channel section in a muon beam. It is hoped that this test, the second phase of MICE, will be conducted by about the end of 2010 .

In addition to the globalization of the NF hardware R\&D program, a significant step has recently been made towards a global NF design study. The one-year-long International Scoping Study is now completed. In addition to beginning the process of comparing the various competing NF designs, the ISS has also built an international team that will form the basis for a subsequent several-year-long International Design Study that will be focused on making the optimal technology choices.

The recent progress towards a realistic and cost-effective NF has been encouraging. The globalization of the R\&D program, the approval of the proposals for its two significant international accelerator R\&D experiments, and the completion of the International Scoping Study, are significant achievements. We anticipate that by 2010-2012 this phase of the R\&D will be complete. At that time we would hope that there will be a cost-effective NF design based upon proven technology, a solid basis for making a reliable cost estimate, and a good understanding of NF performance. The NF will then be ready for a community decision on whether this new facility should be inserted into the high-energy physics roadmap, and hence whether to proceed with the final pre-construction $R \& D$.

Complementing the accelerator-related R\&D program, studies of detector design and performance have also gotten under way as part of the ISS. This important effort will continue - and hopefully 
expand-during the upcoming IDS activity.

\section{Acknowledgments}

This work was supported by the Fermi National Accelerator Laboratory, which is operated by Universities Research Association, under contract No. DE-AC02-76CH03000 with the U.S. Department of Energy, and also by Lawrence Berkeley National Laboratory, which is operated by the University of California, under contract No. DE-AC02-05CH11231 with the U.S. Department of Energy. We are very grateful to Ina Reichel for her considerable help with preparation of the document. We also thank the many colleagues worldwide who have contributed to the progress summarized here, especially those who participated in Study II and its update, Study IIa.

\section{References}

[1] M. M. Alsharo'a et al., Recent Progress in Neutrino Factory and Muon Collider Research within the Muon Collaboration. Phys. Rev. ST Accel. Beams, 6:081001, 2003.

[2] S. Geer, The Physics Potential of Neutrino Beams from Muon Storage Rings, 1997, FERMILABPUB-97-389.

[3] S. Geer. Phys. Rev., D57:6989, 1998, ibid. 59, 039903E (1999).

[4] R.M. Dzhilkibaev and V.M. Lobashev. Sov. J. Nucl. Phys., 49(2):384, 1989.

[5] R. Palmer et al., 1995, BNL-61581.

[6] A. A. Kolomensky, On the Oscillation Decrements in Accelerators in the Presence of Arbitrary Energy Losses. Sov. Atom. Energ., 19:1511-1513, 1965.

[7] A. Skrinsky and V. Parkhomchuk. Sov. J. Part. Nucl., 12:223-247, 1981.

[8] D. Neuffer, Principles and Application of Muon Cooling. In Proceedings of the 12th Int. Conf. High Energy Accelerators, (p. 481).

[9] D. Neuffer. Part. Accel., 14:75, 1983.

[10] D. Cline, ed., 2nd Workshop on the Physics Potential and Development of $\mu^{+} \mu^{-}$Colliders, 1995, AIP Conference Proceedings.

[11] R. Palmer et al., $\mu^{+} \mu^{-}$Colliders: A Feasibility Study, 1996, BNL-52503, FERMILAB-CONF96/092, LBNL-38946.

[12] S. Geer and R. Raja, eds., Workshop on Physics at the First Muon Collider and at the Front End of a Muon Collider, 1997, AIP Conference Proceedings 435.

[13] A. D. Rujula, M. B. Gavela, and P. Hernandez, Neutrino Oscillation Physics with a Neutrino Factory. Nucl. Phys., B547:21, 1999, hep-ph/9811390.

[14] V. Barger, S. Geer, and K. Whisnant, Long Baseline Neutrino Physics With a Muon Storage Ring Neutrino Source. Phys. Rev. D, 61:053004, 2000.

[15] L. Wolfenstein. Phys. Rev. D, 17:2369, 1978.

[16] S. P. Mikheyev and A. Smirnov. Yad. Phys., 42:1441, 1985. 
[17] Y. Fukuda et al., Evidence for Oscillation of Atmospheric Neutrinos. Phys. Rev. Lett., 81:15621567, 1998, hep-ex/9807003.

[18] The Neutrino Factory and Muon Collider Collaboration page, http://www.cap.bnl.gov/mumu/.

[19] N. Holtkamp and D. Finley, eds., A Feasibility Study of a Neutrino Source Based on a Muon Storage Ring. Fermilab-Pub-00/108-E, Fermilab, 2000, http://www.fnal.gov/projects/muoncollider/nu-factory/nu-factory.html.

[20] S. Geer, H. Schellman, eds., Physics at a Neutrino Factory. Technical report, Fermilab, 2000, FERMILAB-FN-692, hep-ex/0008064.

[21] S. Ozaki, R. Palmer, M. Zisman, and J. Gallardo, eds., Feasibility Study-II of a Muon-Based Neutrino Source. BNL-52623, 2001, http://www.cap.bnl.gov/mumu/studyii/FS2-report.html.

[22] S. Geer and M. Zisman, eds., Neutrino Factory and Beta Beam Experiments and Development. BNL-72369-2004, FERMILAB-TM-2259, LBNL-55478, note = http://www .aps .org/neutrino/ loader.cfm?url=/commonspot/security/getfile.cfm\&PageID=58766, 2004.

[23] J.S. Berg, S.A. Bogacz, S. Caspi, J. Cobb, R.C. Fernow, J.C. Gallardo, S. Kahn, H. Kirk, D. Neuffer, R. Palmer, K. Paul, H. Witte, and M.S. Zisman. Phys. Rev. Special Topics-AB, 9:011001, 2006.

[24] http://muonstoragerings.web.cern.ch/muonstoragerings/.

[25] http://hepunx.rl.ac.uk/neutrino-factory/.

[26] http://www-prism.kek.jp/nufactj/index.html.

[27] B. Autin, A. Blondel, and J. Ellis, eds., Prospective Study of Muon Storage Rings at CERN. CERN 99-02, 1999.

[28] Y. Mori. J. Phys. G, 29:1527, 2003.

[29] HEPAP White Paper: Planning for U.S. High-Energy Physics. http://www.science.doe.gov/ hep/hepap_reports.shtm, 2000, DOE/SC-0027.

[30] P. Gruber et al., The Study of a European Neutrino Factory Complex. http://slap.web.cern. ch/slap/NuFact/NuFact/nf122.pdf, 2002.

[31] NuFactJ Working Group, A Feasibility Study of a Neutrino Factory in Japan. http://www-prism. kek.jp/nufactj/nufactj.pdf, May 242001.

[32] The Proton Driver Design Study. FERMILAB-TM-2136, 2000.

[33] Proton Driver Study II. FERMILAB-TM-2169 (Parts I and II), 2002.

[34] N. Mokhov, Particle Production and Radiation Environment at a Neutrino Factory Target Station. In Proceedings of the 2001 Particle Accelerator Conference, (p. 745), 2001, see also http:// www-ap.fnal.gov/MARS/.

[35] J. Miller et al. IEEE Trans. Magn., 30:1563, 1994.

[36] N. Mokhov. http://www-ap.fnal.gov/MARS/, nucl-th/9812038. 
[37] Y. Yamazaki et al., Accelerator Technical Design Report for J-PARC. Technical report, KEKREPORT-2002-13, 2002.

[38] T. Gaisser, Cosmic Rays and Particle Physics. Cambridge University Press, 1990.

[39] A. Blondel, Muon Polarization in the Neutrino Factory. Nucl. Instrum. \& Meth., A451:131-137, 2000, proceedings of the ICFA/ECFA Workshop NuFact'99: Neutrino Factories based on Muon Storage Rings, Lyon, France.

[40] R. Fernow, J. Gallardo, and Y. Fukui, Polarization Effects in the Front End of the Neutrino Factory. Nucl. Instrum. \& Meth., A472:541-545, 2001, proceedings of the International Workshop NuFact'00: Muon Storage Ring for a Neutrino Factory, Monterey, CA.

[41] D. MacFarlane et al. Z. Phys., C26:1, 1984, J.P. Berge et al., Z. Phys. C35, 443 (1987), J.V. Allaby et al., Z. Phys. C38, 403 (1988), P. Auchincloss et al., Z. Phys. C48, 411 (1990), world average from J. Conrad, M. Shaevitz and T. Bolton, Rev. Mod. Phys. 70, 1341 (1998).

[42] MINOS Technical Design Report. http://www.hep.anl.gov/ndk/hypertext/minos_tdr.html, nuMI-L-337 TDR.

[43] V. Barger, S. Geer and K. Whisnant. Phys. Rev., D61:053004, 2000, hep-ph/9906487.

[44] C. Crisan and S. Geer. Technical report, FERMILAB-TM-2101, 2000.

[45] I. Papadopoulos, Impact of the Muon Beam Divergence and Polarization on the Prediction Accuracy of the Neutrino Flux at a Distant Detector of a Neutrino Factory. Nucl. Instrum. E Meth., A472:455-459, 2001, proceedings of the International Workshop NuFact'00: Muon Storage Ring for a Neutrino Factory, Monterey, CA.

[46] P. Minkowski. Phys. Lett., B67:421, 1977.

[47] P. Ramond, The family group in grand unified theories. In Invited talk given at Sanibel Symposium, Palm Coast, Fla., February 25 - March 2, 1979.

[48] M. Gell-Mann, P. Ramond, and R. Slansky, Complex Spinors and Unified Theories. Supergravity. North Holland, Amsterdam, 1979.

[49] T. Yanagida, 1979, Proceedings of the Workshop on Unified Theory and Baryon Number in the Universe, KEK.

[50] S. L. Glashow, The future of elementary particle physics. In M. Lévy, J.-L. Basdevant, D. Speiser, J. Weyers, R. Gastmans, and M. Jacob, ed., Proceedings of the 1979 Cargèse Summer Institute on Quarks and Leptons, Plenum Press, New York.

[51] J. Schechter, J.W.F. Valle. Phys. Rev., D22:2227, 1980.

[52] J. Schechter, J.W.F. Valle. Phys. Rev., D25:774, 1982.

[53] R. N. Mohapatra and G. Senjanović, Neutrino Mass and Spontaneous Parity Violation. Phys. Rev. Lett., 44:912, 1980.

[54] Z. Maki, M. Nakagawa, and S. Sakata. Prog. Theor. Phys., 28:870, 1962.

[55] R. Gandhi, C. Quigg, M. Hall Reno, I. Sarcevic. Astroparticle Physics, 5:81, 1996. 
[56] A. Cervera, A. Donini, M. B. Gavela, J. J. G. Cadenas, P. Hernandez, O. Mena, and S. Rigolin, Golden Measurements at a Neutrino Factory. Nucl. Phys., B579:17, 2000, erratum: ibid. B593, 731 (2001), hep-ph/0002108.

[57] M. Freund. Phys. Rev., D64:053003, 2001.

[58] P. Huber and W. Winter, Neutrino Factories and the 'Magic' Baseline. Phys. Rev., D68:037301, 2003, hep-ph/0301257.

[59] APS Multi-Divisional Study of the Physics of Neutrinos. http://www .aps.org/neutrino/, sponsored by the American Physical Society Divisions of: Nuclear Physics, Particles and Fields, Astrophysics, Physics of Beams, 2004.

[60] O. Mena and S. Parke, Unified Graphical Summary of Neutrino Mixing Parameters. Phys. Rev., D69:117301, 2004, hep-ph/0312131.

[61] S. Fukuda et al., Tau Neutrinos Favored over Sterile Neutrinos in Atmospheric Muon Neutrino Oscillations. Phys. Rev. Lett., 85:3999-4003, 2000, hep-ex/0009001.

[62] K. Lande and P. Wildenhain, The Homestake Chlorine Solar Neutrino Experiment: Past, Present and Future. Nucl. Phys. Proc. Suppl., 118:49-54, 2003.

[63] J. N. Abdurashitov et al., Measurement of the Solar Neutrino Capture Rate by SAGE and Implications for Neutrino Oscillations in Vacuum. Phys. Rev. Lett., 83:4686-4689, 1999, astro-ph/ 9907131 .

[64] W. Hampel et al., GALLEX Solar Neutrino Observations: Results for GALLEX IV. Phys. Lett., B447:127-133, 1999.

[65] Q. R. Ahmad et al., Measurement of the Charged Current Interactions Produced by ${ }^{8} \mathrm{~B}$ Solar Neutrinos at the Sudbury Neutrino Observatory. Phys. Rev. Lett., 87:071301, 2001, nucl-ex/ 0106015 .

[66] S. N. Ahmed et al., Measurement of the Total Active ${ }^{8}$ B Solar Neutrino Flux at the Sudbury Neutrino Observatory with Enhanced Neutral Current Sensitivity. Phys. Rev. Lett., 92:181301, 2004, nucl-ex/0309004.

[67] S. Fukuda et al., Determination of Solar Neutrino Oscillation Parameters Using 1496 Days of Super-Kamiokande-I Data. Phys. Lett., B539:179-187, 2002, hep-ex/0205075.

[68] M. Apollonio et al., Limits on Neutrino Oscillations from the CHOOZ Experiment. Phys. Lett., B466:415-430, 1999, hep-ex/9907037.

[69] F. Boehm et al., Final Results from the Palo Verde Neutrino Oscillation Experiment. Phys. Rev., D64:112001, 2001, hep-ex/0107009.

[70] K. Eguchi et al., First Results from KamLAND: Evidence for Reactor Anti-Neutrino Disappearance. Phys. Rev. Lett., 90:021802, 2003, hep-ex/0212021.

[71] E. Aliu et al., Evidence for Muon Neutrino Oscillation in an Accelerator-Based Experiment. Phys. Rev. Lett., 94:081802, 2005, hep-ex/0411038.

[72] A. Aguilar et al., Evidence for Neutrino Oscillations from the Observation of $\bar{\nu}_{e}$ Appearance in a $\bar{\nu}_{\mu}$ Beam. Phys. Rev., D64:112007, 2001, hep-ex/0104049. 
[73] E. Church et al., A Proposal for an Experiment to Measure Muon-Neutrino $\rightarrow$ ElectronNeutrino Oscillations and Muon-Neutrino Disappearance at the Fermilab Booster: BooNE, 1997, FERMILAB-PROPOSAL-0898.

[74] M.-C. Chen and K. T. Mahanthappa, Fermion Masses and Mixing and CP-Violation in SO(10) Models with Family Symmetries. Int. J. Mod. Phys., A18:5819-5888, 2003, hep-ph/0305088.

[75] G. Altarelli and F. Feruglio, Models of Neutrino Masses and Mixings, 2004, hep-ph/0405048.

[76] S. T. Petcov, On PseudoDirac Neutrinos, Neutrino Oscillations and Neutrinoless Double Beta Decay. Phys. Lett., B110:245-249, 1982.

[77] C. N. Leung and S. T. Petcov, A Comment on the Coexistence of Dirac and Majorana Massive Neutrinos. Phys. Lett., B125:461, 1983.

[78] M. Ellis, ISS Detector Working Group Report. In 8th International Workshop on Neutrino Factories, Superbeams and Betabeams (NUFACT06), UC Irvine, August 24-30,2006, http: //nufact06.physics.uci.edu/Default.aspx.

[79] P. Huber, M. Lindner, and W. Winter, Superbeams versus Neutrino Factories. Nucl. Phys., B645:3-48, 2002, hep-ph/0204352.

[80] S. Geer, Physics at an Upgraded Fermilab Proton Driver, 2005, Proceedings of the High Intensity Frontier Workshop, La Biodola, Elba, hep-ph/0507236.

[81] P. Huber, M. Lindner, M. Rolinec, T. Schwetz, and W. Winter, Prospects of Accelerator and Reactor Neutrino Oscillation Experiments for the Coming Ten Years. Phys. Rev., D70:073014, 2004, hep-ph/0403068.

[82] S. Geer. Comments Nucl. Part. Phys., A2:284-308, 2002, hep-ph/0008155.

[83] V. Barger, S. Geer, R. Raja, and K. Whisnant. Phys. Rev., D63:033002, 2000.

[84] V. Barger, S. Geer, R. Raja, and K. Whisnant. Phys.Rev., D63:113011, 2000.

[85] H. Minakata and H. Nunokawa, Exploring Neutrino Mixing with Low Energy Superbeams. JHEP, 10:001, 2001, hep-ph/0108085.

[86] G. L. Fogli and E. Lisi, Tests of Three-Flavor Mixing in Long-Baseline Neutrino Oscillation Experiments. Phys. Rev., D54:3667, 1996, hep-ph/9604415.

[87] W. Winter, Understanding CP Phase-Dependent Measurements at Neutrino Superbeams in Terms of Bi-Rate Graphs. Phys. Rev., D70:033006, 2004, hep-ph/0310307.

[88] J. Burguet-Castell, M. B. Gavela, J. J. Gomez-Cadenas, P. Hernandez, and O. Mena, On the Measurement of Leptonic CP Violation. Nucl. Phys., B608:301, 2001, hep-ph/0103258.

[89] V. Barger, D. Marfatia, and K. Whisnant, Breaking Eight-Fold Degeneracies in Neutrino CP Violation, Mixing, and Mass Hierarchy. Phys. Rev., D65:073023, 2002, hep-ph/0112119.

[90] J. Burguet-Castell, M. B. Gavela, J. J. Gomez-Cadenas, P. Hernandez, and O. Mena, Superbeams Plus Neutrino Factory: The Golden Path to Leptonic CP Violation. Nucl. Phys., B646:301, 2002, hep-ph/0207080. 
[91] A. Donini, D. Meloni, and P. Migliozzi, The Silver Channel at the Neutrino Factory. Nucl. Phys., B646:321, 2002, hep-ph/0206034.

[92] P. Huber, M. Lindner, and W. Winter. Nucl. Phys., B645:3, 2002, hep-ph/0204352.

[93] A. Donini, D. Meloni, and F. Migliozzi. J. Phys., G29:1865, 2003, hep-ph/0209240.

[94] D. Autiero et al. Eur. Phys. J., C33:243, 2004, hep-ph/0305185.

[95] A. Bueno, M. Campanelli, and A. Rubbia. Nucl. Phys., B589:577, 2000, hep-ph/0005007.

[96] P. Huber, M. Lindner, and W. Winter, GLoBES (General Long Baseline Experiment Simulator), http://www.ph.tum.de/ globes.

[97] M. Maltoni, T. Schwetz and J.W.F. Valle. Phys. Rev., D67:093003, 2003.

[98] T. Ohlsson and W. Winter, The Role of Matter Density Uncertainties in the Analysis of Future Neutrino Factory Experiments. Phys. Rev., D68:073007, 2003, hep-ph/0307178.

[99] http://protondriver.fnal.gov/.

[100] D. Neuffer, Exploration of the High-Frequency Buncher Concept. MUC-NOTE-269, 2003, http: //www-mucool.fnal.gov/mcnotes/public/pdf/muc0269/muc0269.pdf.

[101] D. Neuffer, Beam Dynamics Problems of the Muon Collaboration: $\nu$-Factory and $\mu^{+}-\mu^{-}$Colliders. MUC-NOTE-266, 2003, http://www-mucool.fnal.gov/mcnotes/public/pdf/muc0266/ muc0266.pdf.

[102] D. Neuffer, High-Frequency Buncher and Phase Rotation for the Muon Source. MUC-NOTE-181, 2000, http://www-mucool.fnal.gov/mcnotes/public/pdf/muc0181/muc0181.pdf.

[103] D. Neuffer and A. Van Ginneken. Proceedings of the 2001 Particle Accelerator Conference, 2001, http://accelconf.web.cern.ch/Accel/Conf/p01/PAPERS/TPPH162.pdf.

[104] A. Van Ginneken, Adiabatic and Vernier Bunching in Decay Channel. Technical report, Fermilab, 2001, MUC-NOTE-220, http://www-mucool.fnal.gov/mcnotes/public/ps/muc0220/ muc0220.ps.gz.

[105] R. Fernow, ICOOL: A Simulation Code for Ionization Cooling of Muon Meams. In A. Luccio and W. MacKay, eds., Proceedings of the 1999 Particle Accelerator Conference, (p. 3020), 1999, latest version available at http://pubweb.bnl.gov/people/fernow/icool/readme.html.

[106] K. McDonald, The Primary Target Facility for a Neutrino Factory Based on Muon Beams. In Proceedings of the 2001 Particle Accelerator Conference, (p. 1583), 2001, also, H.G. Kirk et al., ibid. p. 1535 and Chapter 3 in [21].

[107] A. Chao and M. Tigner, eds., Handbook of Accelerator Physics and Engineering, chapter 2 and 6, (pp. 133, 380). World Scientific, 1999.

[108] R. Geng et al., First RF Test at 4.2K of a 200MHz Superconducting Nb-Cu Cavity. In J. Chew, P. Lucas, and S. Webber, eds., Proceedings of the 2003 Particle Accelerator Conference, (p. 1309), 2003. 
[109] J. Berg, C. Johnstone, and D. Summers, Dogbone Geometry for Recirculating Accelerators. In P. Lucas and S. Webber, eds., Proceedings of the 2001 Particle Accelerator Conference, (p. 3323), 2001, D.J. Summers, Snowmass 2001, hep-ex/0208010.

[110] Vector Fields Inc., computer program OPERA-3D.

[111] S. Caspi and R. Hafalia, A Combined Function Superconducting Magnet for Fixed-Field Muon Acceleration in an Alternating Gradient Ring: First-Cut. LBNL Report SC-MAG-839, 2004.

[112] C. Johnstone and A. A. Garren, Racetrack Shaped Muon Storage Rings for a Neutrino Factory. In Proceedings of the 2001 Particle Accelerator Conference, (p. 3362), 2001, http://accelconf . web.cern.ch/accelconf/p01/PAPERS/RPPH059.PDF.

[113] C. Johnstone and F. Méot, On Fringe Field Effects in the Fermilab 50 GeV Muon Storage Ring. In P. Lucas and S. Webber, eds., Proceedings of the 2001 Particle Accelerator Conference, (p. 3359), 2001.

[114] K. Makino and M. Berz, COSY INFINITY version 8. Nucl. Instrum. \& Meth., A427:338, 1999.

[115] F. Lemuet, F. Méot, and G. Rees, 6-D Beam Dynamics in an Isochronous FFAG Ring. In Proceedings of the 2005 Particle Accelerator Conference, (p. 2693), 2005, http://accelconf .web. cern.ch/AccelConf/p05/PAPERS/RPPT067.PDF.

[116] E. Forest et al., Polymorphic Tracking Code. KEK report 2002-3; also CERN-SL-2002-044(AP), 2002.

[117] N. Mokhov, C. Johnstone, and B. L. Parker, Beam-Induced Energy Deposition in Muon Storage Rings. In P. Lucas and S. Webber, eds., Proceedings of the 2001 Particle Accelerator Conference, (p. 3401), 2001.

[118] J. R. J. Bennett et al. http://doc.cern.ch//archive/electronic/cern/preprints/intc/ public/intc-2004-016.pdf.

[119] H. G. Kirk et al., A High Power Target Experiment. In Proceedings of the 2005 Particle Accelerator Conference, (p. 3745), 2005, http://accelconf.web.cern.ch/AccelConf/p05/PAPERS/ RPPT067.PDF.

[120] R. Edgecock, The MICE Experiment. J. Phys., G29:1601, 2003, see also the MICE Proposal, http://mice.iit.edu/mnp/MICE0021.pdf .

[121] http://www-kuno.phys.sci.osaka-u.ac.jp/ yoshida/ISS/index.html.

[122] http://www.fnal.gov/projects/muon_collider/cool/cool.html.

[123] S. A. Bogacz, Low Energy Stages-Dogbone Muon RLA. In M. Aoki, Y. Iwashita, and M. Kuze, eds., Proc. Sixth International Conf. on Neutrino Factories and Superbeams, Nucl. Phys. B (Proc. Suppl.) 149, (p. 309), 2005.

[124] D.J. Summers, J.S Berg, A.A. Garren, R.B. Palmer. J. Phys., G29:1727, 2003.

[125] Y. Yonemura et al., Development of FFAG Accelerator at KEK. In Proceedings of the 2005 Particle Accelerator Conference, (p. 1943), 2005, http://accelconf.web.cern.ch/AccelConf/ p05/PAPERS/FPAE026.PDF. 
[126] A. Hassenein et al., An R\&D Program for Targetry and Capture at a Neutrino Factory and Muon Collider Source. Nucl. Instrum. ES Meth., A503:70, 2003.

[127] J. R. Haines and C. C. Tsai, Graphite Sublimation Tests for the Muon Collider/Neutrino Factory Target Development Program. Technical report, ORNL, 2002, ORNL/TM-2002/27.

[128] C. C. Tsai, T. A. Gabriel, J. R. Haines, and D. A. Rasmussen, Graphite Sublimation Tests for the Muon Collider/Neutrino Factory Target Development Program. In Proceedings of the 21st IEEE/NPSS Symposium on Fusion Engineering, 2005.

[129] N. Simos et al., Target Material Irradiation Studies for High-Intensity Accelerator Beams. In M. Aoki, Y. Iwashita, and M. Kuze, eds., Proc. Sixth International Conf. on Neutrino Factories and Superbeams, Nucl. Phys. B (Proc. Suppl.) 149, (p. 259), 2005.

[130] J. R. J. Bennett, UK Studies of Solid Targets for Neutrino Factories. In M. Aoki, Y. Iwashita, and M. Kuze, eds., Proc. Sixth International Conf. on Neutrino Factories and Superbeams, Nucl. Phys. B (Proc. Suppl.) 149, (p. 262), 2005.

[131] A. Fabich, High-Power Proton Beam Shocks and Magnetohydrodynamics in a Mercury Jet Target for a Neutrino Factory. Ph.D. thesis, Technische Universität Wien, Austria, November 2002, http://doc.cern.ch/archive/electronic/cern/preprints/thesis/thesis-2002-038.pdf.

[132] R. Samulyak, Numerical Simulation of Hydro- and Magnetohydro-dynamic processes in the Muon Collider Target. Lecture Notes in Computer Science, 2331:391, 2002.

[133] K. Yoshimura, Y. Yamanoi, P. Sievers, and B. Autin, Target Material Irradiation Studies for High-Intensity Accelerator Beams. In M. Aoki, Y. Iwashita, and M. Kuze, eds., Proc. Sixth International Conf. on Neutrino Factories and Superbeams, Nucl. Phys. B (Proc. Suppl.) 149, (p. 265), 2005.

[134] J. Norem, et al. Phys. Rev. ST Accel. Beams, 6:072001, 2003, also MUC-NOTE-226 (2001).

[135] R. A. Rimmer et al., Fabrication of the Prototype $201.25 \mathrm{MHz}$ Cavity for a Muon Ionization Cooling Experiment. In Proceedings of the 2005 Particle Accelerator Conference, (p. 2080), 2005, see http://accelconf .web.cern.ch/AccelConf/p05/PAPERS/TPPT029.PDF.

[136] K. Yonehara et al., High-Pressure RF Cavities for Muon Beam Cooling. In M. Aoki, Y. Iwashita, and M. Kuze, eds., Proc. Sixth International Conf. on Neutrino Factories and Superbeams, Nucl. Phys. B (Proc. Suppl.) 149, (p. 259), 2005.

[137] M.A. Cummings et al., Current LH $\mathrm{LH}_{2}$ Absorber R\&D in MUCOOL. J. Phys., G29:1689, 2003.

[138] http://mice.iit.edu/.

[139] R. L. Geng et al., 200 MHz Superconducting RF Cavity Development for RLAs. In A. Para, ed., Proc. Fifth International Conf. on Neutrino Factories and Superbeams, AIP Conf. Proceedings, (p. 445), AIP Press, New York, 2004.

[140] C. Ohmori et al., Ultra-High Field Gradient RF for Bunch Rotation. In M. Aoki, Y. Iwashita, and M. Kuze, eds., Proc. Sixth International Conf. on Neutrino Factories and Superbeams, Nucl. Phys. B (Proc. Suppl.) 149, (p. 280), 2005. 
[141] E. Keil and A. Sessler, An Electron Model of an FFAG Muon Accelerator. Technical report, CERN, 2003, CERN-NUFACT-Note-135, http://doc.cern.ch/archive/electronic/ cern/preprints/ab/ab-2003-095.pdf.

[142] C. Johnstone, Hardware Development for EMMA. In G. D. Lellis, P. Migliozzi, A. Paoloni, and F. Terranova, eds., Proc. Seventh International Conf. on Neutrino Factories and Superbeams, Nucl. Phys. B (Proc. Suppl.), to be published.

[143] M.S. Zisman, Accelerator Working Group Summary Report. In 8th International Workshop on Neutrino Factories, Superbeams and Betabeams (NUFACT06), UC Irvine, August 24-30,2006, http://nufact06.physics.uci.edu/Workshop/Slides/Accel-SummaryTalk-8-24-06-R2. ppt. 